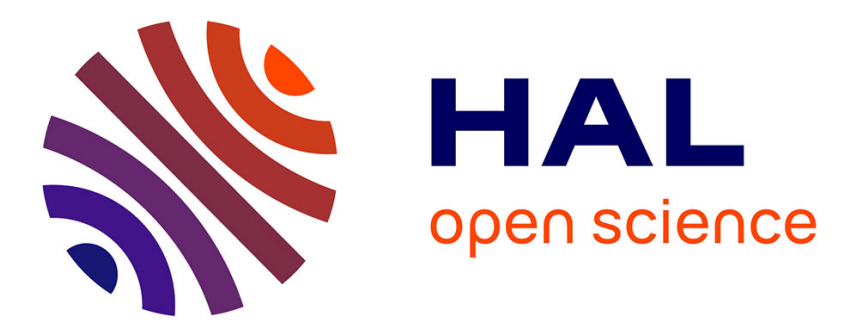

\title{
Metasurfaces for biomedical applications: imaging and sensing from a nanophotonics perspective
}

Shuyan Zhang, Chi Lok Wong, Shuwen Zeng, Renzhe Bi, Kolvyn Tai, Kishan Dholakia, Malini Olivo

\section{- To cite this version:}

Shuyan Zhang, Chi Lok Wong, Shuwen Zeng, Renzhe Bi, Kolvyn Tai, et al.. Metasurfaces for biomedical applications: imaging and sensing from a nanophotonics perspective. Nanophotonics, 2020, 10 (1), pp.259 - 293. 10.1515/nanoph-2020-0373 . hal-03123677

\section{HAL Id: hal-03123677 https://hal.science/hal-03123677}

Submitted on 28 Jan 2021

HAL is a multi-disciplinary open access archive for the deposit and dissemination of scientific research documents, whether they are published or not. The documents may come from teaching and research institutions in France or abroad, or from public or private research centers.
L'archive ouverte pluridisciplinaire HAL, est destinée au dépôt et à la diffusion de documents scientifiques de niveau recherche, publiés ou non, émanant des établissements d'enseignement et de recherche français ou étrangers, des laboratoires publics ou privés. 


\section{Review}

\section{Shuyan Zhang, Chi Lok Wong, Shuwen Zeng, Renzhe Bi, Kolvyn Tai, Kishan Dholakia} and Malini Olivo*

\section{Metasurfaces for biomedical applications: imaging and sensing from a nanophotonics perspective}

https://doi.org/10.1515/nanoph-2020-0373

Received July 4, 2020; accepted August 17, 2020; published online September 7, 2020

\begin{abstract}
Metasurface is a recently developed nanophotonics concept to manipulate the properties of light by replacing conventional bulky optical components with ultrathin (more than $10^{4}$ times thinner) flat optical components. Since the first demonstration of metasurfaces in 2011, they have attracted tremendous interest in the consumer optics and electronics industries. Recently, metasurface-empowered novel bioimaging and biosensing tools have emerged and been reported. Given the recent advances in metasurfaces in biomedical engineering, this review article covers the state of the art for this technology and provides a comprehensive interdisciplinary perspective on this field. The topics that we have covered include metasurfaces for chiral imaging, endoscopic optical coherence tomography, fluorescent imaging, superresolution imaging, magnetic resonance imaging, quantitative phase imaging, sensing of antibodies, proteins, DNAs, cells, and cancer biomarkers. Future directions are discussed in twofold: application-specific biomedical metasurfaces and bioinspired metasurface devices. Perspectives on challenges and opportunities of metasurfaces, biophotonics, and translational biomedical devices are also provided. The objective of this review article is to inform and stimulate interdisciplinary research: firstly, by
\end{abstract}

*Corresponding author: Malini Olivo, Laboratory of Bio-Optical Imaging, Singapore Bioimaging Consortium, A*STAR, Singapore, Singapore, E-mail: malini_olivo@sbic.a-star.edu.sg Shuyan Zhang, Chi Lok Wong, Renzhe Bi and Kolvyn Tai, Laboratory of Bio-Optical Imaging, Singapore Bioimaging Consortium, $A *$ STAR, Singapore, Singapore. https://orcid.org/0000-0002-1286-4856 (S. Zhang). https://orcid.org/0000-0001-7173-064X (R. Bi) Shuwen Zeng, XLIM Research Institute, UMR CNRS, Paris, France. https://orcid.org/0000-0003-2188-7213

Kishan Dholakia, SUPA, School of Physics \& Astronomy, University of St Andrews, St Andrews, UK; and Department of Physics, Yonsei University, Seoul, South Korea introducing the metasurface concept to the biomedical community; and secondly by assisting the metasurface community to understand the needs and realize the opportunities in the medical fields. In addition, this article provides two knowledge boxes describing the design process of a metasurface lens and the performance matrix of a biosensor, which serve as a "crash-course" introduction to those new to both fields.

Keywords: bioimaging; biophotonics; biosensing; metasurface; nanophotonics.

\section{Introduction}

From auroras and rainbows to the human eye - light has fascinated scientists for centuries. Today, optical technologies - from lasers to solar cells to cameras - harness light to advance physics and serve societal needs. The understanding and use of the fundamental properties of light are important parts of the progress of human history. Recently, the development of photonic materials, circuitry, devices, and probes on the nanoscale has opened up new opportunities for controlling light in the subwavelength regime.

In 1968, Veselago theoretically predicted the generation of artificial materials [1] by engineering their permittivity and permeability. This was eventually realized by the studies of Pendry et al. [2] and Smith et al. [3] that helped realize Veselago's prediction and have revolutionized our approach to electromagnetics. Such so-termed metamaterials are artificial composite nanostructures that possess unique properties for controlling light and demonstrate numerous exciting new optical effects and applications that cannot be achieved by natural materials [4-7]. Metasurfaces are structures that benefit from the reduced dimensionality of metamaterials. They are relatively easy to fabricate and possess a smaller footprint (thickness of less than a millimeter) than conventional optical components. They consist of optical components (or meta-atoms) patterned on a surface with subwavelength dimensions, 
which shape the wavefront of light to introduce a desired spatial profile of the optical phase. Since their discovery [8], their exceptional optical properties have led to the development of ultrathin optical devices with various functionalities outperforming their conventional bulky counterparts and also demonstrating new optical phenomena covering a wide range of electromagnetic spectrum from the visible to the terahertz $(\mathrm{THz})$ region. Such metasurface-based flat devices represent a new class of optical components that are compact, flat, and lightweight. Numerous devices based on metasurfaces have been developed, including metasurface lenses (metalenses) [9-19], waveplates [20-23], polarimeters [24-26], and holograms [27-29]. For metalenses, in particular, numerical aperture (NA) as high as 0.97 was demonstrated [15]. Metalenses with NA $>1$ was realized by immersion metalenses [30]. There are review papers dedicated to the fundamentals, progress, and applications of metasurfaces. Some representative examples can be found in the references [31-51].

Metasurfaces have generated tremendous interest in the consumer optical and electronics industries. In addition, metasurface-empowered novel bioimaging and biosensing devices have also emerged and reported recently. For many optically based bioimaging devices, their bulk footprint and heavy physical weight have limited their usage in clinical settings. One of the bottlenecks is the large footprint of conventional optical components, such as lens assemblies and spectrometers. There are also limitations in the performance of optical components, for example, spherical lenses suffer from spherical aberrations which greatly impact the imaging quality and require often additional corrective optics. Besides imaging, sensing is another important research field in biomedical engineering. There has been a crucial need for a rapid and reliable sensing method for microorganisms such as bacteria and viruses. Current methods are tedious and time-consuming, as the growth of these micro-organisms can take up to days and weeks. While methods such as polymerase chain reaction (PCR) and mass spectrophotometry have managed to reduce the time required for the measurements, the steps involved are exceptionally intricate making it challenging to be used for swift detection. As for cancer detection, current detection methods are pricy and time-consuming. Metasurface biosensors can provide a solution for rapid, label-free detection of various micro-organisms and cancer cells.

Given the recent advances and progress of metasurfaces in biomedical engineering, reviews published on the merging of these research areas are gaining attention [52-54]. However, their scopes are limited to either bioimaging or biosensing application. This review article aims to cover the state-of-the-art technology development for metasurfaces and a comprehensive and detailed account of both biomedical applications. The objective of this review is to stimulate interdisciplinary research by introducing the metasurface concept to the biomedical community and informing the metasurface community about the needs and opportunities in the biomedical research area.

Figure 1 gives an overview of the structure of this review paper. Firstly, the general concepts of metasurfaces will be introduced including the governing principles and fabrication techniques. Secondly, recent advances in metasurfaces for various bioapplications will be reviewed. This includes optical chiral imaging, endoscopic optical coherence tomography (OCT), fluorescent imaging, super-resolution imaging, magnetic resonance imaging (MRI), and quantitative phase imaging (QPI). Thirdly, applications of metasurface in biosensing are discussed, including the detection of antibodies and proteins, DNAs, cells, and cancer biomarkers. Lastly, future directions are described including various metasurface functions demonstrated for non-biomedical applications that could be potentially useful for biomedical applications and the development of biomimetic metasurface devices. We conclude with perspectives on present challenges to make metasurface-based biomedical devices toward commercialization and for translational research.

\section{Fundamentals of metasurfaces}

In contrast to conventional optical components that achieve wavefront engineering by phase accumulation, metasurfaces control the wavefront of light using arrays of optical resonators with subwavelength dimensions. By tailoring the optical properties of each meta-atom, one can spatially control the phase, amplitude, and polarization of the light and consequently shape the wavefront. There has been an increasing interest in the past decade in the field of metasurface as it promises remarkable capabilities for light molding beyond that offered by conventional planar interfaces. Several variations of metasurfaces have arisen to improve their performance, changes such as the transition from plasmonic materials to the use of dielectric materials to improve the efficiency and using different meta-atoms for additional optical functionalities. The fundamentals of metasurfaces are reviewed below from the physical concept, the design methodology, to the fabrication techniques.

\subsection{Governing equations}

When an electromagnetic wave hits the boundary between two media with different refractive indices, the wave splits 

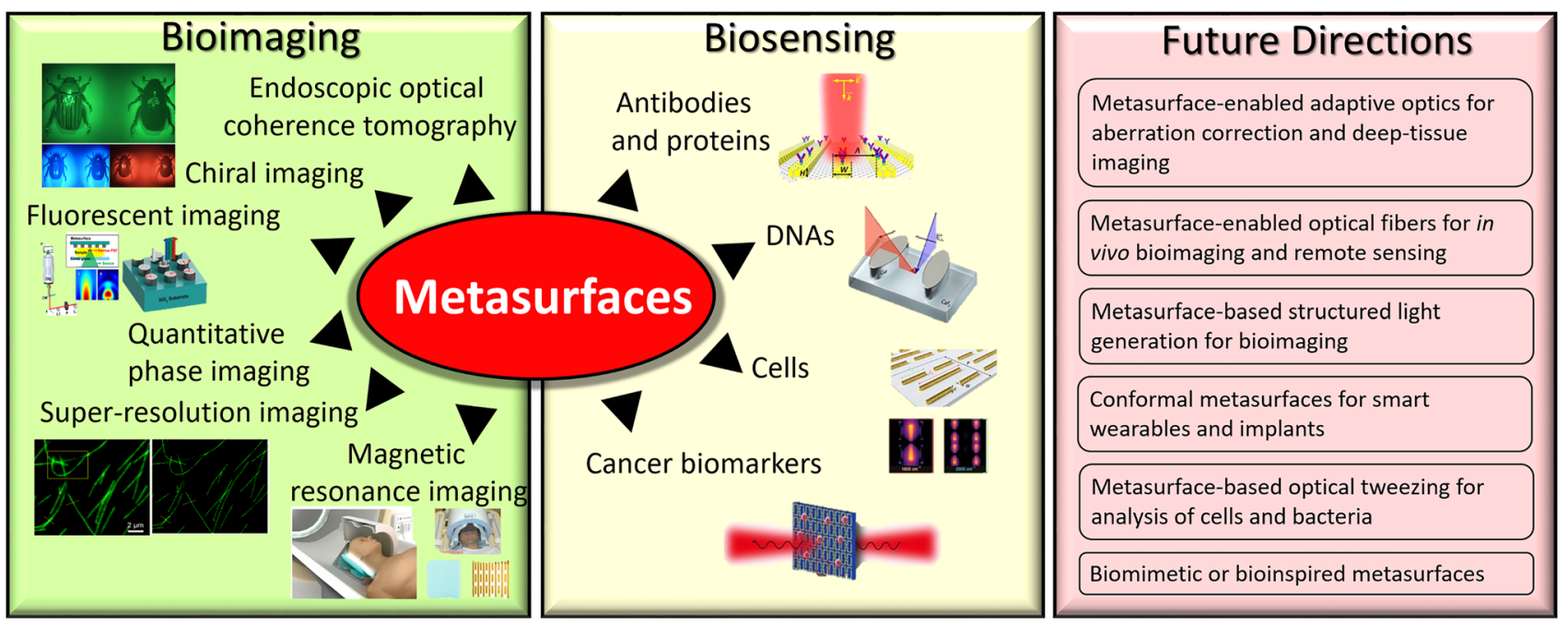

Figure 1: Overview of metasurfaces for bioimaging and biosensing applications and future directions. Chiral imaging: Reproduced with permission from Khorasaninejad et al., Nano Lett. 16, 4595-4600 (2016) [96]. Copyright 2016 American Chemical Society. Direct link: https:// pubs.acs.org/doi/abs/10.1021/acs.nanolett.6b01897. Further permissions related to the material excerpted should be directed to the ACS. Fluorescent imaging: Adapted from Lee et al., Opt. Mater. Express 9, 3248 (2019) [99]. Copyright 2019 Optical Society of America. Superresolution imaging: Reproduced with permission from Bezryadina et al., ACS Nano 12, 8248-8254 (2018) [109]. Copyright 2018 American Chemical Society. Magnetic resonance imaging: Adapted from Schmidt et al., Sci. Rep. 7, 1678 (2017) [113]. Licensed under Creative Commons Attribution 4.0 International License. Antibodies and proteins: Reproduced with permission from Guo et al., ACS Photonics 1, 221-227 (2014) [135]. Copyright 2014 American Chemical Society. DNAs: Adapted from Leitis et al., Sci. Adv. 5, eaaw2871 (2019) [138]. Distributed under a Creative Commons Attribution NonCommercial License 4.0 (CC BY-NC). Cells: Reproduced with permission from Rodrigo et al., Nat. Commun. 9, 2160 (2018) [145]. Licensed under a Creative Commons Attribution 4.0 International License. Cancer biomarkers: Reproduced with permission from Yan et al., Biosens. Bioelectron. 126, 485-492 (2019) [148]. Copyright 2019 Elsevier.

into two different waves, the reflected wave is rebounded back into the first medium and the transmitted wave enters the second medium. The angle at which these waves travel is determined by Snell's law. However, Snell's law does not take into account the abrupt phase discontinuities introduced by the metasurface.

The governing equations of the interaction of light and the metasurface for transmission (Eq. 1) and reflection (Eq. 2) are summarized as the generalized Snell's laws in threedimensional (3D) derived from Fermat's principle $[8,55,56]$ :

$$
\begin{aligned}
& \left\{\begin{array}{c}
n_{t} \sin \left(\theta_{t}\right)-n_{i} \sin \left(\theta_{i}\right)=\frac{\lambda}{2 \pi} \frac{d \phi}{d x} \\
\cos \left(\theta_{t}\right) \sin \left(\varphi_{t}\right)=\frac{1}{n_{t}} \frac{\lambda}{2 \pi} \frac{d \phi}{d y}
\end{array}\right. \\
& \left\{\begin{array}{c}
\sin \left(\theta_{r}\right)-\sin \left(\theta_{i}\right)=\frac{1}{n_{i}} \frac{\lambda}{2 \pi} \frac{d \phi}{d x} \\
\cos \left(\theta_{r}\right) \sin \left(\varphi_{r}\right)=\frac{1}{n_{r}} \frac{\lambda}{2 \pi} \frac{d \phi}{d y}
\end{array}\right.
\end{aligned}
$$

where the $x-z$ plane is the plane of incidence and the metasurface is located on the $x-y$ plane. $n_{t}, n_{i}$ and $n_{r}$ are the refractive index of the mediums, $\theta_{\mathrm{t}}, \theta_{\mathrm{r}}$, and $\theta_{\mathrm{i}}$ represent the angle of transmission, reflection, and incidence in the $x-z$ plane, respectively. $\varphi_{\mathrm{t}}$ and $\varphi_{\mathrm{r}}$ represent the angle of transmission and reflection in the $y-z$ plane, respectively. $\frac{d \phi}{d x}$ and $\frac{d \phi}{d y}$ is the phase gradient parallel and perpendicular to the plane of incidence, respectively. Note that the lack of a phase gradient will result in a normal form of Snell's law. These equations imply that reflected and transmitted waves can be arbitrarily bent in their respective half space, by engineering the phase gradient's direction and magnitude.

\subsection{Design principles}

The building blocks of metasurfaces are the meta-atoms on a subwavelength scale. These meta-atoms can be of different materials, such as plasmonic materials, dielectric materials, or a combination of the two. Typical plasmonic materials include metals, transparent conducting oxides (e.g. indium tin oxide), and two-dimensional (2D) materials. Strong light-matter interactions occur in these materials through plasmonic resonances which originate from a process called polarization: the dynamic response of electrons inside the material to the external electromagnetic field. The resonant wavelength depends on the sizes, shapes, materials, and surrounding media of the meta- 
atoms [31, 33]. In general, a phase shift of $\pi$ is generated with the resonance process, but a phase shift of $2 \pi$ is required to fully manipulate the properties of light. One of the earlier demonstrations to achieve a phase shift of $2 \pi$ for a single-mode dipolar resonance is v-shaped antennas, where a symmetric mode and an antisymmetric mode can be supported at the same time [8]. Over the years, researchers have used different shapes of plasmonic metaatoms including 1D nanostructures (grooves, gratings, slits or ribbons), 2D nanostructures (holes, bow-tie shaped antennas, v-shaped antennas, split-ring resonators), and colloidal nanocrystals on solid substrates or in optical films [57]. Although plasmonic materials can provide strong optical resonances, one of the major limitations is the ohmic loss (resistive loss), where the energy of the incident light is transferred to heat in the material. For applications that require high efficiency of the device, this is nonideal because the ohmic loss results associated with heat dissipation in the plasmonic materials represent a reduction of the overall efficiency.

To overcome this limitation on reduced efficiency, a class of metasurface devices based on dielectric materials were reported. Representative review papers on this research field can be found in $[50,51]$. Typical dielectric materials used include titanium dioxide, silicon, germanium, and tellurium. The real parts of the refractive index of these materials are high so that they can manipulate the properties of light through Mie resonance [58] and achieve a phase shift of $2 \pi$ with an enhancement of both electric and magnetic fields. The imaginary part of the refractive index is almost negligible so that there is minimal loss due to resistive heating and hence the overall device efficiency remains high.

Besides the type of materials chosen for the metaatoms, the efficiency of a metasurface device in controlling the transmitted light is also dependent on the reflection at the light-matter interface, i.e. to boost the efficiency for transmission, complete elimination of reflection is essential. This can be achieved by matching the impedance of a metasurface with that of the free space [59]. For example, Huygen's metasurfaces may comprise nonperiodic nanostructures or multilayered structures to engineer the surface impedance locally [46]. According to the HuygenFresnel principle $[60,61]$, every point on a wavefront is itself the source of spherical wavelets and propagates with the same speed as the source wave. The secondary wavelets emanating from different points interfere and the sum of these wavelets forms the new wavefront which is represented as the line tangent to the wavelets. For a Huygen's metasurface, secondary waves transmitted through or reflected from the metasurface gain different phase shifts from the light interaction with the meta-atoms, and they can interfere. Therefore, by carefully designing the optical response of the meta-atoms, one can generate an arbitrary wavefront at will.

In addition to engineering the resonances of metaatoms, there is another metasurface design approach based on the Pancharatnam-Berry (P-B) phase [62, 63], namely the geometric phase, to achieve a phase shift of $2 \pi$. For metasurface designs based on $\mathrm{P}-\mathrm{B}$ phase, the amount of phase shift of generated by a meta-atom is $\pm 2 \theta(x, y)$, where $\theta$ is the orientation angle of the meta-atom, and the sign of the phase shift (i.e. the addition or subtraction of phase) is determined by the handedness of the incident polarized light (left circularly polarized [LCP] or right circularly polarized [RCP] light). When the meta-atom is arranged to rotate at an angle from 0 to $\pi$, the phase shift can be continuously tuned from 0 to $2 \pi$. Since the phase shift is determined by the geometry, $\mathrm{P}-\mathrm{B}$ phase metasurfaces typically exhibit ultrabroadband performance, however, they only operate on circularly polarized incident light.

So far, we have discussed the overall building mechanisms of a metasurface for the manipulation of light properties. To add further functionalities, recently, a variety of tuning mechanisms have been proposed [41]. For instance, flexible substrates were used for conformal metasurfaces, mechanically, and electrically tunable metasurfaces [4, 6466]. The high flexibility and stretchability of soft materials such as polydimethylsiloxane (PDMS) and poly(methyl methacrylate) (PMMA) have made it possible for the substrates to be wrapped and bent $[67,68]$. In this way, the spacing between meta-atoms is changed and hence the properties of the metasurface become tunable. Phase transition materials have been widely used in optical datastorage systems and nanophotonic systems based on their tunable optical properties. Commonly used phase change materials include vanadium dioxide $\left(\mathrm{VO}_{2}\right)$ [69-72] and GeSbTe alloys [73], which show a dramatic change in optical properties when undergoing phase transitions triggered by thermal energy or chemical doping. For example, the change in the refractive index of $\mathrm{VO}_{2}$ in the mid-infrared wavelength region can reach $\Delta \boldsymbol{n} \approx 4$ and $\Delta \boldsymbol{k} \approx 8$ [74]. This translates to a change in the properties of the metasurface made of these materials. Liquid crystals have also been extensively used for the dynamic control of the optical properties because of their broadband optical nonlinearity and birefringence. Their change of refractive index can be externally tuned by temperature, light, and electric or magnetic fields $[75,76]$. Microfluidic technology has been applied to tunable metalenses by controlling the air pressure of the pneumatic valves to change the distribution of liquid metals within a unit cell [77]. In addition, two-dimensional materials with extraordinary optical and electrical properties such as graphene were 
integrated into electrically tunable metasurfaces. Their optical properties were made tunable through a change in the charge carrier density which was controlled by the gate voltage $[78,79]$. For example, in [79], a gate voltage change of $80 \mathrm{~V}$ resulted in a modulation depth of up to $100 \%$ and a wavelength shift of $1.5 \mu \mathrm{m}$ in the mid-infrared region.

A brief summary of the design steps of a metalens is given in Box 1 with simulation illustrations. A more general flow chart of designing a general metasurface device not only a lens can be found in [44].

\subsection{Fabrication techniques}

The fabrication techniques of metasurfaces are microfabrication and nanofabrication techniques depending on the feature size of the metasurface which typically depending on the operating wavelength. For applications with a shorter wavelength range such as in the visible wavelength range, a small feature size is required, so nanofabrication techniques such as electron-beam lithography, focused ion beam (FIB) lithography, and

\section{Box 1 Designing a metalens}

\section{Step 1: Choice of unit cell}

Metasurfaces consist of carefully arranged "unit cells" or "meta-atoms" with subwavelength structures. The optical response (phase, amplitude, and polarization) of the unit cell to the incident wave changes with its geometry (height, length, width, shape, material, etc.). For a transmission-based metalens, a simple unit cell could be a periodic 2D array of nanopillars. It is the simplest design and the optical response is independent of the incident polarization.

\section{Step 2: Generation of phase map}

Normally, the incident wavelength of a metasurface device is known, i.e., it is not a variable. The variables are geometric parameters of the unit cell, e.g. height, radius, and period. By scanning these parameters, one can generate a lookup table of the phase, i.e., a phase map. The height and period that give desired transmission (uniform and high transmission values) and phase (phase coverage from 0 to $2 \pi$ ) properties is chosen. Then the phase of the nanopillar array can be controlled by changing its radius. The phase map describes the relationship between the phase and the radius of the nanopillar for a specific height and period.

\section{Step 3: Mapping of phase profile}

With the knowledge of the phase map, it is possible to create a metasurface with an arbitrary phase profile. Note that a phase profile is different from the phase map described in Step 2. A phase profile is the target phase of a metasurface device as a function of the spatial position, i.e., placement of unit cells with different radii based on the phase map. For the case of a metalens, the phase profile is a hyperbola (for $2 \mathrm{D}$ focusing into a focal line): $\varphi(x)=-2 \pi / \lambda \times\left(\sqrt{f^{2}}+x^{2}-f\right.$ ) or a hyperboloid (for 3D focusing into a focal point): $\varphi(x, y)=-2 \pi / \lambda \times\left(\sqrt{f^{2}}+x^{2}+y^{2}-f\right)$, where $\lambda$ is the wavelength, $f$ is the focal length of the metalens, $x, y$ are the spatial coordinates. At a given spatial position, it is possible to calculate the required nanopillar radius that can produce a specific phase value (closest to the target phase value) based on the phase map.

Note that for a large metalens (lens diameter $>\mathrm{mm}$ ) that may consist of billions of such nanopillars, the file size that stores the radius and position information is huge beyond the fabrication capability. In such cases, radial symmetry with fixed edge-to-edge distance or other non-periodic arrangement (i.e. variable period) of the nanopillars can be utilized to reduce the file size.

\section{Step 4: Modeling of full lens}

The full metalens is constructed based on the phase map data in Step 2 and the target phase profile in Step 3. The metalens is modeled using finite-difference time-domain (FDTD) technique. Commercial FDTD software is available. The incident waves pass through the metalens when propagating and eventually interfere constructively at the designed focal length. By studying the focal profile (intensity vs position), it is possible to determine the full width at half maximum (FWHM) of the focused spot size and compare it with the theoretical diffraction limit. 


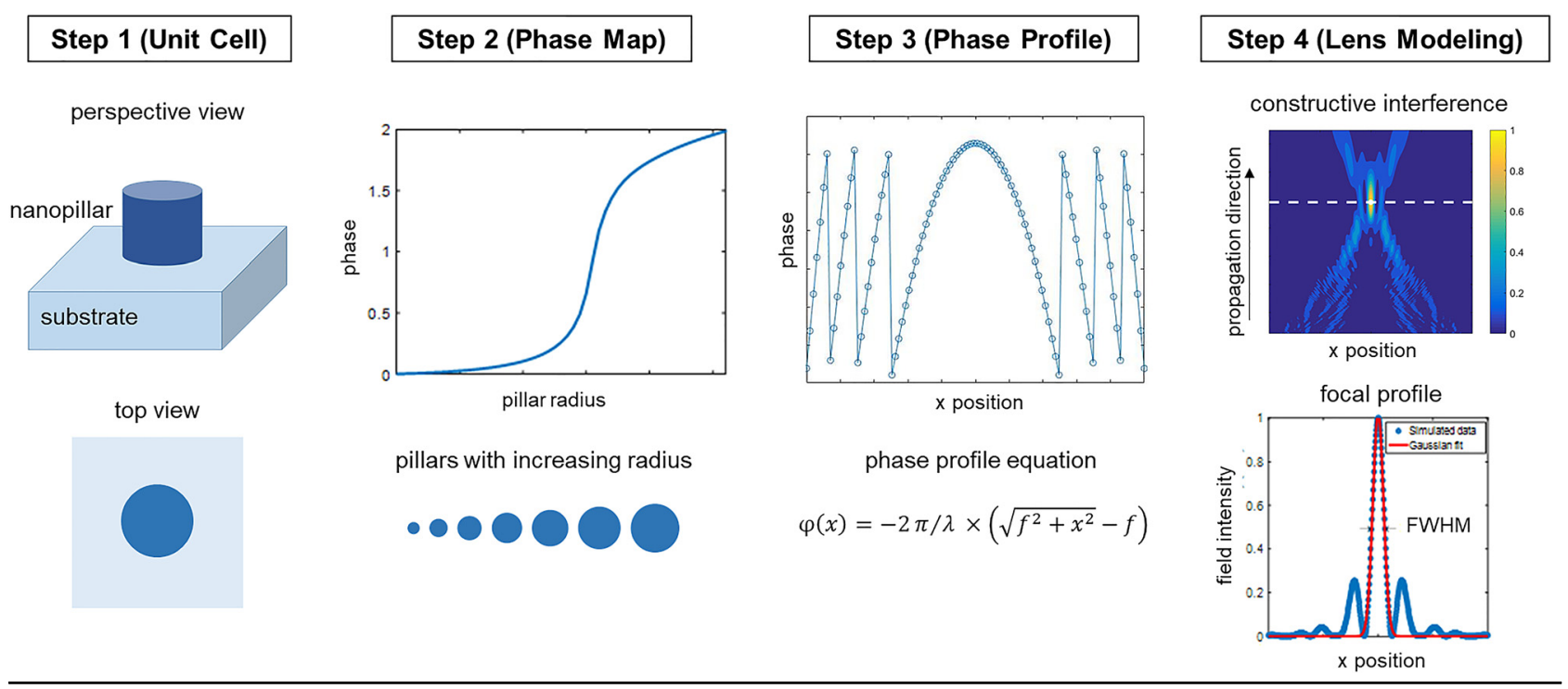

nanoimprint lithography are normally used. For applications with a longer wavelength range such as in the infrared wavelength range, the feature size requirement is relaxed, so microfabrication techniques may be sufficient, such as photolithography. These are top-down-based lithography techniques, and bottom-up-based lithography technique such as self-assembly lithography has also been reported for the fabrication of metasurfaces. A detailed review of various fabrication techniques including the advantages and disadvantages can be found in a study by $\mathrm{Su}$ et al. [47].

Photolithography is a high-throughput fabrication method at the microscale and nanoscale. It is the most commonly used fabrication method in the semiconductor industry due to its advantages, such as high reproducibility, high yield, low cost, and mass production availability. Photolithography uses a photomask to cover the photoresist which is a light-sensitive substrate. There are two types of photoresist: positive and negative resist. The photomask is used to transfer the pattern into the photoresist after exposure to light. The photoresist is then used to deposit materials in the desired pattern or used as a photomask for etching patterns onto a substrate placed under the photoresist. After the pattern is transferred to the wafer, the photoresist is removed. The resolution of a photolithography system depends on the wavelength of the light source and the reduction lens system. Traditional UV photolithography based on $\mathrm{Hg}$ lamps has a resolution of $1 \mu \mathrm{m}$ which is suitable for metasurfaces with a large feature size at longer wavelengths (mid-infrared wavelength range and beyond). For example, in a study by Zhang et al. [9, 80, 81], metasurface devices in the mid-infrared wavelength range were fabricated using photolithography, and in a study by Chen et al. [32] and $\mathrm{Hu}$ et al. [82], metasurface devices were designed in the terahertz and microwave frequencies. Recently, there has been a trend in using deep-UV photolithography with an excimer laser source to fabricate metasurfaces at the visible [14] and the near-infrared wavelength $[10,83]$ in an attempt for scalability and mass production.

Electron beam lithography, commonly known as e-beam lithography is a form of fabrication that produces patterns with high resolution down to sub $10 \mathrm{~nm}$. It does not require a mask and uses an electron beam to etch the pattern directly onto the resist. The electron beam changes the solubility of the resist, and the resist is then selectively removed using a solvent. As with photolithography, the purpose of e-beam lithography is to etch patterns into the resist and deposit other materials in the desired pattern or for further etching. Metasurfaces fabricated using e-beam lithography are normally sub-mm [12, 15, 48]. This form of lithography has low throughput, limiting it to low volume production and research purposes only.

FIB lithography is another technique for fabricating patterns with high resolution. It uses a finely focused beam of ions (usually gallium, $\mathrm{Ga}^{+}$) operating with high beam currents for localized sputtering or milling. The sputtering process is a deposition of Ga atoms on the surface. Milling occurs by the physical bombardment of high energy Ga ions to remove the surface materials. The resolution can reach 10-15 nm. One advantage of using FIB for micromachining or nanomachining is that it is a direct writing process, i.e. no additional etching step is necessary. Metasurfaces fabricated using FIB have been reported in a study by Lin et al. [84] and Liu et al. [85]. Especially it has been widely used to pattern metasurfaces in fiber [24] or on the fiber facets [86, 87]. Compared to electron beam 
lithography, FIB is also low throughput, but the material selection of the sample is relatively limited. The milling rate varies and greatly depends on the sample material. FIB can be used to pattern nanostructures on fiber tips, but electron beam lithography cannot.

Nanoimprint lithography [88] is a high throughput method that has a high resolution, is low cost, and able to imprint a large area and can be done in a single step. Nanoimprint lithography uses a mold (fabricated using photolithography or e-beam lithography) to imprint the pattern onto a substrate coated with a UV-curable resist layer, the substrate is then processed by heat or UV light followed by a release step and etching process to remove the residual. Nanoimprint lithography is an ideal choice for low cost and large volume metasurface fabrication with a resolution down to $100 \mathrm{~nm}$ [89-91], which is sufficient enough for most metasurfaces in the visible wavelength range.

Self-assembly lithography [92], also known as directed self-assembly, is a form of fabrication that can be used to mass produce microparticles and nanoparticles for devices of the high complexity. This method can achieve spontaneous assembly of the pattern by intermolecular attraction and repulsion. Direct assembly lithography uses block copolymer which can be engineered to have different morphology. Surface interactions and thermodynamics between the block copolymers allow the formation of shapes and patterns. However, the interactions will assemble randomly; hence the block copolymer needs to be directed to form the desired pattern. This can be achieved through epitaxy, using a chemical pattern on the surface to direct, or graph epitaxy, using surface topology to align the block copolymers. Examples of metasurfaces fabricated using this technique are included in the studies by Kim et al. [93], Mayer et al. [94] and Wu et al. [95]. This form of lithography is high throughput and low cost but faces challenges in achieving the uniformity and may only generate limited patterns.

\section{Bioimaging}

Optical bioimaging usually obtains information based on the transmitted, reflected, and scattered light. The information obtained reflects the intensity, phase, and polarization properties of light. Metasurfaces can control these properties through the interaction of light with the metaatoms and hence improve the imaging quality. In this section, metasurface-based imaging modalities such as chiral imaging study the polarization of light; endoscopic OCT, fluorescent imaging, super-resolution imaging, and
MRI study the intensity of light; QPI study the phase of light. Features of metalenses for imaging include diffraction-limited focusing, high-quality imaging, and multifunctionalities [48]. As the phase profile of a metalens can be designed accurately, it can closely approximate the ideal phase of a perfect lens to focus light at the theoretical fundamental diffraction limit without spherical aberrations. The nanostructure arrangement to realize the designed phase profile also gives the flexibility to add functionalities to the metalens while preserving its thinness (hundreds of microns) to make it a complex metasurface device (metadevice). Note that only metasurfaces with demonstrated biological experiments are covered in this section. Metasurfaces with a potential for bioapplications are covered in Section 5. Future Directions.

\subsection{Chiral imaging}

Biological analytes, such as amino acids, enzymes, glucose, collagen, etc. possess intrinsic chirality (or handedness) which means they cannot be superimposed on their mirror images like the human hands. Chirality also plays a fundamental role in drug discovery, without the correct chirality, many biomolecules cannot function. Hence, optical chiral imaging based on light polarization is an important research topic in the biology community. Conventionally, it is relying on cascading of multiple optical components in a sophisticated setup which is costly and bulky. Khorasaninejad et al. demonstrated using a single metalens (termed "multispectral chiral lens") to replace the bulky setup and imaged a chiral beetle as shown in Figure 2a, b [96]. The metalens consisted of rectangular-shaped nanofins made of $\mathrm{TiO}_{2}$ with $\mathrm{P}-\mathrm{B}$ phase shifts. It acted on light with different polarizations and separately focused LCP and RCP light at different locations displaying two images of an object with different handedness within the same field of view. Because the beetle's exoskeleton strongly reflects LCP light and absorbs more RCP light, the skeleton structures are more clearly visualized with the LCP image, and the RCP image appears darker. Another metasurface-based polarization imaging technique that includes a compact polarization camera was developed by Rubin et al. [97]. Based on this approach, they were able to acquire images with the full polarization state information, while no traditional polarization optics and moving parts were used. One of the measurement objects was a human face whose 3D features were discernible only from the polarization image taken by the camera but not from the intensity image. This study 
(a)

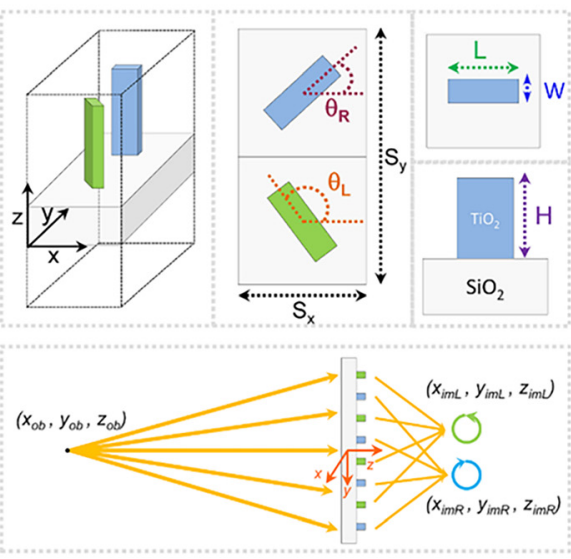

(b)

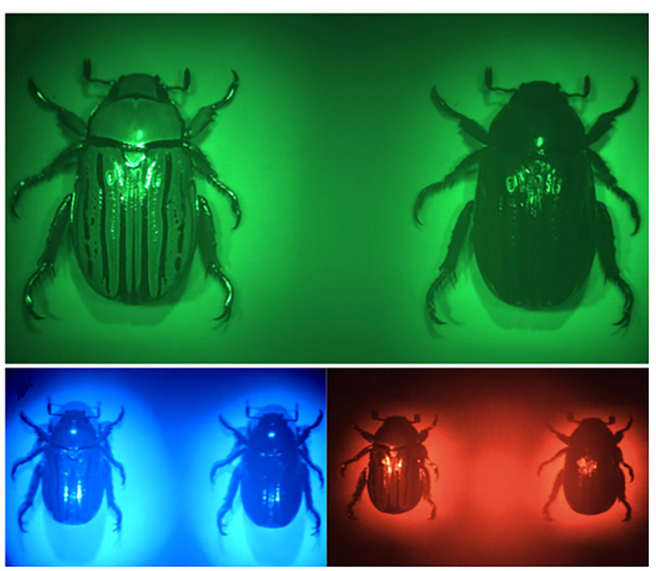

(c)
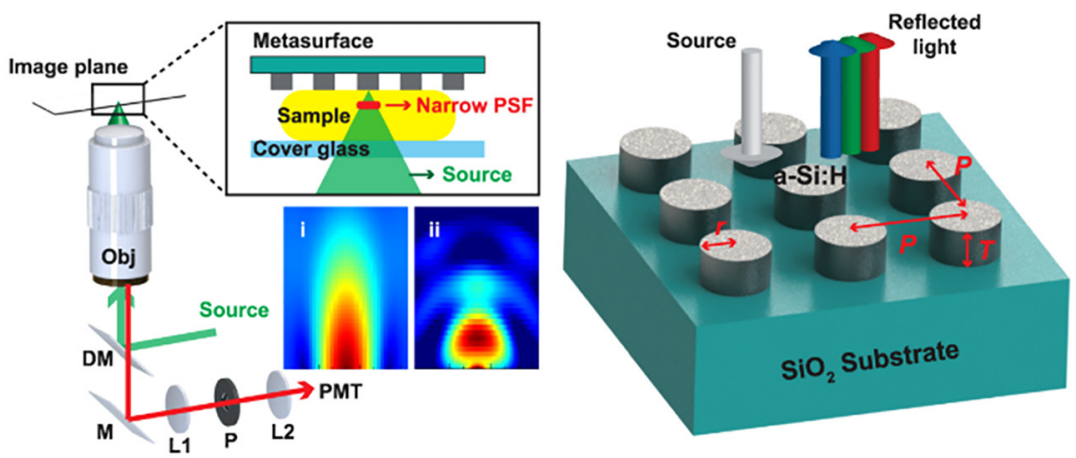

(e)

(d)

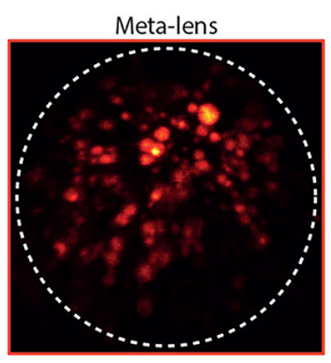

Figure 2: (a) and (b): Chiral imaging; (c)-(e) Fluorescent imaging. (a) (Top row) Perspective view, top view, and side view of the metasurface meta-atoms. (Bottom row) Schematic showing an object is imaged by a metalens which generates two images according to the polarization of the light. The green arrow represents left circularly polarized (LCP) light and blue represents right circularly polarized (RCP) light. (b) A beetle sample imaged by LCP light (left) and RCP light (right) showing different features illuminated with a green, blue, and red LED. (a) and (b) Reproduced with permission from Khorasaninejad et al., Nano Lett. 16, 4595-4600 (2016) [96]. Copyright 2016 American Chemical Society. Direct link: https://pubs.acs.org/doi/abs/10.1021/acs.nanolett.6b01897. Further permissions related to the material excerpted should be directed to the ACS. (c) (Left) Schematic of the metasurface-enhanced axial-narrowing imaging system. Obj: objective lens, DM: dichroic mirror, M: mirror, L1-L2: lenses, P: pinhole. (Right) Schematic of the metasurface consisting of circular-shaped nanodiscs made of a-Si:H. P: period, $r$ : radius, T: thickness. The metasurface reflects a narrow range of wavelengths. (c) Reproduced with permission from Lee et al., Opt. Mater. Express 9, 3248 (2019) [99]. Copyright 2019 Optical Society of America. (d) Schematic showing a two-photon microscope setup with a double-wavelength metalens (DW-ML). Light excitation and collection are from the same position from the sample, i.e. from the same focal plane which is different from a conventional metalens. ML: metalens. (e) Two-photon fluorescent images of microspheres captured by metalens and conventional objective lens. (d) and (e) Reproduced with permission from Arbabi et al., Nano Lett. 18, 4943-4948 (2018) [101]. Copyright 2018 American Chemical Society. 
suggests that the metasurface-based polarization technique could be used in 3D facial recognition and reconstruction.

\subsection{Endoscopic OCT}

High-resolution endoscopic optical imaging is known to be an important tool in biological imaging for studying internal organs. OCT is an imaging technique capable of obtaining tissue microstructures at millimeter depths within the tissue and has been used in ophthalmology, head and neck cancer, cardiovascular diseases, and dermatological clinical diagnosis. OCT uses low-coherence light interferometry configuration to detect the change in the refractive index of the sample and thus offers morphological information. A clinical endoscopic optical imaging device based on OCT integrating a metalens (termed "nano-optics endoscope") was developed [98]. It addressed the spherical aberration and chromatic aberration that are undesirable. The metalens consisted of circularly shaped nanopillars made of amorphous silicon (a-Si) sitting on a $\mathrm{SiO}_{2}$ substrate. It was attached to a prism at a fiber tip encased in an OCT catheter. By studying the optical path of light traveling through the fiber, the prism, the metalens and the catheter sheath reaching the tissue, the authors designed a metalens that achieved both diffraction-limited focusing resolution of $(8.4 \mu \mathrm{m})$ and large depth of focus $(293 \mu \mathrm{m})$ with superior imaging performance than its graded-index lens and ball lens counterparts. Endoscopic OCT imaging in resected human lung specimens and in sheep airways in vivo was demonstrated using the probe.

\subsection{Fluorescent imaging}

Fluorescent imaging of NIH3T3 cells with an enhanced axial resolution was enabled by a metasurface in a laser scanning confocal microscopy configuration [99]. As shown in Figure 2C, the metasurface consisted of an array of a-Si:H nanodiscs working in a reflection mode in the visible wavelength range. The peak reflectance wavelength was tuned by having different radius and period values of the nanodisc array. The enhancement of axial resolution was based on the interference of the excitation and reflection Gaussian beams which was able to confine light beyond the diffraction limit along the axial direction and increase the local electromagnetic field above the metasurface. In order to observe intracellular structures, such as actin filaments, the NIH3T3 cells were stained with red and green fluorescent dyes. Compared to normal confocal images, the metasurface-enhanced confocal images showed a clear distinction between actin filaments (signal) and elliptical shadows of the nuclei (background) and clearer margins of the cells. The axial resolution enhancement was two-fold (around $217 \mathrm{~nm}$ ), and the signal-to-background enhancement was 1.75 fold.

High-resolution and wide field-of-view (FOV) fluorescent imaging of a Giardia lamblia cyst sample was demonstrated with a disorder-engineered metasurface and a spatial light modulator [100]. The goal of the metasurface design as a disordered medium (i.e. diffuser) is to give a farfield pattern that is isotopically scattered over the target angular ranges. One of the advantages of using metasurface for wavefront shaping compared to other types of diffusers is that the disorder of metasurface can be designed, so the input-output characteristics are known as a priori which saves time for initial characterization. It also has excellent stability and a wide angular scattering angle (up to $90^{\circ}$ ). In particular, it was demonstrated that the proposed system (NA $>0.5$, FOV $\sim 8 \mathrm{~mm}$ ) was able to provide a quality image with the same resolution of a $20 \times$ objective but with a FOV of a $4 \times$ objective and the image quality was maintained for over 75 days.

Two-photon (or multiple-photon) excitation microscopy is a fluorescence imaging technique that utilizes a nonlinear process whereby a fluorophore is excited by two or multiple infrared photons and emits a single photon of higher photon energy in the visible spectrum. Because infrared light can penetrate deeper into the tissue and cause less photodamage, it has advantages over the confocal microscopy that uses single-photon excitation. Recently, a two-photon microscope based on metasurfaces was reported [101]. The novelty is that the metalens was able to focus two wavelengths at the same focal plane (termed "double-wavelength metasurface lens"). The two wavelengths correspond to the excitation and emission wavelength, i.e., 820 and $605 \mathrm{~nm}$, respectively. As shown in Figure 2d, conventional lenses including metalenses typically focus different wavelengths at different focal planes due to the chromatic aberration which may compromise imaging quality. The metalens consisted of rectangular-shaped polycrystalline silicon ( $\mathrm{p}-\mathrm{Si}$ ) nanoposts on a fused silica substrate embedded in a SU-8 layer. As a proof of concept, the authors used fluorophore-coated polyethylene microspheres as imaging objects and achieved comparable imaging performance as the conventional objective lens counterpart, as shown in Figure 2e. Almost all fluorescent clusters are visible in both cases, but the metalens is much more compact and easy to integrate with other optical components, such as a dichroic mirror. 
Areas of improvement and exploration were also discussed such as using a metasurface with a double layer to correct for off-axis aberration and the effect of using high power pulsed laser sources. Recently, an endoscopic version of the double-wavelength metalens was proposed [102]. The metalens was designed to focus an excitation wavelength at $915 \mathrm{~nm}$ and collect with an emission wavelength at $510 \mathrm{~nm}$ at the same focal spot with an NA $=0.895$ in a mouse brain environment. Based on theoretical calculations, the lateral resolution can reach a value of $0.42 \mu \mathrm{m}$.

\subsection{Super-resolution imaging}

Super-resolution imaging beyond the diffraction limit can greatly improve image resolution and allows tiny biological features to be seen optically without the need for electron microscopy. Techniques such as near-field scanning optical microscopy [103], stimulated emission depletion microscopy (STED) [104], structured illumination microscopy (SIM) [105], and others have been developed over the years. In particular, SIM utilizes light interference patterns to improve the resolution and is uniquely suitable for wide-field biological imaging with high speed. The recently developed plasmonic structured illumination microscopy (PSIM) explores the interference of the counter-propagating surface plasmon waves of adjacent metallic slits of subwavelength dimensions [106, 107]. The tuning of the interference pattern was achieved by changing the light incident angle. As the angle changes, the interference pattern shifts laterally. This expands the detectable spatial frequency range in the Fourier domain which translates to a resolution improvement of 2.6-fold. An improved version of the PSIM system was later developed by utilizing localized plasmonic structured illumination microscopy (LPSIM) [108, 109]. Instead of using propagating wave interference in PSIM, LPSIM makes use of the tight confinement of localized plasmonic fields at the surface. The structure was a metasurface consisting of a 2D hexagonal array of silver nanodiscs embedded in glass (Figure 3a). Near-field patterns generated by the metasurface under different illumination conditions were studied to optimize the design. Imaging of neuron cells expressing Fzd3-TdTomato was demonstrated [108]. With a high-speed camera, video imaging at $4 \mathrm{~Hz}$ of microtubules over a $28 \times 28 \mu \mathrm{m}$ FOV under a low laser illumination power was demonstrated. The diffraction-limited images and super-resolution images are shown in Figure 3b and c, respectively. The LPSIM system could image subcellular features with a resolution on the order of $\sim \lambda / 11$ [109]. The full width at half maximum (FWHM) of the line profiles show an improvement from 275 to $75 \mathrm{~nm}$ (Figure 3d). With a high spatial-temporal resolution, LPSIM has proved the potential for monitoring the dynamics of proteins in the cell membranes of neurons and other tissues. Due to the evanescent nature of localized surface plasmons, LPSIM is best applied to image very thin objects or at the surface of a thick object. An alternative is to use a hyperbolic metamaterial assisted illumination which uses a metallic/dielectric alternating layered structure to achieve super-resolution imaging at the far-field [110]. A resolution of $80 \mathrm{~nm}$ which is sixfold resolution enhancement of the diffraction limit has been achieved with an $\mathrm{Ag} / \mathrm{SiO}_{2}$ multilayer structure.

\subsection{Magnetic resonance imaging}

Metasurfaces have also been used in other modalities of biomedical imaging. MRI is a medical imaging technique that uses strong magnets and radio waves to generate images of the anatomy and the physiological processes of the body or an animal subject. It has been widely used to study different organs especially the brain for neuroimaging. One of the drawbacks of the MRI scan process is the long scanning time which makes the patient uncomfortable. The time required can be reduced by improving the signalto-noise ratio (SNR) which is an important parameter for characterizing the image quality. Many approaches have been proposed to improve the MRI characteristics including receive coil optimization, the use of special contrast agents, and the use of high dielectric materials. Recently, metasurfaces have been explored to enhance MRI quality. In contrast to other techniques, metasurfaces can controllably redistribute the radiofrequency electromagnetic field at the subwavelength level and hence increase the SNR. By studying the electromagnetic field distribution at different excitation frequencies, one can locally enhance only the magnetic field strength and suppress the electric field. Slobozhanyuk et al. [111] demonstrated that by placing a metasurface pad underneath a fish sample with a 1.5 Tesla (T) MRI system, the SNR was increased by more than two times and the scanning time was reduced by 10 times. The metasurface consisted of an array of metallic wires in a water base holder. A tunable metasurface version was proposed which can tune the resonance frequency of the metasurface pad to match that of the MRI system [112]. This was achieved by changing the water level and thus change the effective permittivity. An SNR improvement of seven times was reported and imaging experiment with grapefruit was conducted with a $1.5 \mathrm{~T}$ MRI system. The homogeneity of the enhancement region was improved as well. Not only plant samples, but an in vivo experiment with the human brain with a $7 \mathrm{~T}$ MRI 
(a)

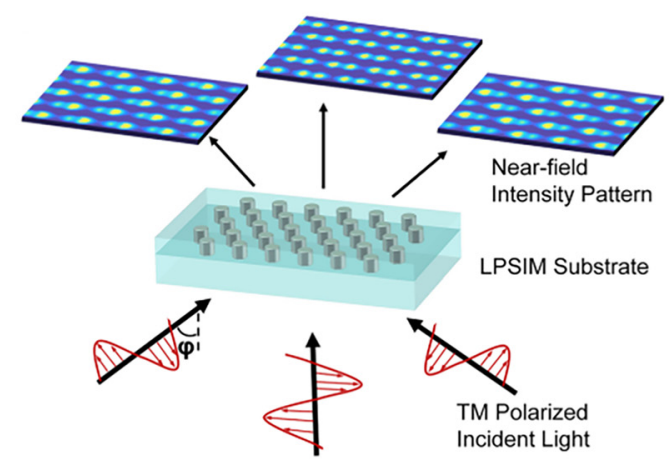

(e)

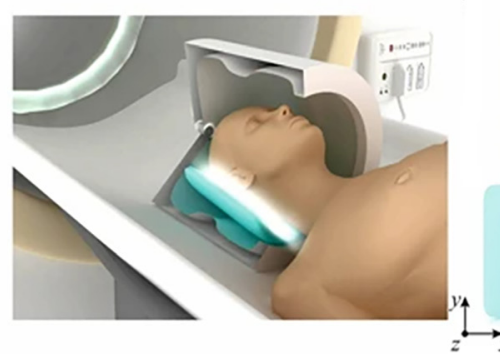

(b)

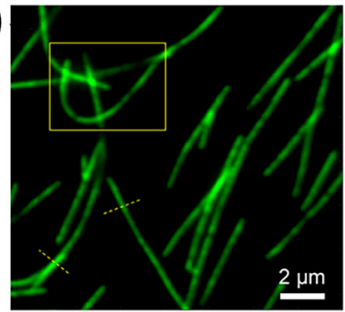

(c)

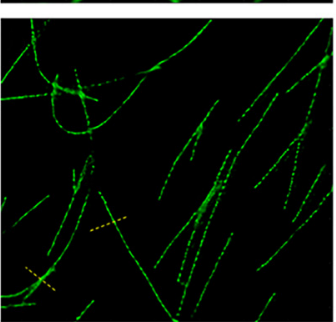

(d)
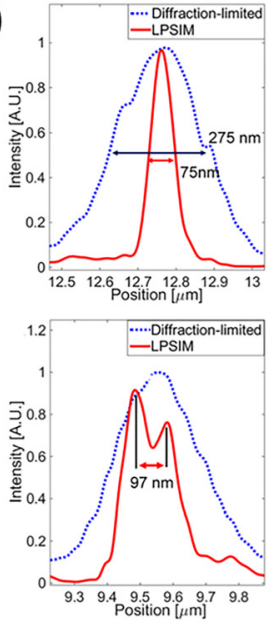
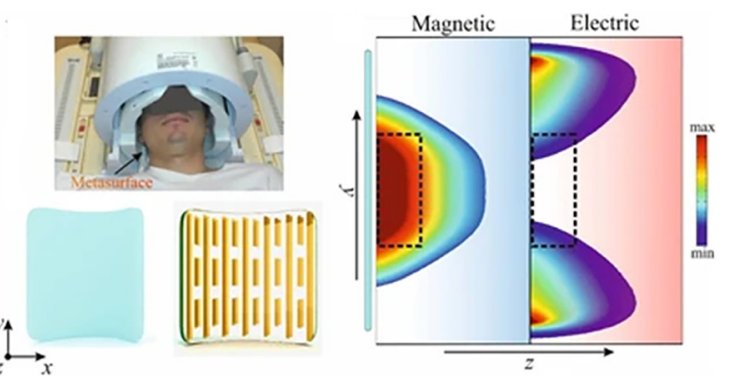

Figure 3: (a)-(d): Super-resolution imaging; (e) magnetic resonance imaging (MRI) imaging. (a) Near-field intensity distributions generated by the metasurface under the illumination of trasnverse-magnetic (TM)-polarized light at an angle of $-60^{\circ}, 0^{\circ}$, and $60^{\circ}$, along the symmetry axes of the localized plasmonic structured illumination microscopy (LPSIM) substrate. (b) One frame of the video recording of green microtubules with diffraction-limited imaging and (c) with super-resolution imaging. (d) Reduced full width at half maximum (FWHM) of the super-resolution imaging compared to diffraction-limited one along the two dashed yellow lines in (b) and (c). (a)-(d) Reproduced with permission from Bezryadina et al., ACS Nano 12, 8248-8254 (2018) [109]. Copyright 2018 American Chemical Society. (e) Illustration and photograph of the experiment showing an MRI scan for a human brain with a metasurface consisting of a high permittivity dielectric pad and metallic stripes. Numerical simulation showing that metasurface can locally redistribute the magnetic field and electric field. (e) Reproduced with permission from Schmidt et al., Sci. Rep. 7, 1678 (2017) [113]. Licensed under Creative Commons Attribution 4.0 International License.

system has also been reported, as shown in Figure 3e [113]. The metasurface pad consisting of carefully designed conductor strips and a high permittivity pad was made flexible and ultrathin such that it was able to fit underneath a person's head and a close-fitting receive coil array. An average enhancement factor was about two times. Other experiments include SNR improvement on in vivo imaging of a human wrist with a 1.5 T MRI system $[114,115]$.

\subsection{Quantitative phase imaging}

QPI is a label-free technique that measures the phase shift quantitatively when light passes through a more optically dense object. It is especially useful to image translucent objects, such as a living human cell which absorbs and scatters little light and difficult to observe in an ordinary optical microscope. Another advantage of QPI is that it is complementary to fluorescence microscopy that uses labels, thus exhibiting lower phototoxicity without photobleaching [116]. The QPI generates phase images instead of intensity images. The phase information obtained provides quantitative information about the morphology and dynamics of individual cells and is sensitive to their thickness and relative refractive index. A typical QPI system is built on a conventional microscope with additional optical components to obtain and analyze the interference patterns, hence it is fundamentally difficult to miniaturize. Recently, a metasurface version of the QPI system with a footprint in the order of $1 \mathrm{~mm}^{3}$ was demonstrated [117]. It was based on two cascaded metasurface layers made of dielectric materials. The first metasurface layer captured two images for transverse electric and transverse magnetic polarizations and redirected them in three different directions to the second metasurface layer, where three birefringent off-axis metalenses were located. After the second metasurface layer, 
three interference contrast images with phase offsets were measured and combined to form the final phase image. In this way, the quantitative phase image was obtained in a single shot. A sea urchin cell was imaged. The phase sensitivity was $92.3 \mathrm{mrad} / \mu \mathrm{m}$ and the lateral resolutions were 2.76 and $3.48 \mu \mathrm{m}$ which made it possible to observe detailed morphology inside the cell. It is impressive that metasurface enables miniaturization of the system to such a small footprint yet can achieve single-cell resolution.

\section{Biosensing}

In the past decade, there has been a crucial need for a rapid and reliable method for molecular binding monitoring, cell study, and cancer detection, while the existing methods, such as PCR, enzyme-linked immunosorbent assay, and mass spectrometry are time-consuming and require delicate experts [118]. Metasurface biosensors consisting of plasmonic and/or dielectric nanostructures provide a novel analytical alternative with the advantages of rapid, label-free, and sensitive detection for various biological samples. There are existing review papers dedicated to the design and applications of plasmonic biosensing such as surface-enhanced Raman scattering biosensors [119], surface plasmon resonance (SPR) biosensors [120, 121], localized surface plasmon resonance biosensors [122], optical fiber integrated biosensors [123-125], and microfluidics integrated biosensors [126]. Hence we have made the scope of this review paper to metasurface biosensing at the molecular and cellular levels with a focus on results that have appeared after the first introduction of the metasurface concept [8]. This section covers metasurface biosensors developed and applied for the targeted detection of antibodies and proteins [127-136], DNAs [138-143], cells [141-145], and cancer biomarkers [146-149]. Table 1 summarizes the performance of different metasurface

\section{Box 2 Performance factors of metasurface biosensors}

\section{Bulk RI sensitivity}

As mentioned earlier, the main principle of the metasurface biosensor is its ability to detect changes in the refractive index (RI). This is determined by the bulk RI sensitivity $\left(S_{B}\right)$, which is given by the following equation [196]:

$$
S_{B}=\frac{d \lambda_{r}}{d n_{B}}
$$

where $\lambda_{r}$ represents the wavelength where the SPR occurs and $n_{B}$ represents the RI of the medium that is in contact with the sensor. The $S_{B}$ is dependent on several factors such as the type of electromagnetic (EM) modes (decay length), resonant wavelength, excitation geometry, and the individual properties of the substrate.

The localization of the EM mode is one of the biggest contributing factor affecting $S_{B}$. Based on the following papers $[197,198]$, delocalized modes yields higher $S_{B}$ values as opposed to localized modes [197, 199, 200]. By using gold films structured with propagating surface plasmon (PSP), it can produce $S_{B}$ values far superior than those which are produced by localized surface plasmon (LSP) when excited on gold nanoparticles [197] and on a gold film [198].

Another factor that affects $S_{B}$ is the resonant wavelength $\left(\lambda_{r}\right)$, as the resonant wavelength increases, so does the $S_{B}$. Research has shown that the shape of the nanoparticle will not affect the $S_{B}$ [201], but the size [161] and sharpness of the edges [162] of the nanoparticles will. For example, the increasing size of the bipyramid nanoparticles increased the sensitivity, and the less sharp of the edges on polyhedral nanoparticles, the poorer the sensitivity.

\section{Figure of Merit (FOM)}

The $S_{B}$ is used to determine the change in RI, it is proportionate to the ability of the sensor to detect minute changes in RI and it is also inversely proportionate to the width $(w)$ of the spectral dips and peaks that are being measured. The features when combined yield the FOM. It allows the quantification of the sensing potential of different nanostructures, it is given by the following equation [196]: 


$$
\mathrm{FOM}_{B}=\frac{S_{B}}{w}
$$

PSP sensors can achieve a higher FOM compared to LSP sensors based on results from these papers [197, 199, 200] because of its narrower spectral features. However, FOM does not entirely translate to better performance as seen in [197], when the FOM for LSP excited on gold nanoparticles reached a maximum value at a wavelength of $700 \mathrm{~nm}$. Its performance outperformed that of PSP sensors by $15 \%$ with the potential to increase performance further by three fold if the access to the sensing surface for the target molecules is improved.

\section{Limit of Detection (LOD)}

The surface coverage resolution, $\sigma_{T}$, determines the sensitivity of the metasurface biosensor to detect the analyte captured on the surface. It can also be seen as the minimum change that occurs when molecules are bound on to the surface, this can be expressed as [196]:

$$
\sigma_{T}=\frac{\sigma_{S O}}{S_{T}}
$$

where $\sigma_{S O}$ is the standard deviation and $S_{T}$ is the sensitivity of the molecular mass bound to the sensor surface. However, this equation does not take into account the analyte transport of the molecules from the solution. Hence, it is necessary to define the LOD. It is the best method to represent the sensitivity of the biosensor. LOD represents the smallest concentration that can be detected by the metasurface biosensor. This is usually determined by how sensitive a sensor is based on three standard deviations of blank measurements, it is expressed as [196]:

$$
\mathrm{LOD}=\frac{3 \sigma_{b}}{S_{c}}
$$

where $S_{c}$ represents the change in the sensor output divided by the change in concentration that results in the change in sensor output. It is dependent on the $S_{T}$, which is the rate that the analyte is being transferred from the solution to the surface of the sensor and the required energy for the interaction between the analyte and the binding substrate.

biosensors mentioned in this section. Box 2 serves as a reference guide for performance factors of evaluating a biosensor.

\subsection{Antibodies and proteins}

Biosensing with metasurfaces is closely related to surface plasmon polaritons (SPPs), which has been widely used for such applications [150-159]. However, the 2D periodic structures of metasurfaces can allow lower radiative damping loss at resonance and enable high quality factor detection with Fano resonance and plasmonic induced transparency [160]. Metasurfaces are also capable to provide multiresonance responses that are difficult to achieve with conventional SPP sensors [160]. Kabashin et al. [127] reported a pioneer work on metasurface biosensing using gold nanorods with a prism coupler. Both guided and longitudinal plasmonic modes were excited at the nanorods sensing layer, which allowed the sensor to provide enhanced sensitivity and narrow figure of merit (FOM) performance. The nanorods also offered a larger surface area for biomolecular sensing, which significantly improved the detection sensitivity. The sensor has been applied for the detection of streptavidin-biotin binding and the detection limit was found to be $300 \mathrm{nM}$. The performance of metasurface-based sensors was further improved by using an asymmetric split-ring array [128]. It excited the low-loss quadrupole and Fano resonance with a narrow linewidth, which showed an order of magnitude enhancement in the quality factor over planar terahertz metamaterials. The sensor sensitivity was found to be $7.75 \times 10^{3}$ and $5.7 \times 10^{4} \mathrm{~nm} / \mathrm{RIU}$ for the quadrupole and Fano resonance, respectively.

A tunable metasurface biosensor consisting of a gold nanoantenna array and a graphene layer has recently been reported by Yu's research group [129, 130]. This operates in the mid-infrared spectral range and provides both quantitative measurement and fingerprint structure information for biomolecules. The structure of the active metasurface is described in Figure 4a. Gold nanorod antennas were fabricated on a graphene layer and a gate voltage was applied to tune the Fermi level of the graphene. A $400 \mathrm{~nm}$ $\mathrm{SiO}_{2}$ layer and a Pt back mirror were deposited below the nanostructure. It supports two hybrid plasmon-phonon modes shown as dips in the reflectance spectra (Figure 4b 
Table 1: Summary of metasurface biosensors.

\begin{tabular}{|c|c|c|c|c|}
\hline Detection sample & Sample type & Nanostructure & Performance & Reference. \\
\hline Streptavidin-biotin binding & Small molecule & Random gold nanorod & $300 \mathrm{nM}$ & [127] \\
\hline Human antibody immunoglobulin G (IgG) & Antibodies & $\begin{array}{l}\text { Gold nano-antenna } \\
\text { array }\end{array}$ & $30 \mathrm{pM}$ & [129] \\
\hline Mouse IgG (M-IgG) & Protein & Elliptical zigzag array & 3 molecules $/ \mu \mathrm{m}^{2}$ & [131] \\
\hline Protein $\mathrm{A} / \mathrm{G}$ monolayer & Protein & Elliptical zigzag array & 2130 molecules $/ \mu \mathrm{m}^{2}$ & [132] \\
\hline $\begin{array}{l}\text { Insulin, vascular endothelial growth factor (VEGF), } \\
\text { and thrombin biomarkers }\end{array}$ & Protein & $\begin{array}{l}\text { Al nanodisks-in- } \\
\text { cavities array }\end{array}$ & $1 \mathrm{fmol}$ in a $10 \mu \mathrm{L}$ droplet & [133] \\
\hline HIV envelope glycoprotein & Protein & Nanograting & Nil & [134] \\
\hline Immunoglobulin G (IgG) & Protein & Nanowire array & $300 \mathrm{pM}$ & [135] \\
\hline Streptavidin-biotin binding & Small molecule & $\begin{array}{l}\text { Metallic nanogroove } \\
\text { array }\end{array}$ & $1 \mathrm{fM}$ & [136] \\
\hline $\begin{array}{l}\text { DNA aptamer-human odontogenic ameloblast- } \\
\text { associated protein (ODAM) binding }\end{array}$ & Protein & Elliptical zigzag array & $0.2 \mathrm{pg} / \mathrm{mm}^{2}$ & [138] \\
\hline $\begin{array}{l}\text { DNA aptamer-fructose, chlorpyrifos methyl and } \\
\text { thrombin binding }\end{array}$ & Molecule & Gold patch array & $0.2 \mathrm{ng}$ & [139] \\
\hline DNA-DNA molecular binding & DNA & $\begin{array}{l}\text { Three-arm } \\
\text { nanoantennas }\end{array}$ & Nil & [166] \\
\hline ssDNA & DNA & $\begin{array}{l}\text { Graphene-gold } \\
\text { metasurface }\end{array}$ & $1 \mathrm{aM}$ & [167] \\
\hline Epithelium derived oral cancer cell (HSC3) & Oral cancer cell & $\begin{array}{l}\text { Concentric gold } \\
\text { nanorings }\end{array}$ & Nil & [144] \\
\hline GABA neurotransmitter & Cell & Gold nanodipoles & Nil & [145] \\
\hline Lipid membrane-streptavidin & $\begin{array}{l}\text { Cell lipid } \\
\text { membrane }\end{array}$ & $\begin{array}{l}\text { Gold nanoantenna } \\
\text { array }\end{array}$ & Nil & [141] \\
\hline $\begin{array}{l}\text { Fungi, Penicillium chrysogenum, (penicillum), and } \\
\text { Bacteria, Escherchi coli (E. coli) }\end{array}$ & Bacteria & Gold square rings & $10^{7}$ units $/ \mathrm{ml}(E$. coli $)$ & [143] \\
\hline Epidermal growth factor receptor 2 (ErbB2) & $\begin{array}{l}\text { Breast cancer } \\
\text { biomarker }\end{array}$ & Silicon nanopost array & $0.7 \mathrm{ng} / \mathrm{ml}$ & [146] \\
\hline $\begin{array}{l}\text { Alpha-fetoprotein (AFP) and Glutamine transferase } \\
\text { isozymes II (GGT-II) }\end{array}$ & $\begin{array}{l}\text { Liver cancer } \\
\text { biomarker }\end{array}$ & Gold split ring & $\begin{array}{l}0.02524 \mu \mathrm{g} / \mathrm{ml} \text { (AFP) and } \\
5 \mathrm{mu} / \mathrm{ml}(\mathrm{GGT}-\mathrm{Il})\end{array}$ & [147] \\
\hline Epithelium-derived oral cancer cell (HSC3) & Oral cancer cell & $\begin{array}{l}\text { Gold double split ring } \\
\text { array }\end{array}$ & $900 \mathrm{kHz} /$ cell ml $^{-1}$ & [148] \\
\hline
\end{tabular}

GABA, gamma-aminobutyric acid; AFP, alpha-fetoprotein.

and c). The biosensor can also operate in a passive mode without the graphene layer (Figure 4d). Human antibody immunoglobulin $\mathrm{G}$ ( $\operatorname{IgG}$ ) was used to evaluate the performance of the metasurface biosensor. Protein A/G was attached to the gold nanoantennas as the capture agent because it contains both protein A and protein $\mathrm{G}$ binding domains which have a high affinity with the fragment crystallizable region of the $\mathrm{IgG}$, this would reduce nonspecific biding allowing better sensitivity and specificity. The results show that as the IgG concentration increased, the resonance shifted to a lower wavelength. Two other peaks were also observed when the intensity increased with the increase in IgG concentration. These two peaks should be assigned as amide I and amide II (Figure 4e). The shift in resonance and the change in intensity of the amide peaks show a strong correlation that the change was due to IgG binding rather than nonspecific binding. The detection limit was $30 \mathrm{pM}$. Physiologically, it means the device can detect biomarkers for colon cancer which is $10 \mathrm{nM}$ [161]. However, the value still needs to be improved as the detection limits of biomarkers for prostate cancer and ovarian cancer are 1 [162] and 4 pM [163], respectively. The metasurface has further been applied for surface-enhanced Fourier transform infrared (FTIR) measurement for IgG molecules. The measurement results show that it can provide four orders of magnitude improvement in the detection sensitivity compared to conventional attenuated total reflection FTIR method (Figure 4f).

Metasurfaces has also been combined with hyperspectral imaging for ultrasensitive biosensing by Altug's research group [131]. In this case, the metasurface consisted of an array of zigzag structures arranged in pairs. The pairs of nanobars were tilted along the $y$-axis in an asymmetrical manner, which increased the resonance for strong light trapping making it optimal for biosensing and 
(a)

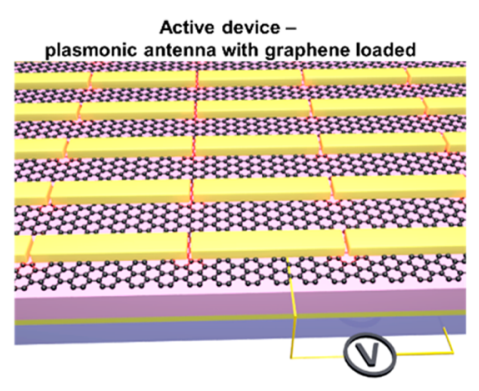

(d)

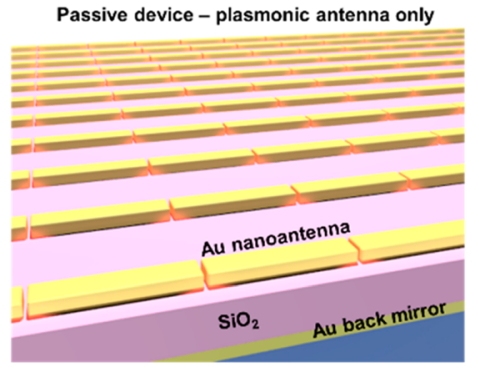

(b)

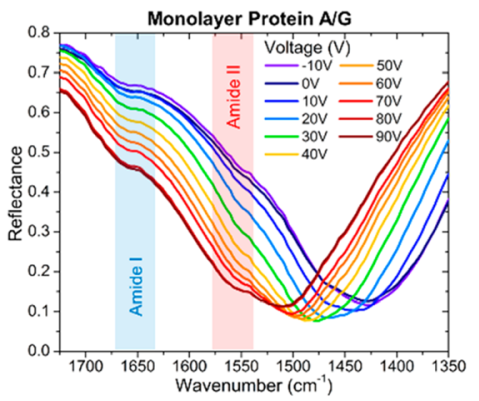

(e)

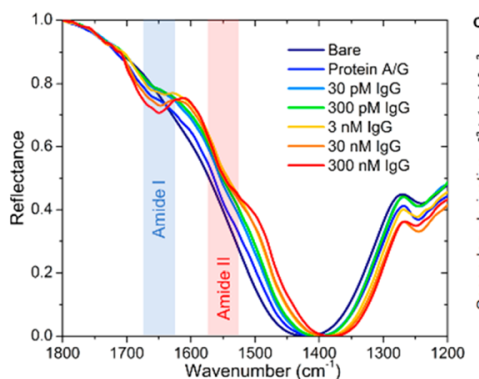

(c)

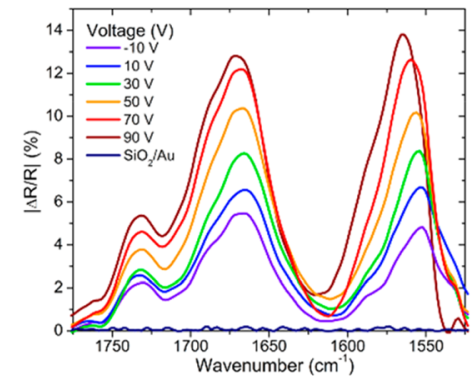

(f)

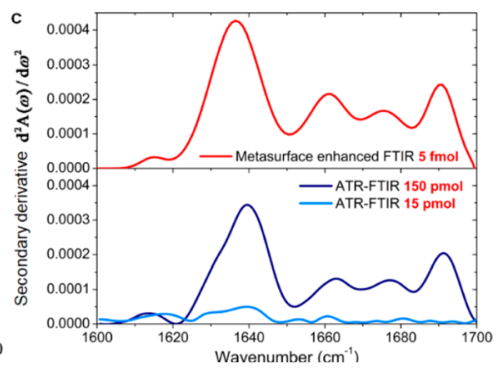

Figure 4: A tunable metasurface biosensor consisting of a gold nanoantenna array and a graphene layer for IgG antibodies detection. (a) Schematic of the active metasurface sensor with a graphene tunable layer. The Fermi level is tuned by an applied gate voltage. (b) Reflectance spectra for the measurement of the protein $\mathrm{A} / \mathrm{G}$ monolayer at different gate voltages. (c) Relative absorption spectra of the amide I and II bands of the protein A/G monolayer at various voltages. (d) Schematic of the passive metasurface sensor. Gold nanorod antennas were fabricated on the $400 \mathrm{~nm} \mathrm{SiO}$ thin film and a gold back mirror, while the gap between each nanorod antennas is $30 \mathrm{~nm}$. (e) Measured reflectance spectra for the protein $A / G$ monolayer and the molecular binding with different concentrations of IgG antibodies. (f) Surfaceenhanced Fourier transform infrared (FTIR) measurement for IgG molecules. The measurement results show that it can provide four orders of magnitude improvement in the detection sensitivity compared to conventional attenuated total reflection FTIR method. (a)-( $f$ ) Reproduced with permission from Li et al., ACS Photonics 6, 501-509 (2019) [129]. Copyright 2019 American Chemical Society.

allowed spectral tunability. In the optical set-up, a continuously tunable bandpass filter was coupled to a supercontinuum laser source to excite the metasurface at different wavelengths and the spectral images were captured by a CMOS camera. The imaging sensor was demonstrated for the molecular binding detection of rabbit antimouse IgG (R-IgG) and mouse $\operatorname{IgG}(\mathrm{M}-\mathrm{IgG})$. By comparing the shift maps of 66 different concentrations of samples, the results showed that three molecules per $\mu \mathrm{m}^{2}$ binding would result in $\sim 0.4 \mathrm{~nm}$ shift in resonance. This is due to the extremely localized fields generated by the tilting of the nanobars. The zigzag array structure was also used in a 2D pixel format to achieve imaging-based spectrometerless molecular fingerprint detection [132]. The resonance position of each pixel was tuned by changing the sizes of the meta-atoms. The 2D spectral barcode system covered the mid-IR range between 1300 and $1800 \mathrm{~cm}^{-1}$, which were located in the spectral absorption regions of protein amide I and amide II functional groups. The system was then demonstrated for protein $A / G$ monolayer detection, where the spectral peaks of amide I $\left(1650 \mathrm{~cm}^{-1}\right)$ and amide II $\left(1540 \mathrm{~cm}^{-1}\right)$ were measured in the transmission imaging measurement and correlated with the results of the FTIR spectroscopy. The detection capability of the system has further been tested with PMMA, PE, and glyphosate pesticide in order to show its potential for various application fields in biosensing, material science, and environmental monitoring.

Aluminum normally provides weak plasmonic response due to the relatively low electron density compared to noble metals. However, Siddique et al. [133] recently presented a metasurface structure comprising aluminum-based nanodisks-in-cavities array which could achieve 1000-fold fluorescence enhancement in the visible wavelength range. This designed structure generated hybrid multipolar lossless plasmonic modes, which enabled strong electromagnetic field confinement. Through these signal enhancement effects, the metasurface sensor was used for the detection of insulin, vascular endothelial, and thrombin biomarkers. These 
biomolecules with a low concentration of $1 \mathrm{fmol}$ could be detected in a $10 \mu \mathrm{l}$ droplet.

Recently, a DVD-based metasurface has been reported [134], which provides the advantages of cost-effective, large detection area, and multimodal sensing. A multilayer structure of titanium (Ti), silver $(\mathrm{Ag})$, and gold $(\mathrm{Au})$ layers were deposited on a clean DVD surface. The period of the grating structure was $750 \mathrm{~nm}$ and the height was $60 \mathrm{~nm}$. Refractive index sensing of different concentrations of glycerol (1.336-1.428 RIU) has been conducted and the sensor sensitivity was found to be $377.69 \mathrm{~nm} / \mathrm{RIU}$, while the asymmetric Fano resonance peak red-shifted from 524.3 to $553.2 \mathrm{~nm}$. The metasurface was functionalized with an antigp120 HIV antibody and applied for the detection of HIV envelope glycoprotein (gp120, $200 \mu \mathrm{g} / \mathrm{ml}$ ) located at the HIV-1 virus surface. After that, the sensor was used for detecting HIV-1 virus, cumulative resonance wavelength shift was demonstrated at the Fano resonance peak for HIV-1 virus in the concentration of 40 and 80 copies/ $\mu \mathrm{l}$. In addition, the measurement was correlated with SEM images of the HIV-1 virus on the nanograting structures.

A leaky cavity mode resonance (LCMR)-based detection has been demonstrated with a metasurface biosensor consisting of a silicon nanowire array and a graphene monolayer (Figure 5a) [135]. Silicon was used because of its high refractive index, low loss, and its ability to be integrated with current sensing technology. In addition, the silicon nanowire structure was also able to alter the LCMR wavelength; this can be achieved by altering the width and height. This shift in resonance can be readily observed with an optical microscope, i.e. the change in resonance resulted in a change in the color of the metasurface. The color changed progressively from yellow to orange then red as the spacing between the silicon arrays increased. The metasurface was tested with the IgG protein, protein $A / G$ was used as a binding agent. The functionalized graphene allowed for better binding of IgG on the metasurface. Different concentrations of IgG were prepared in the phosphate-buffered saline (PBS) solution and tested using the biosensor (Figure $5 b$ ). The results indicated that the increase in $\operatorname{IgG}$ resulted in a redshift of the resonance. The difference in performance was also prominent when comparing the graphene functionalized surface and the silicon surface. The graphene functionalized surface has a lower detection limit of $300 \mathrm{pM}$, while the silicon surface showed a resonant shift below the noise level when the concentration of the IgG was around 30 and $100 \mathrm{nM}$. This shows that silicon binds poorly with biomolecules as the number of the binding site that was present was low after washing with PBS after the incubation phase. (a)

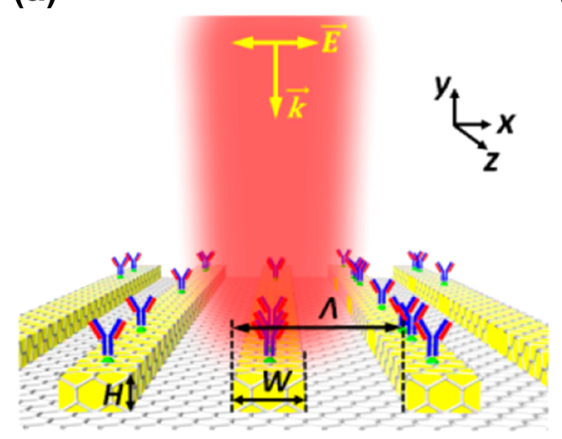

(c)

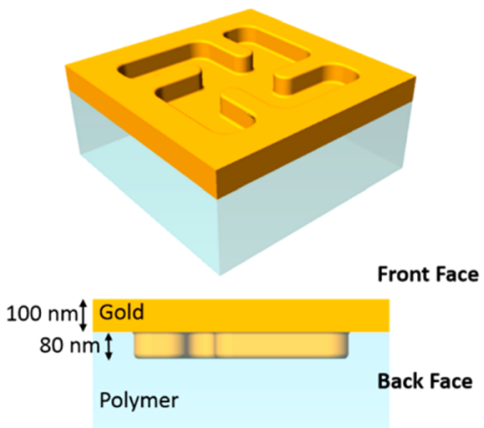

(b)

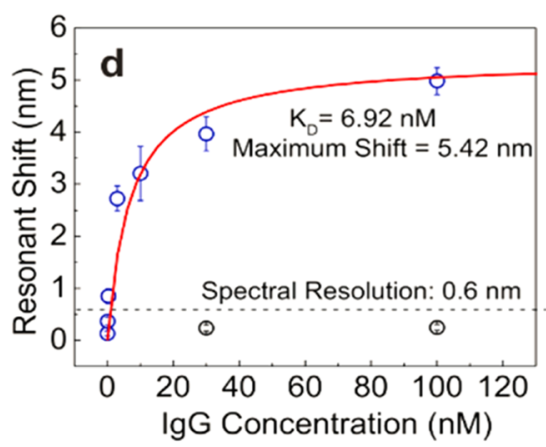

(d)
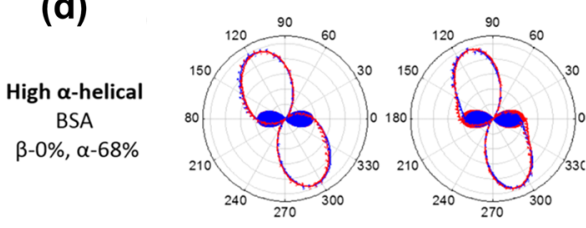

High $\beta$-sheet Concanavalin A $\beta-46 \%, \alpha-2 \%$

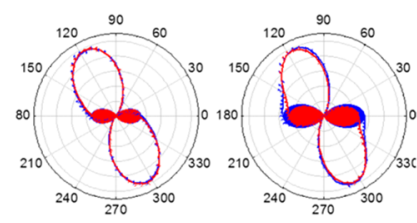

Figure 5: Antibodies and protein detection. (a) Schematic of the leaky cavity mode resonance biosensor with periodic silicon nanowires (SiNWs). A graphene monolayer is overlaid on the SiNW array for the adsorption of biomolecules. (b) The resonant wavelength shift for the molecular bindings between $A / G$ protein and different concentrations of IgG antibody. The detection limit was found to be $300 \mathrm{pM}$. (a) and (b) Reproduced with permission from Guo et al., ACS Photonics 1, 221-227 (2014) [135]. Copyright 2014 American Chemical Society. (c) Schematic of the chiral solid-inverse metasurface structure. (d) Polar plots for bovine serum albumin (BSA) and concanavalin A proteins detection with the chiral metasurface. (c) and (d) Reproduced with permission from Jack et al., Nano Lett. 16, 5806-5814 (2016) [137]. Copyright 2016 American Chemical Society. Direct link: https://pubs.acs.org/ doi/10.1021/acs.nanolett.6b02549. Further permissions related to the material excerpted should be directed to the ACS. 
In addition, a hyperbolic nanogroove metasurface was developed for bovine serum albumin (BSA)-biotin binding detection [136]. The metasurface used a metallic nanogroove structure on metal. Six patches of nanogroove arrays were carved into the metal with each patch containing $5 \times 5$ nanogrooves. The metasurface was tunable by altering the width and the period of the groove; in this experiment, a period of $150 \mathrm{~nm}$ and a width of $30 \mathrm{~nm}$ were used. Calibration of the sensing platform was performed using glycerol of different concentrations. The change of the differential phase was up to $36.448^{\circ}$, this corresponded with the refractive index of 1.3326-1.3338 RIU. This means that the differential phase sensitivity goes up to $30,373 \mathrm{deg} /$ RIU that is five times higher than conventional gold thinfilm sensors. The metasurface was then used to monitor the interaction of BSA in mild to extreme dilutions. The introduction of the BSA led to a change in the phase shift which increased exponentially signifying the increase in binding of the BSA. A washing step with distilled water was performed to remove the BSA resulting in a slight decrease in the phase shift. The higher concentrations of BSA would yield a larger differential change in the phase as there was more BSA present. The detection limit of BSA was found to be $1 \times 10^{-19} \mathrm{M}$. To further investigate the detection ability of the sensing platform, biotin was used. A standard streptavidin-biotin model was adopted, and the streptavidin was functionalized on the metasurface by physisorption forces $[164,165]$. The detection limit for biotin was found to be $1 \times 10^{-15} \mathrm{M}$.

Furthermore, the chiroptical properties of chiral biomaterials have been used for the detection of helical biopolymers and proteins [137]. As shown in Figure 5c, a chiral plasmonic nanostructure with an inverse hybrid gold metafilm was fabricated to sense the reflectivity and chiroptical properties changes of biomacromolecular secondary structures, and it provided specific fingerprints of the $\alpha$-helical, $\beta$-sheet, and disordered motifs. They further demonstrated the measurement of BSA and concanavalin A proteins with polarimetry Figure $5 \mathrm{~d}$. It was a new detection approach that did not rely on refractive index sensing and the detection limit could reach the picogram range.

\subsection{DNA detections}

An angle-multiplexed dielectric metasurface has been introduced for human odontogenic ameloblast-associated protein (ODAM) detection by using single-stranded DNA aptamer for periodontal diseases diagnosis [138]. A zigzag array of elliptical germanium based metasurface was designed for molecular binding detection at the midinfrared region (Figure 6a). In each unit cell, the plasmonic resonance spectral position can be tuned by different incidence angles. During ODAM detection, polylysine was first bound to the metasurface to allow the immobilization of DNA probes and polylysine interacted with DNA via electrostatic interactions. Then, the ODAM molecules were detected by DNA aptamer. The corresponding signal increased at the amide I and amide II bands as shown in Figure 6b. Experimental results showed that the detection limit of the metasurface was 3000 molecules $/ \mu \mathrm{m}^{2}$ that would yield a surface mass density of $0.2 \mathrm{pg} / \mathrm{mm}^{2}$. This value was comparable with the detection limit of the gold standard BIAcore based measurement in molecules detection.

Furthermore, graphene-based metasurface with gold patch resonator array (Figure 6C) were designed for the detection of fructose, chlorpyrifos-methyl, and thrombin with a probe DNA [139]. The graphene layer enhanced the binding of analytes to the metasurface and elicited a stronger response to external molecules with the $\pi-\pi$ stacking when molecules interact with delocalized $\pi$-electrons of graphene (Figure $6 \mathrm{~d}$ ). Chlorpyrifos-methyl has a benzene ring that interacts with the $\pi$-electrons on the graphene, while fructose lacks $\pi$-electrons. Measurement results showed that the reflectance shift of chlorpyrifosmethyl is much larger than that of fructose and the detection limit of chlorpyrifos was $0.2 \mathrm{ng}$ (Figure 6e, f). DNA aptamers were further utilized for thrombin detection. The molecular bindings between DNA aptamers and thrombin produced a $4 \%$ reflectance intensity decrease.

Surface-enhanced fluorescence spectroscopy has been demonstrated with metasurface for DNA-DNA molecular binding detection [166]. The metasurface was designed with gold log-periodic nanoantennas on a silicon dioxide substrate with a three-arm design which allowed the manipulation of circularly polarized waves (Figure $6 \mathrm{~g}$ ) [139]. A thin film of titanium ( $3 \mathrm{~nm}$ ) was used to ensure the adhesion of the meta-atoms to the silicon dioxide substrate. DNA probes labeled with fluorescence tags were then functionalized onto the metasurface with a selfassembled monolayer (Figure 6h). The surface-enhanced fluorescence was excited with a CW diode at $640 \mathrm{~nm}$. Figure $6 \mathrm{i}$ shows the image of the fluorescence intensity of the fluorescence molecules on the meta-atoms. Comparing to the fluorescence intensity of the fluorescence molecules on a gold film, it was shown that meta-atoms enhanced the fluorescent signal.

The graphene-gold metasurface architecture was demonstrated by Zeng et al. [167] to achieve ultrasensitive biosensing. At the metasurface interface, a single (or multilayer) layer of graphene was deposited on a gold 


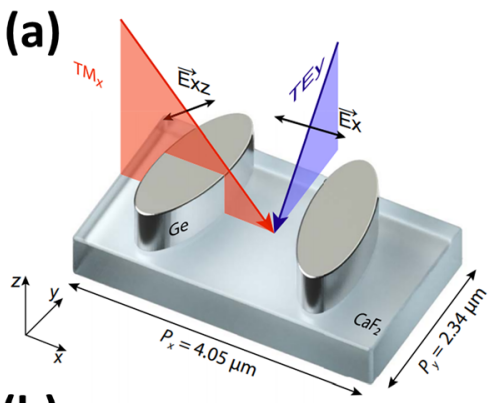

(b)

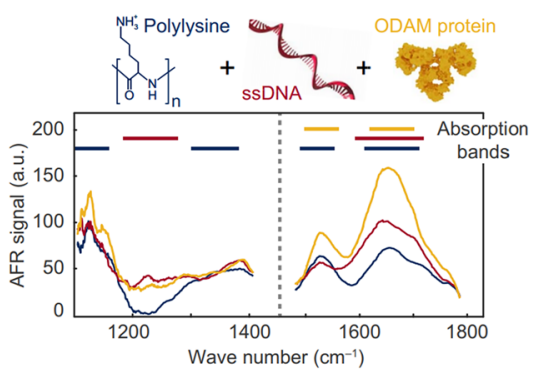

(g)

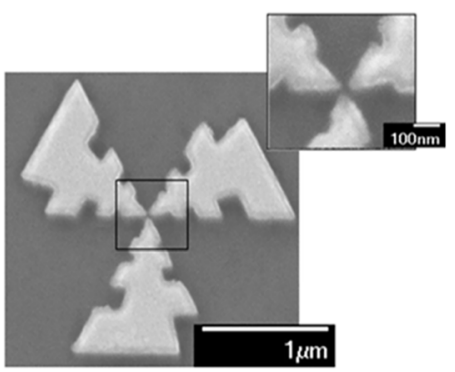

(c)

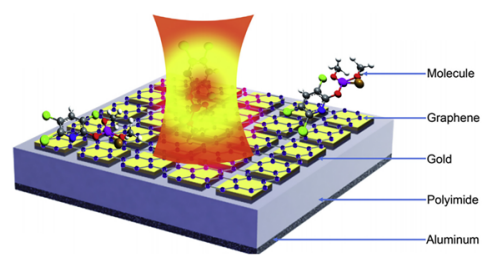

(d)

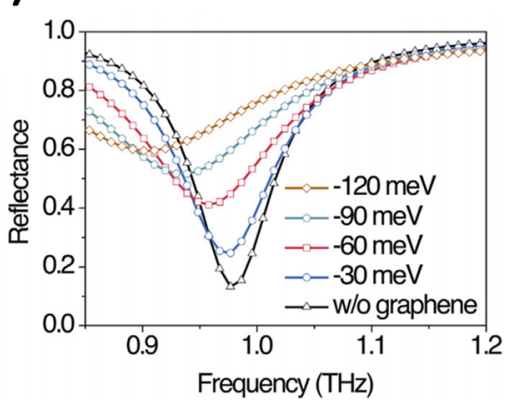

(h)

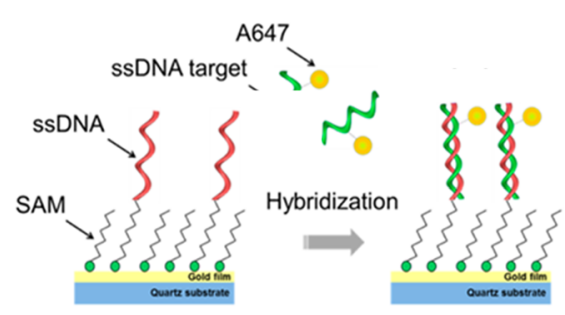

(e)

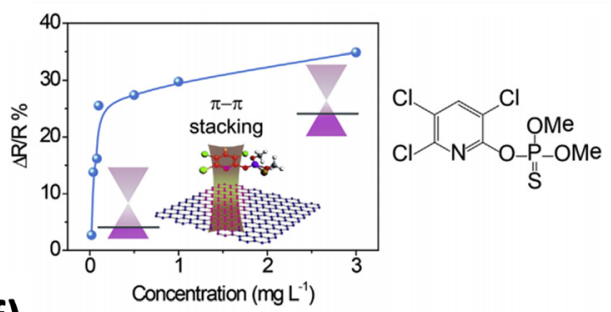

(f)

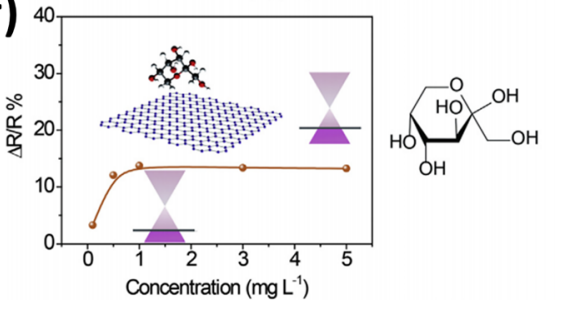

Figure 6: DNA molecules detection.

(a) Design of elliptical zigzag array, while the trasnverse-magnetic (TM) and TE modes vary at different light incident angles. Simulation results show strong near field enhancement surrounding the resonators, which is suitable for signal amplification and molecular vibration detection for analytes. (b) At human odontogenic ameloblast-associated protein (ODAM) detection, the ODAM molecules were detected by DNA aptamer. The corresponding signal increase at the amide I and amide II bands. Experimental results showed that the detection limit of the metasurface was 3000 molecules $/ \mu \mathrm{m}^{2}$ that would yield a surface mass density of $0.2 \mathrm{pg} / \mathrm{mm}^{2}$. (a) and (b) Reproduced with permission from Leitis et al., Sci. Adv. 5, eaaw2871 (2019) [138]. Distributed under a Creative Commons Attribution NonCommercial License 4.0 (CC BY-NC). (c) Gold patch resonator array in 85-95 $\mu \mathrm{m}$ width was fabricated on the polyimide layer ( $4 \mu \mathrm{m}$ thickness) and aluminum back-ground mirror (200 nm). The metasurface was covered by a CVD-grown single graphene layer to form graphene-metamaterial heterostructure for biosensing applications. (d) Numerical modeling of different Fermi levels of the graphene layer ranged from 30 to 120 meV. (e) Reflectance signal for different concentrations of chlorpyrifos-methyl molecules $(0.02-3.0 \mathrm{mg} / \mathrm{l})$. (f) Reflectance signal for fructose molecules (0.1-5.0 mg/ml). Measurement results showed that the reflectance shift of chlorpyrifos-methyl is much larger than that of fructose and the detection limit of chlorpyrifos was $0.2 \mathrm{ng}$. (c)-(f) Reproduced with permission from Xu et al., Carbon N. Y. 141, 247-252 (2019) [139]. Copyright 2019 Elsevier. (g) SEM image of the three-arm log-periodic gold nanoantenna structure. The three-arm design allows polarization independence and it manipulates on circularly polarized waves. (h) DNA probes were attached to the sensor surface to capture Alexa Fluor 647 labeled ssDNA targets. (i) The fluorescence intensity across the nanoantenna array indicates the binding between the probe and DNA target molecules. 4.8 times signal enhancement was recorded with the three-arm nanoantenna structure comparing to a flat gold film. (g)-(i) Reproduced with permission from Aouani et al., J. Phys. Chem. C 117, 18620-18626 (2013) [166]. Copyright 2013 American Chemical Society. 
sensing layer, which allowed a strong localization of the electromagnetic field at the interface. In another approach, gold nanoparticles were used to further enhance the sensor response. The detection limit of ssDNA was $1 \times 10^{-18} \mathrm{M}$. The authors also reported simulation results on a graphene$\mathrm{MoS}_{2}$ hybrid structure [168], which can provide a phase sensor response enhancement factor of greater than 500 .

\subsection{Cells}

Cell apoptosis study is another recent research area with metasurface biosensors. A metasurface biosensor in terahertz was used for in situ cell apoptosis measurement [144]. The design contained a planar array of five concentric gold nanorings $(20,28,36,44$, and $52 \mu \mathrm{m})$ on a polyimide substrate (Figure 7a). The distance between each group of concentric gold nanorings was $4 \mu \mathrm{m}$ and the transmitted terahertz spectrum in Figure $7 \mathrm{~b}$ showed four resonance peaks. In the biosensing experiment, single layer epithelium-derived oral cancer cells (HSC3) and normal epithelial cells were detected using. Cells of different concentrations were prepared and seeded on the sensor surface. As shown in Figure 7C, the cells caused a change in frequency which increased with the increasing concentration. In the cell apoptosis detection experiment, an oral cancer cell (SCC4) was treated with a targeted cancer drug, cisplatin, which induced cell death to the cancer cell. Measurement results showed that the signal of the drug-treated SCC4 cells was slightly decreased against time, while the signals of the control cells were increased by $\sim 50 \%$. It was predicted that the cell multiplication process in SCC4 cell have been suppressed by the cancer drug, which caused a decrease in sensor signal (Figure 7d). While the cells that were not treated with drugs showed an increase in frequency, this can be attributed to the increasing cell concentration as the cells multiply (Figure 7e).

Recently, a multiresonant metasurface that was able to detect lipid membrane and protein interaction via the methylene and amide band was reported [145]. The metasurface contained an array of gold nanoantennas with two nanodipoles as the meta-atom. The resonance frequency can be altered by adjusting the length of the individual dipoles. Altering the periodicity can also result in a change in the metasurface, in this case, the reflectance is altered. The dipole arrays consisted of two arrays, the second array was made slightly different than the first array by having different lengths and periodicity as shown in Figure $7 f$. Several tests were performed to demonstrate the ability of the metasurface for multiple analytes detection. The first test was done with streptavidin and a phospholipid membrane. A $10 \mathrm{~nm}$ silicon dioxide layer was added to create a hydrophilic surface to allow the membrane to form [140]. The biotinylated lipids were used to facilitate the binding of the streptavidin to the membrane. The authors further investigated the interaction between the lipid membrane and melittin, which is a toxic peptide. Melittin has a strong affinity with the lipid membrane which caused the displacement of lipid membranes resulting in the formation of pores. As shown in Figure $7 \mathrm{~g}$, the lipid signal decreased as the melittin signal increased. The metasurface was then to detect small analytes, lipids, and peptides. Cholesterol enriched lipid vesicles with neurotransmitters were used. This was used to simulate synaptic vesicles found in neurons. The vesicles are loaded with gammaaminobutyric acid (GABA), a neurotransmitter for the inhibition of synaptic transmission. As shown in Figure 7h, the injection of the GABA vesicles resulted in an increase in the sensor signal.

Metasurface with a gold nanoantenna array on top of a calcium fluoride substrate [141] was proposed for lipid and protein fingerprints detection (Figure 7i). To demonstrate the detection specificity of the metasurface, a lipid membrane-protein assay has been tested. Lipid vesicles containing 1,2-dioleoyl-sn-glycerol-3-phosphocholine and biotinylated 1,2-Bis(diphenylphosphino)ethane (DPPE) was injected to the metasurface to form a layer of the lipid membrane. Then, streptavidin was injected to the metasurface, and the SEIRA signal was used to detect the molecular binding between streptavidin and the biotin group on DPPE lipids (Figure 7j).

Muhammad et al. [142] demonstrated a voltagetunable metasurface for the detection of cells. The design was with parallel gold nanorods arranged in a cross manner. By altering the length, spacing, density of nanorods, and the applied voltage on the lithium niobate film, the metasurface could be tuned to accommodate different resonant modes. To test the performance of the sensor, solvents (water, acetone, 2-propanol, and chloroform) with different refractive indices were measured. The sensor was further applied for cells and biomaterials detection with different applied voltages and resonance wavelengths.

A metasurface biosensor has further been demonstrated for microorganisms detection [143]. This comprised an array of gold square rings with a nanogap. The plasmonic resonance wavelength of the sensor was affected by the capacitance of the nanogap and also the inductance of the outer ring structure. The metasurface was applied for the detection of Penicillium chrysogenum fungus and Escherichia coli (E. coli) bacteria. The injection of penicillium resulted in a $9 \mathrm{GHz}$ shift in frequency and the sensor 
(a)

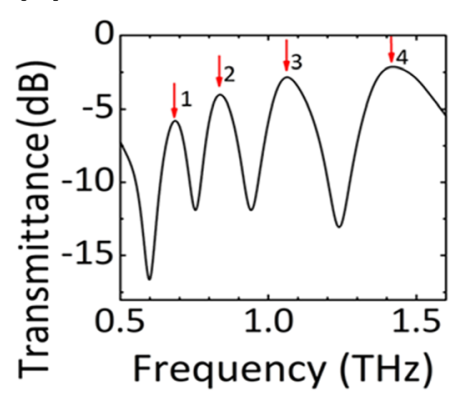

(d)

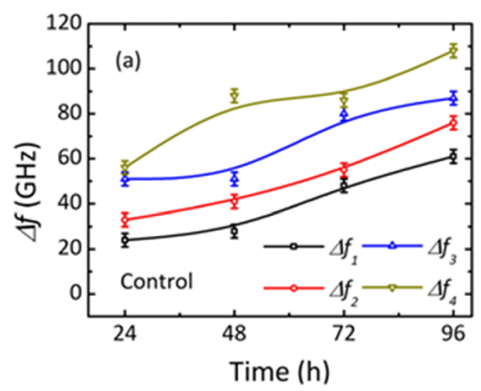

(b)

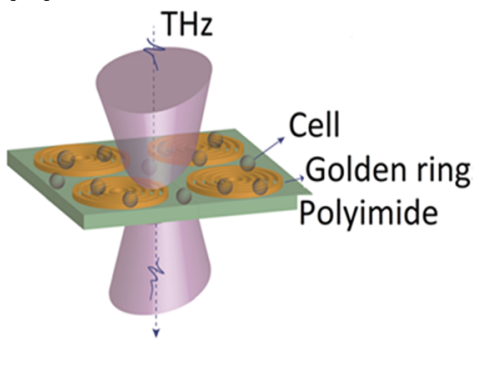

(e)

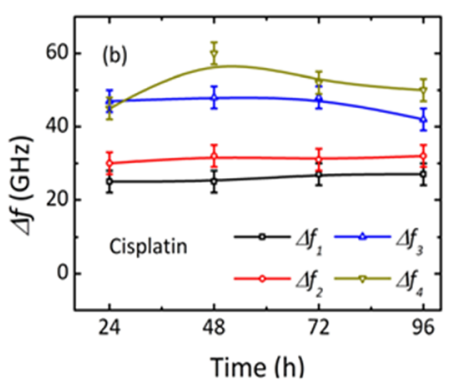

(c)

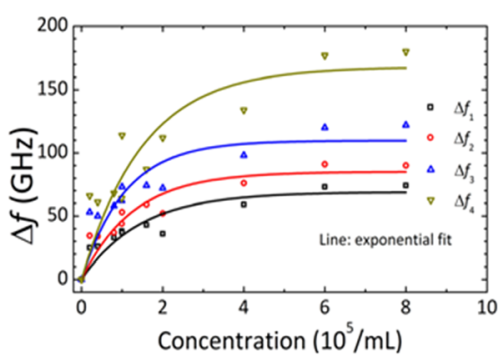

(f)
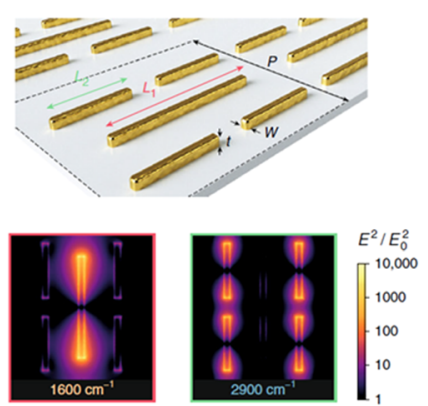

(g)

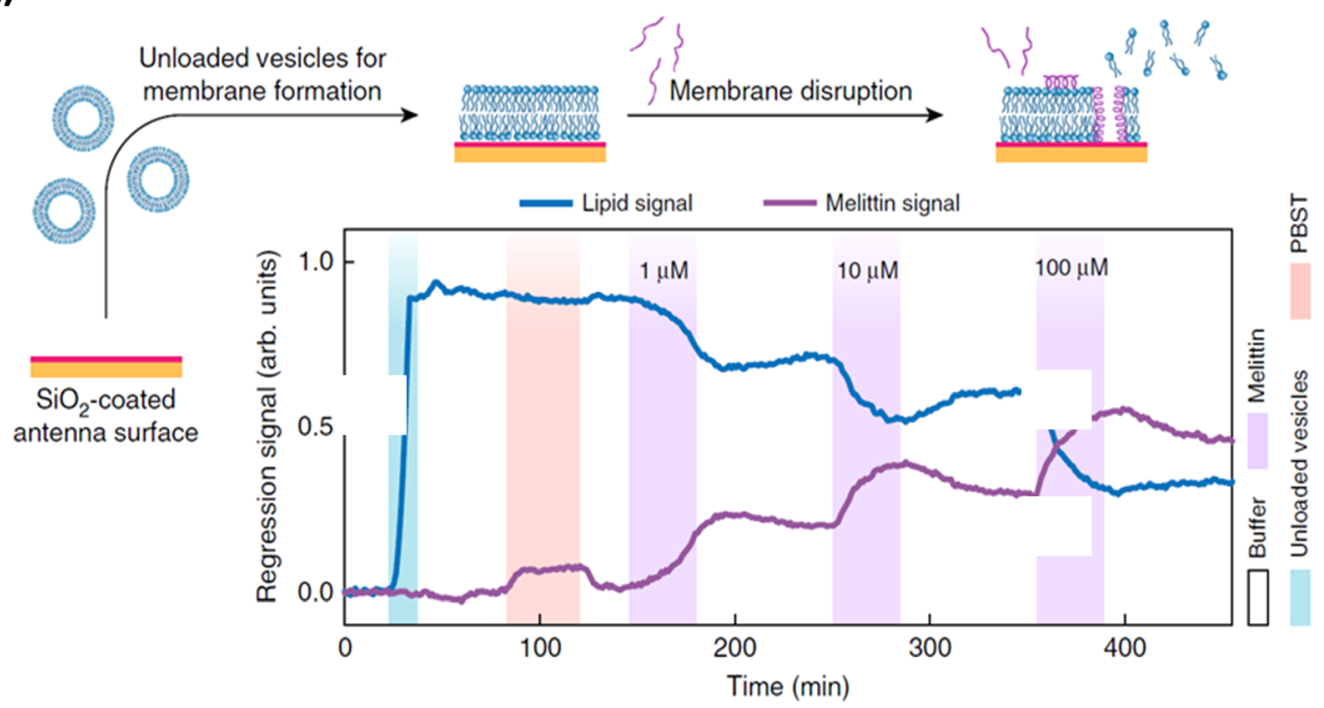

Figure 7: Cell studies and detection.

(a) The transmission spectra of the metasurface showing four peaks, which is produced by the electromagnetically induced transparency (EIT) behavior between adjacent ring structures. (b) Schematic of the metasurface. Each meta-atom consisted of five rings with diameters of 20, 28, 36,44 , and $52 \mu \mathrm{m}$ and the gap between each ring is $4 \mu \mathrm{m}$. (c) The frequency shift for different concentrations of epithelium-derived oral cancer cells (HSC3). (d) The frequency shift of the control oral cancer cells SCC4. (e) The frequency shift of SCC4 cancer cells treated with the chemotherapy drug, Cisplatin, within 24-96 h. (a)-(e) Reproduced with permission from Zhang et al., Appl. Phys. Lett. 108, 241105 (2016) [144]. Copyright 2016 AIP Publishing. (f) The metasurface structure of gold nanodipoles and simulated reflectance spectrum for the $\mathrm{CH}_{2}$ (green) and amide (red) band. (g) The presence of melittin (purple) results in a corresponding decrease in lipid (blue) signal, this trend becomes more prominent as the concentration in of melittin increases. (h) Linear regression signals of gamma-aminobutyric acid (GABA), melittin, and lipid over time. (f)-(h) Reproduced with permission from Rodrigo et al., Nat. Commun. 9, 2160 (2018) [145]. Licensed under a Creative Commons Attribution 4.0 International License. (i) An infrared metasurface biosensor for label-free, real-time lipid membranes monitoring. The metasurface was designed with a gold nanoantenna array on top of a calcium fluoride substrate. The size of the nanoantennas array was 1$1.75 \mu \mathrm{m}$ in length. (j) Streptavidin was injected into the metasurface and the surface-enhanced infrared absorption (SEIRA) signal was used to detect the molecular binding between streptavidin and the biotin group on DPPE lipids. (i) and (j) Reproduced with permission from Limaj et al., Nano Lett. 16, 1502-1508 (2016) [141]. Copyright 2016 American Chemical Society. 


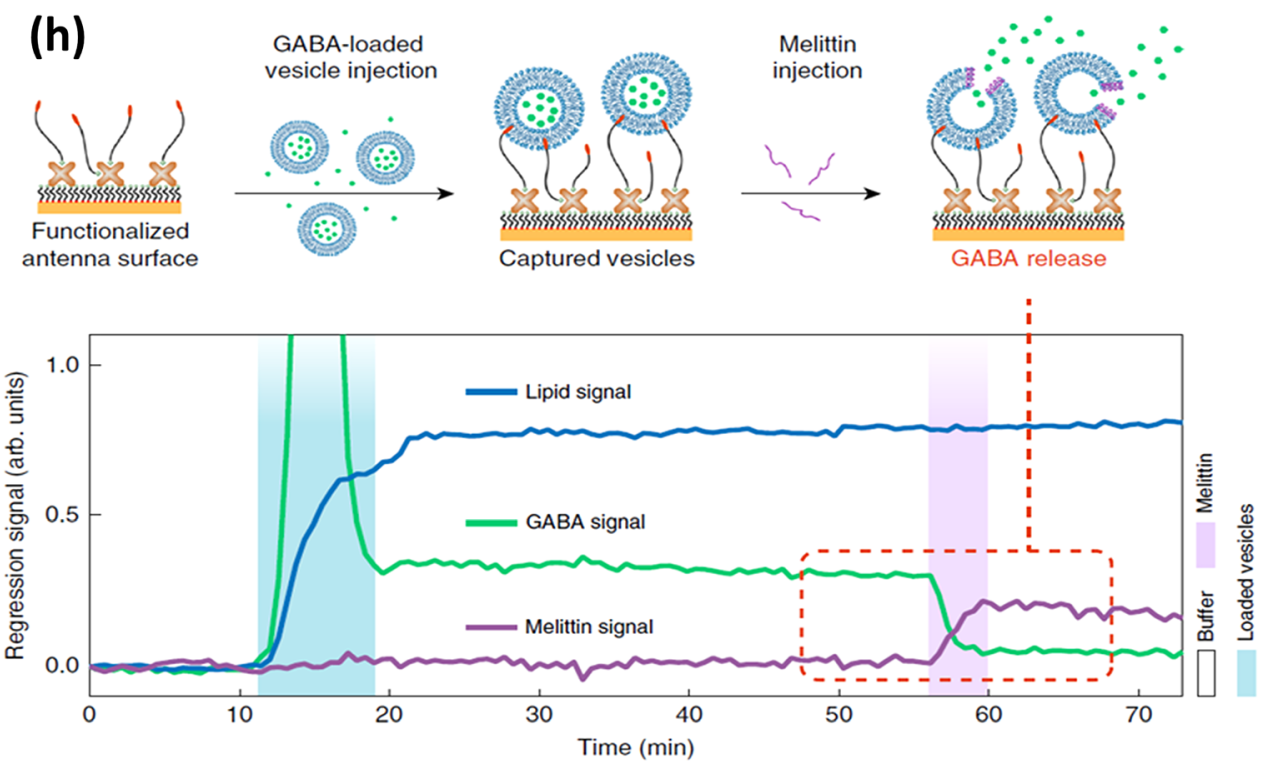

(i)

(j)
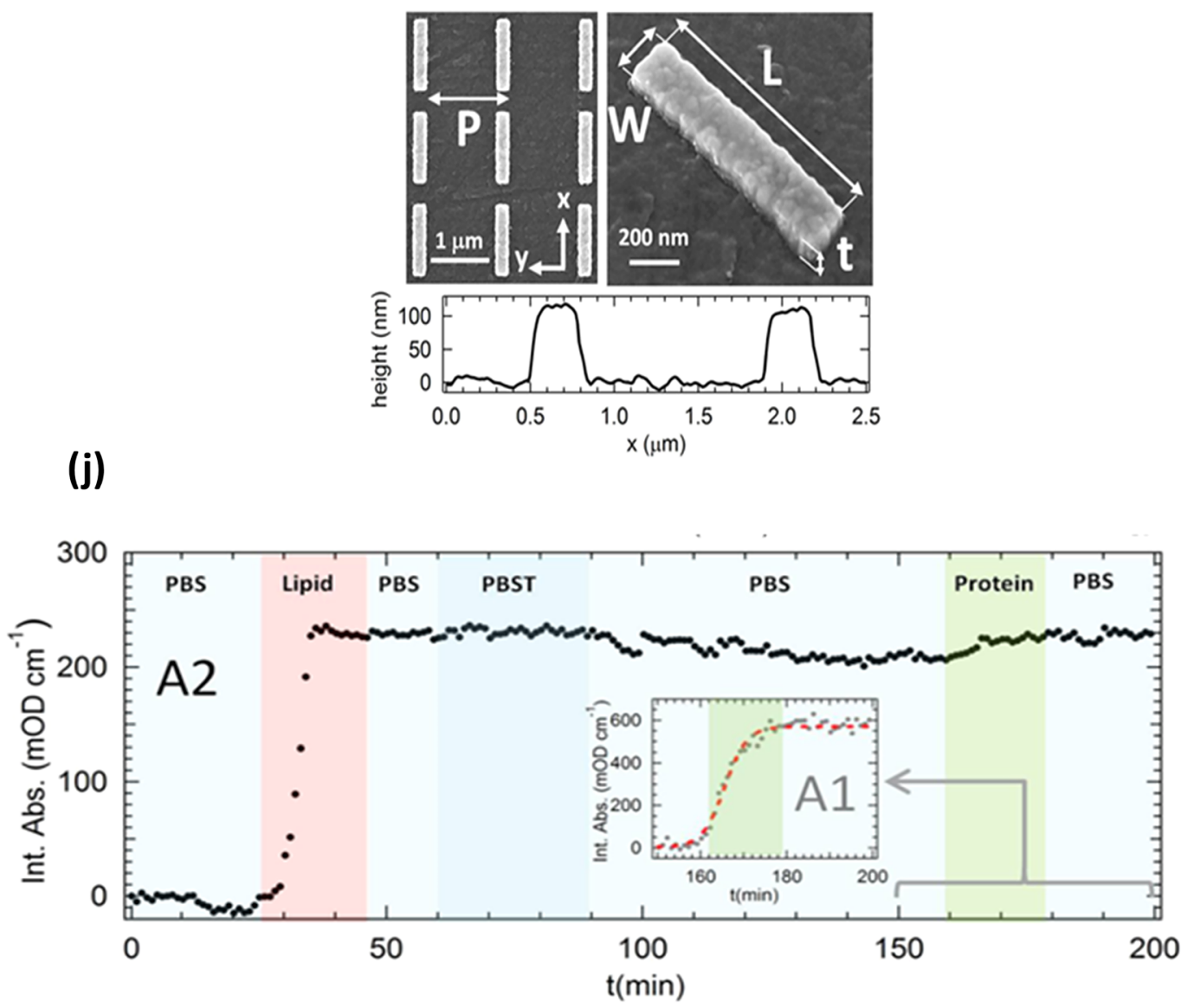

Figure 7: Continued. 
response shifted back to the initial reading after the treatment with a fungicide. This signal shift was due to refractive index induced capacitance change at the nanogap. Also, a specific antibody has been used for $E$. coli detection. The injection of $E$. coli resulted in a $23 \mathrm{GHz}$ shift in frequency.

\subsection{Cancer biomarkers}

Metasurface biosensors also find applications in cancer biomarker detection, including breast, liver, and oral cancer. An optofluidic metasurface biosensor has been developed for breast cancer biomarkers detection [146]. The metasurface was made of an array of silicon nanoposts that is constructed on a silicon on insulator substrate. Figure $8 \mathrm{a}$ and $\mathrm{b}$ show the schematic and the SEM image of the metasurface structure. The metasurface sensor performance was characterized with different concentrations of ethanol solutions ranged from 1.340 to 1.318 RIU and a sensitivity of $720 \mathrm{~nm} / \mathrm{RIU}$ was derived from the sensor response curve in refractometric sensing measurement (Figure $8 \mathrm{c}$ and d). It has further been demonstrated for the detection of an early-stage breast cancer biomarker, epidermal growth factor receptor 2 (ErbB2). Anti-ErbB2 antibodies were immobilized on the metasurface and the molecular binding with targeted ErbB2 molecules was recorded (Figure 8e). One can see a clear dose-response curve of resonance wavelength against antigen concentrations (Figure 8f) and the kinetic binding curve (Figure $8 \mathrm{~g}$ ) with a detection limit of $0.7 \mathrm{ng} / \mathrm{ml}$.

Another example is for liver cancer biomarker measurement [147]. The metasurface comprised of gold splitring resonators deposited on a silicon substrate (Figure $8 \mathrm{~h}$ and i). The inner radius was $24 \mu \mathrm{m}$ and the outer radius was $30 \mathrm{~nm}$ with a $6 \mathrm{~nm}$ width between the radii. The periodicity between each resonator was $90 \mu \mathrm{m}$. The performance of the metasurface was evaluated for the detection of two different liver cancer biomarkers, alpha-fetoprotein (AFP) and glutamine transferase isozymes II (GGT-II). Before the detection, the metasurface was functionalized with antibodies specific to each biomarker. Based on Figure 8j, it can be seen that the injection of AFP resulted in a resonance shift of $8.6 \mathrm{GHz}$ and based on Figure 8k, the injection of GGT-II resulted in a shift of $18.7 \mathrm{GHz}$. Another metasurface design based on a split ring resonator with double gaps that supports two resonance dips was proposed. The same experiment was repeated. Based on the results from Figure 81 and $\mathrm{m}$, the addition of the AFP resulted in a shift in resonance for both the dips and the same was observed with GGT-II injection.
Oral cancer cells (HSC3) cell apoptosis process was studied using electromagnetically induced transparency like metasurface [148]. The metasurface was designed with an array gold double split-ring resonator supported on a $25 \mu \mathrm{m}$ polyimide dielectric layer and deposited on a silicon substrate. The splits were asymmetric of each other as shown in Figure 9a and b. Transmission modeling was conducted for the analyte refractive index from 1 to 1.6 RIU (Figure 9c), which gives the sensor response curve of the metasurface (Figure 9d). It has further been applied for the studies of the relationship between an anti-cancer drug, cisplatin, and the cancer cell apoptosis process. Figure 9e and $\mathrm{f}$ show the measurement results for the different doses of cisplatin, while Figure $9 \mathrm{~g}$ and $\mathrm{h}$ indicate the cancer cells' response against time for the anticancer drug. Experimental results show that the metasurface provides a sensitivity of $900 \mathrm{kHz} /$ cell $\mathrm{ml}^{-1}$.

Beyond sensing, Vrba et al. [149] demonstrated that metasurfaces can also be used for hyperthermia cancer treatment. Three different metasurfaces were designed and tested using a muscle tissue model. The first metasurface consisted of four metamaterial units and was based on microcoplanar technology. It was made on an FR4 substrate and used a ground plane beneath the substrate to suppress the backside radiation. The capacitor consisted of 10 fingers of dimensions of 18,1 , and $1 \mathrm{~mm}$ in length, width, and gap. The same dimension was applied to the other two metasurfaces too. The second metasurface was based on the microstrip technology and consisted of four inductive strips extending from four fingers capacitors to the grounded capacitive plates. The third metasurface was also based on the microstrip; however, it consisted of two other layers, FR4 substrate and air. The metasurface was designed with five vertical inductive elements that run from the upper FR4 to the lower FR4 ground plane. The muscle tissue model used to test the metasurfaces was represented by a $1 \mathrm{~cm}$ layer of deionized water. The performance of the metasurface was determined by the reflection coefficient, current distribution, and power deposition value, while the optimized pattern covered $84 \%$ of the treatment volume with $25 \%$ the power deposition value.

\section{Future directions}

Metasurfaces have been demonstrated with applications in nonbiomedical fields with extraordinary features that may in the future be applied for bioimaging and biosensing areas as well. Firstly, metasurfaces-enabled adaptive 
(a)

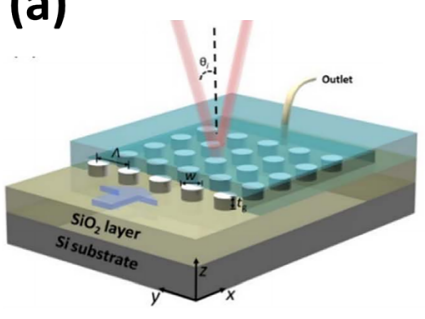

(e)

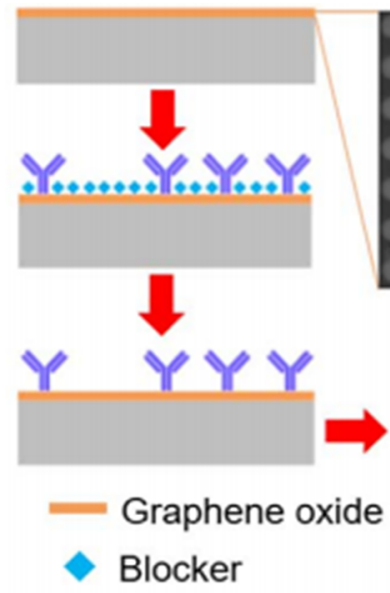

(b)

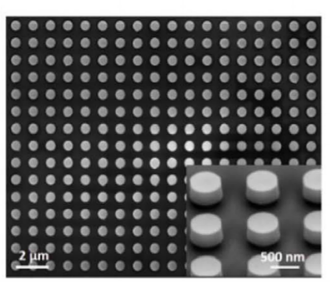

(c)

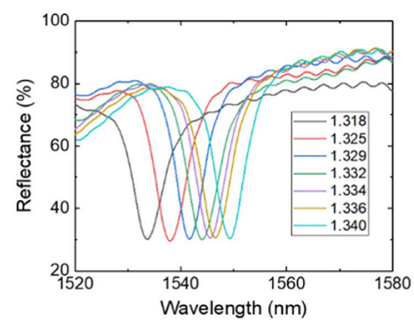

(f)
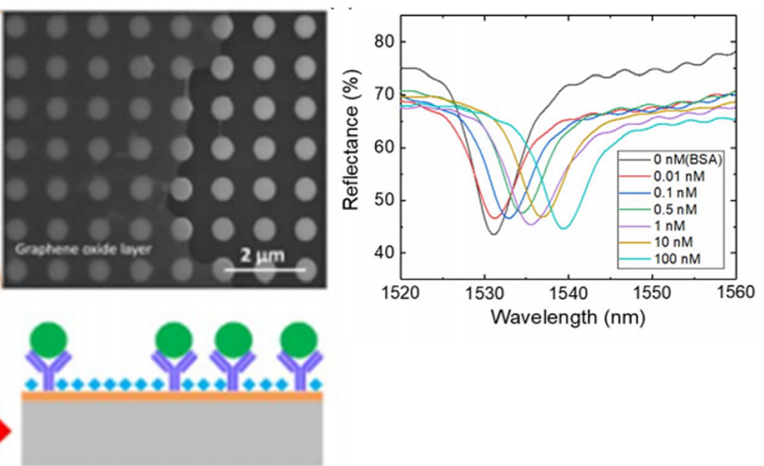

(d)

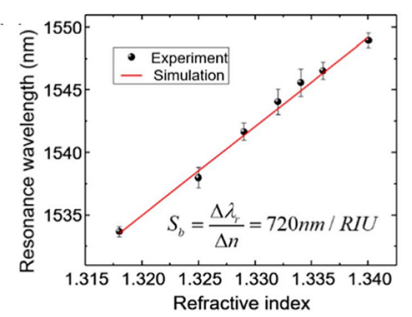

(g)

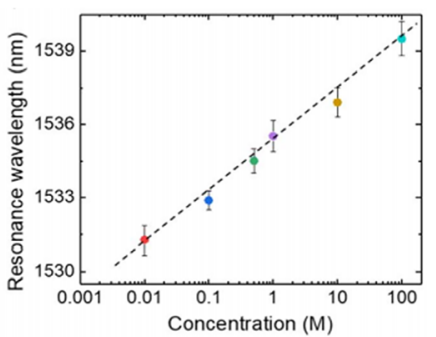

(h)
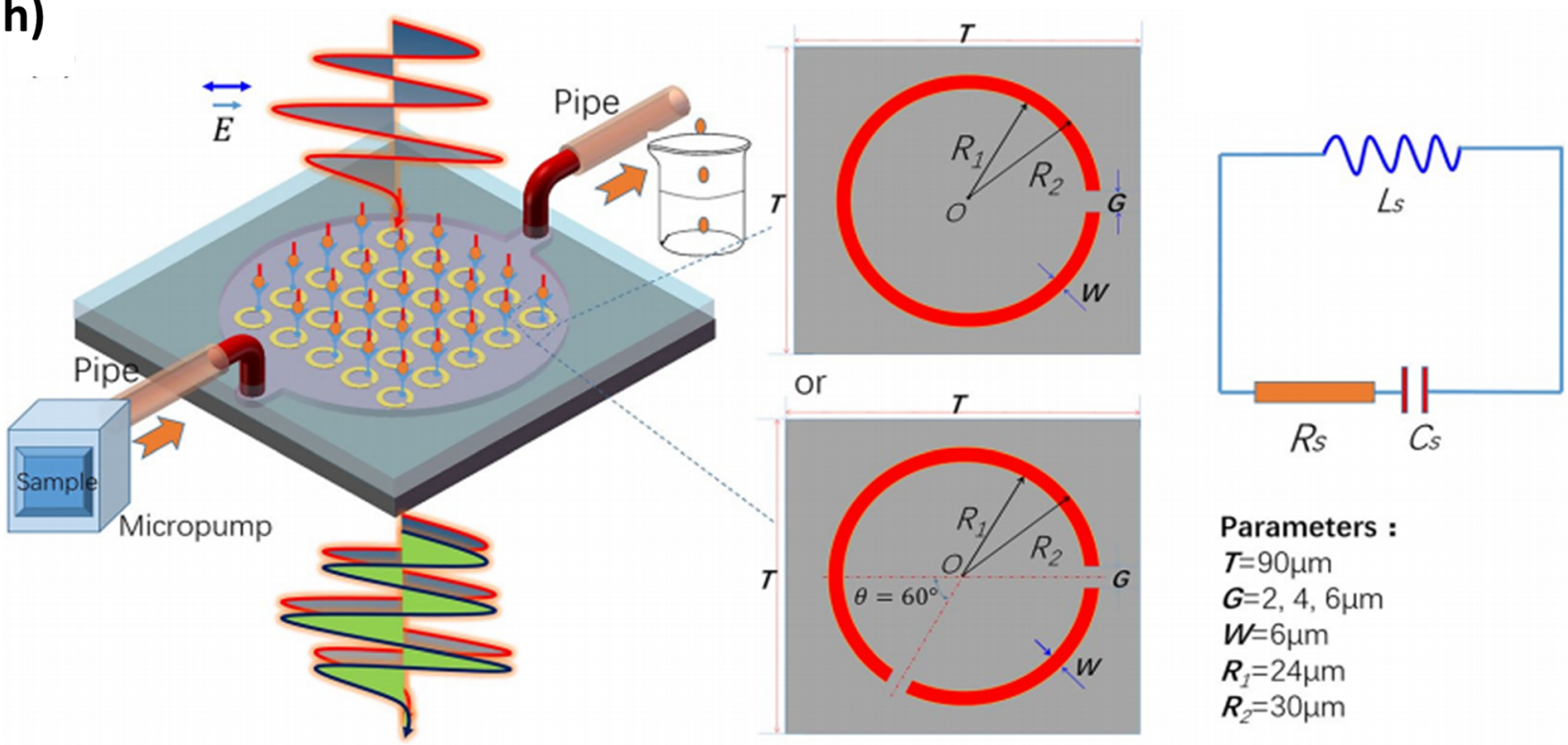

Parameters :

$T=90 \mu \mathrm{m}$

$\boldsymbol{G}=2,4,6 \mu \mathrm{m}$

$W=6 \mu \mathrm{m}$

$R_{1}=24 \mu \mathrm{m}$

$R_{2}=30 \mu \mathrm{m}$

Figure 8: Cancer biomarkers detection.

(a) Schematic of the metasurface with a silicon nanopost array. The sensor surface was integrated with a PDMS based optofluidic chamber for cancer biomarker detection. (b) SEM image of the nanopost array. (c) Measurement results for ethanol solutions with different refractive index values (1.318$1.340 \mathrm{RIU}$ ). (d) Experimental and simulated sensor response curve of the metasurface sensor. (e) The sensor surface was functionalized with antiErbB2 molecules for the detection of early-stage breast cancer protein biomarker, epidermal growth factor receptor 2 (ErbB2), by monitoring the plasmonic resonance wavelength shift. (f) The reflection spectra for different concentrations of ErbB2 protein (0.01-100 nM). (g) The dose-response curve for the ErbB2 protein. The detection limit was found to be $0.7 \mathrm{ng} / \mathrm{ml}$. (a)-(g) Reproduced with permission from Wang et al., Biosens. Bioelectron. 107, 224-229 (2018) [146]. Copyright 2018 Elsevier. (h) (Left) Schematic of the THz metasurface integrated with a microfluidic chip. (Right) Design of the meta-atom. (i) Transmission spectra of alpha-fetoprotein (AFP) with the single split-ring resonator. The black curve represents the metasurface without samples and the red curve represents the metasurface with biomolecules. (j) Transmission of GGT-II with the single split-ring resonator. (k) Transmission of AFP with the double split-ring resonator. (l) Transmission of GGT-II with the double split-ring resonator. (h)-(l) Reproduced with permission from Geng et al., Sci. Rep. 7, 16378 (2017) [147]. Licensed under a Creative Commons Attribution 4.0 International License. 
(i)
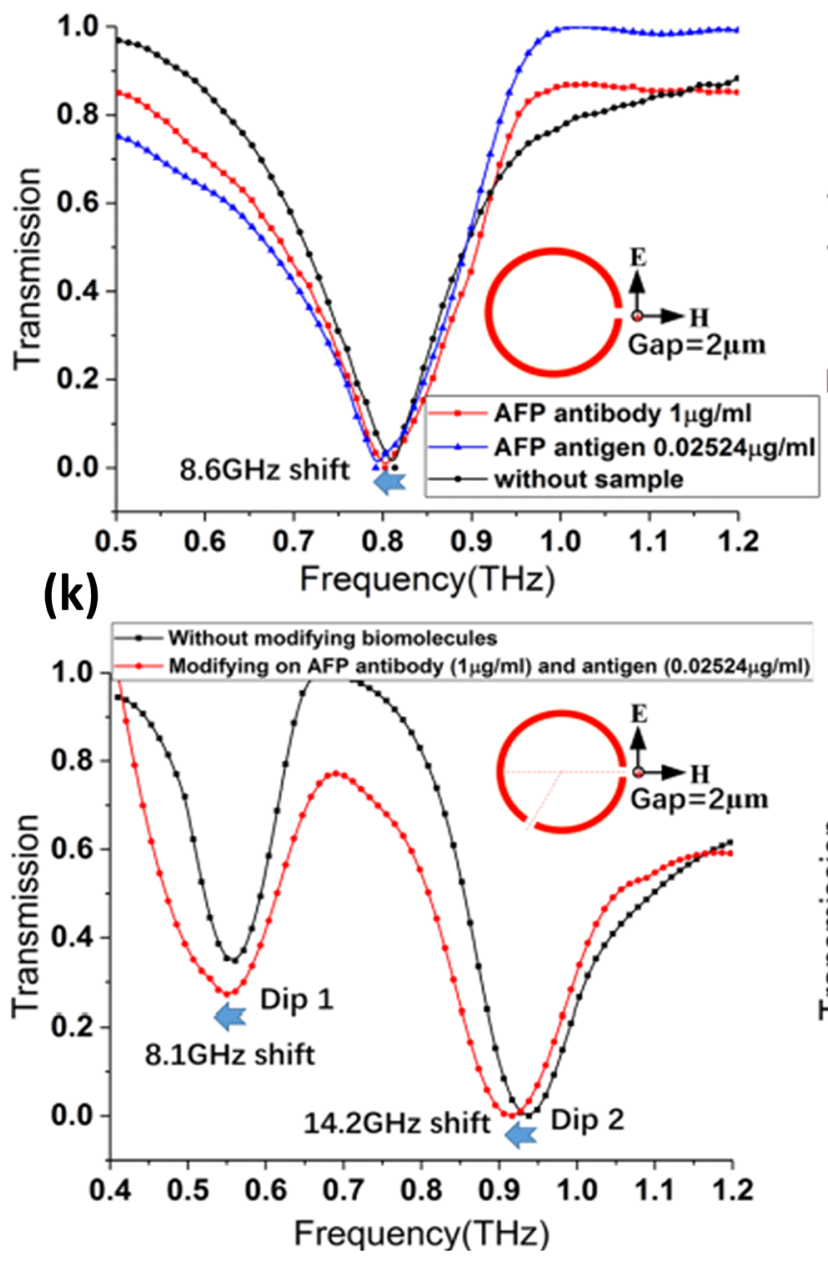

Figure 8: Continued.

optics (AO) devices can be applied in confocal microscopy, two-photon microscopy, and super-resolution imaging for imaging within highly scattering biological media, such as tissues [169-171]. Examples of such metasurfaces with AO features include: microelectromechanical systems (MEMS)-based focal length tuning [172] and coma (offaxis) aberration correction (Figure 10a) [80], soft materialbased focal length tuning by mechanical stretching $[65,67]$ and elastomer actuator-based focal length tuning, astigmatism and shift corrections by electrical deformation (Figure 10b) [66]. Secondly, metasurfaces-enabled optical fibers, which combine the light manipulation capabilities of the metasurface and the flexibility of fibers, can be applied for in vivo bioimaging and remote biosensing [125, 173]. For example, light focusing [87] at the fiber tip can be used for high-resolution in vivo imaging and surface
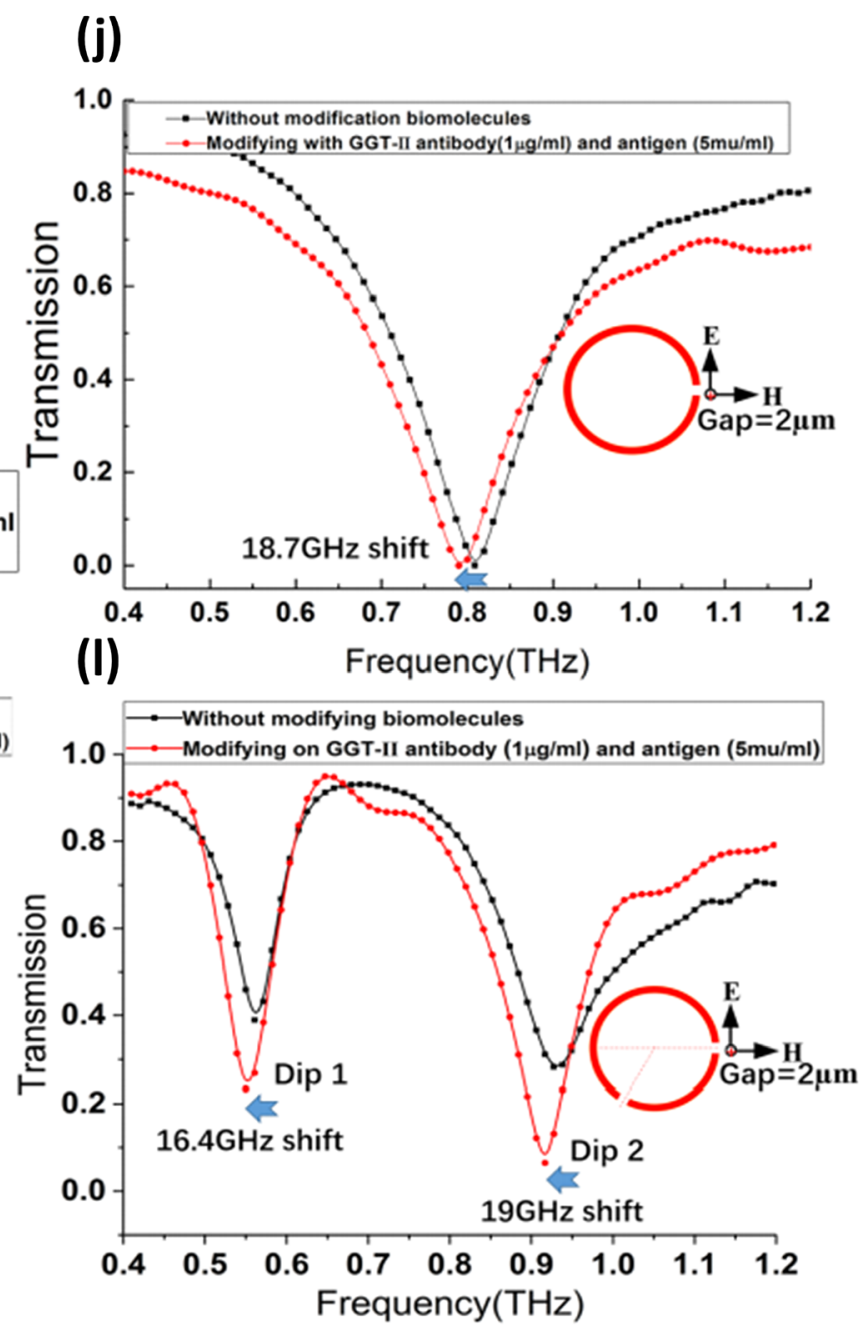

enhancement of the light field at the fiber tip can be used for biosensing $[86,174]$. Thirdly, an advantage of metalenses is the ease to generate structured light such as Laguerre-Gaussian and Bessel beams, etc. because the phase profile of a metasurface can be designed with subwavelength accuracy. Metasurface-based structured beams such as vortex beams $[175,176]$, Bessel beams (see Figure 10c) [177] and Bessel beams on a fiber tip [178]. This is important for STED microscopy [179], light-sheet microscopy [180], and two-photon microscopy [181]. Fourthly, conformal metasurfaces [64, 182] are another intriguing area of exploration. Metasurface devices can be fabricated on soft substrates to allow for flexibility, stretchability, foldability ("origami metasurfaces" [183]), and actuability, enabling novel form factors and tunable features. Conformal metasurfaces will be useful in smart wearable 
(a)

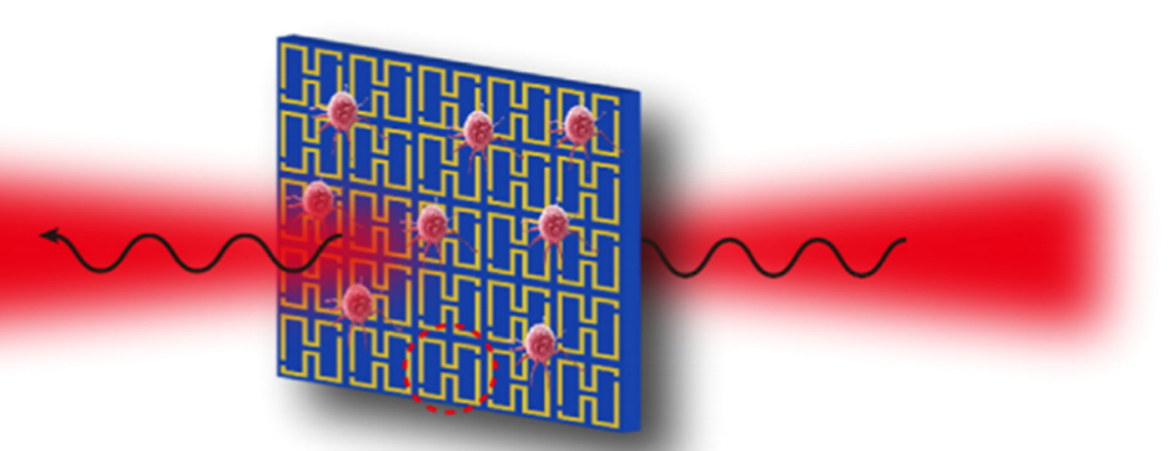

(b)

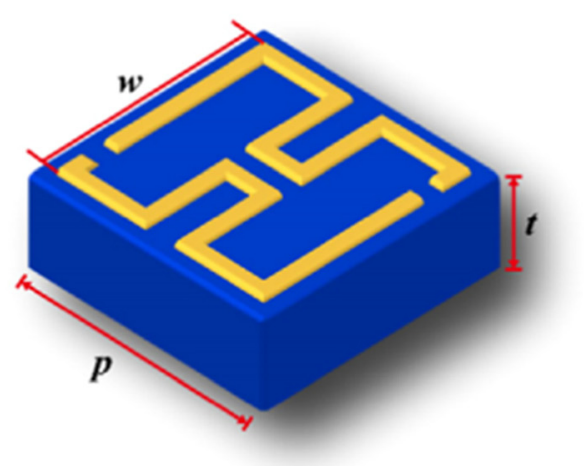

(c)

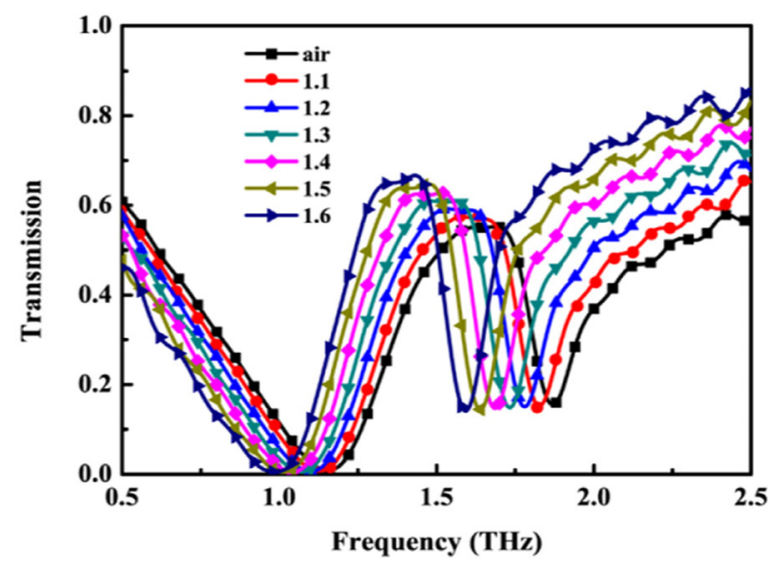

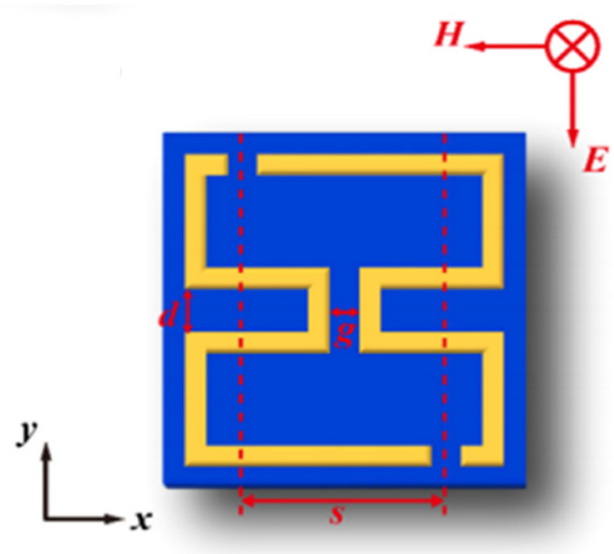

(d)

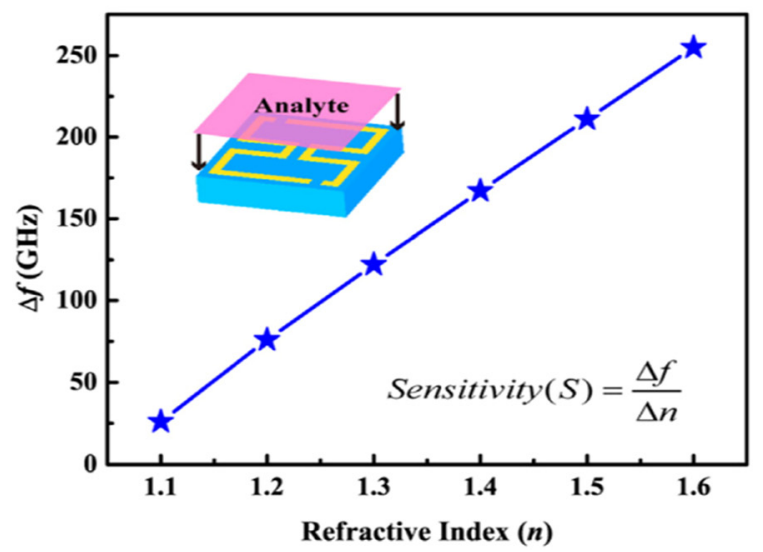

Figure 9: Oral cancer cells (HSC3) apoptosis detection with electromagnetically induced transparency like metasurface.

(a) Schematic of the electromagnetically induced transparency like (EIT-like) based metasurface. (b) Metasurface structure with symmetrybreaking double-splits ring resonators. (c) Modeling the transmission curve for different refractive index (1-1.6 RIU). (d) Frequency shift response curve for different refractive indices. (e) and (f) Measurement results for the different dosage of cisplatin. (g) and (h) Cancer cells response against time for the anti-cancer drug. Experimental results show that the metasurface provides a sensitivity of $900 \mathrm{kHz}^{-c e l l ~} \mathrm{ml}^{-1}$. (a)-(h) Reproduced with permission from Yan et al., Biosens. Bioelectron. 126, 485-492 (2019) [148]. Copyright 2019 Elsevier. 
(e)

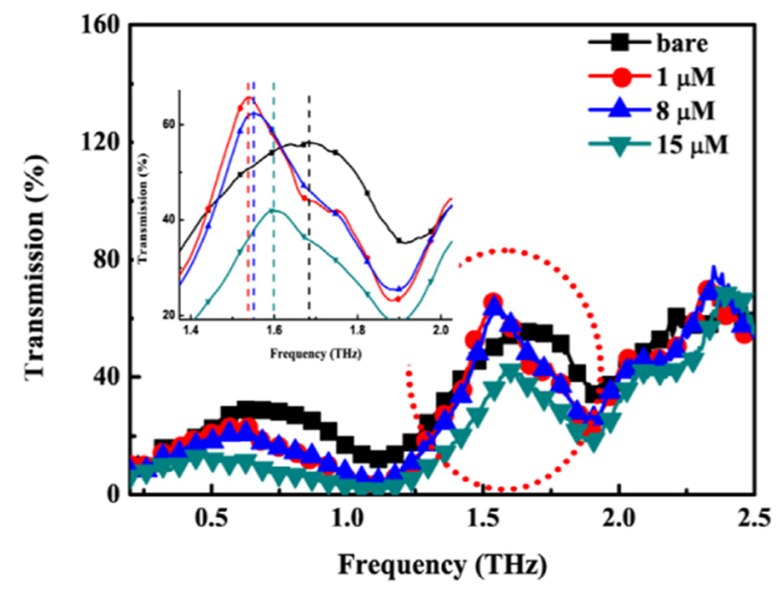

(g)

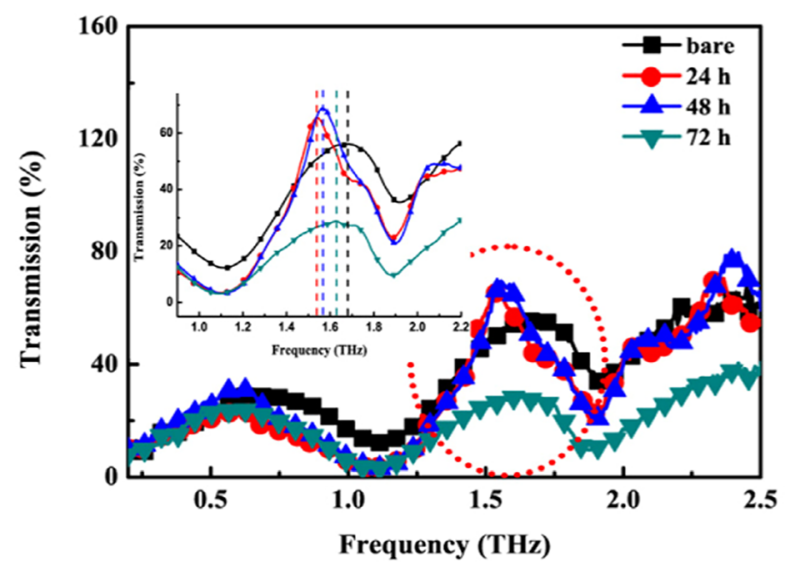

Figure 9: Continued.

and implantable health-care products such as optical bioimagers and biosensors that can be worn on the skin (see Figure 10d) or origami-folded structures directly injected and automatically unfurled inside the body. Lastly, metasurfaces-based optical tweezing and trapping can be applied in studies such as single-molecule biophysics, and cell-cell interactions. Tweezers create a 3D trap that requires tight focusing of light. Traditional microscope objectives are typically used to achieve such a high NA and tightly focused beam, but they are bulky and are often not compatible with lab-on-a-chip systems, where optical can be used to manipulate and analysis of cells and bacteria. Metasurfaces may create an entirely planar structure (f)

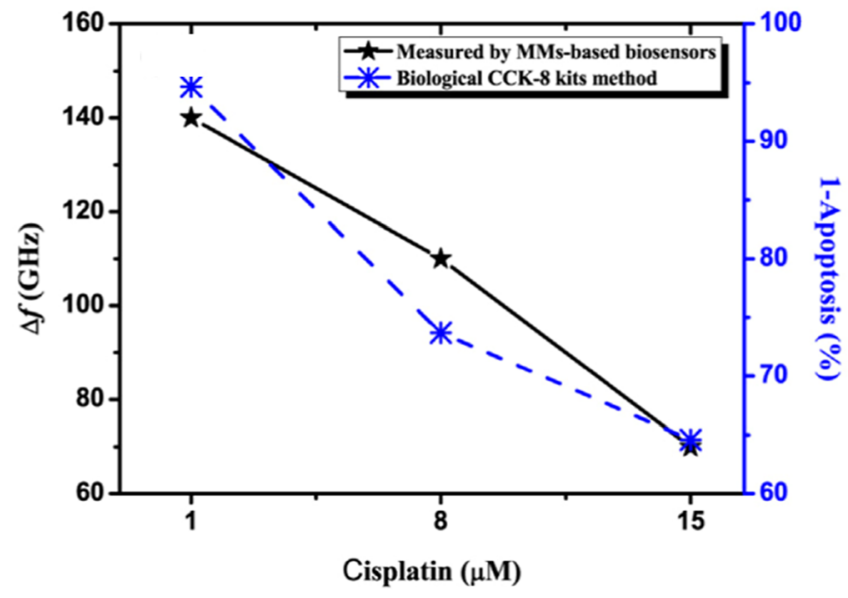

(h)

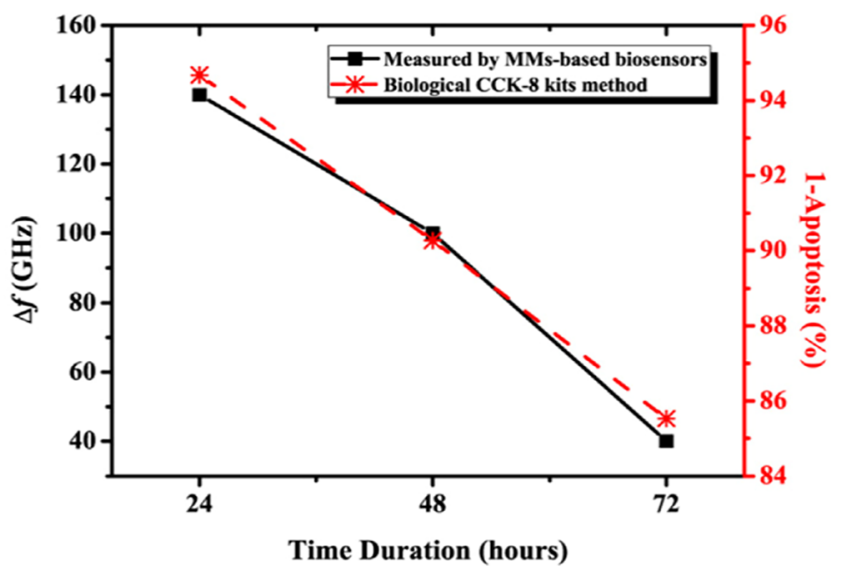

capable of focusing light from a collimated incident beam into a tight spot, and yield generating traps with structured beams. In recent work [184], planar metalenses created optical traps in a microfluidic environment. The trap stiffness was between 13

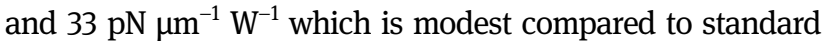
traps but can be improved with better metasurface design. An important facet of the work showed metalenses offer flexibility in the phase profile design and, thus, may lead far beyond the simple focusing of light. The authors then demonstrated the creation of a conical beam for enhanced trapping of objects.

Not only can metasurface devices be used for bioapplications but conversely the vast biological world itself can be an inspiration for the nanophotonics community. 
(a)

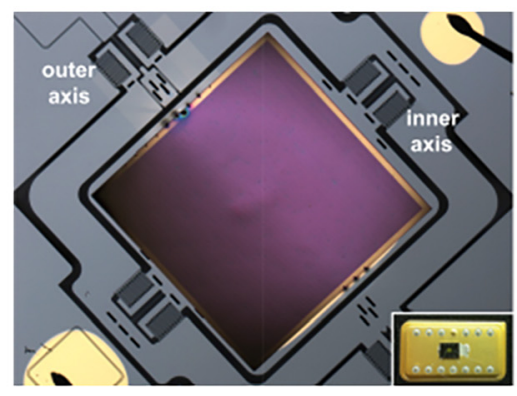

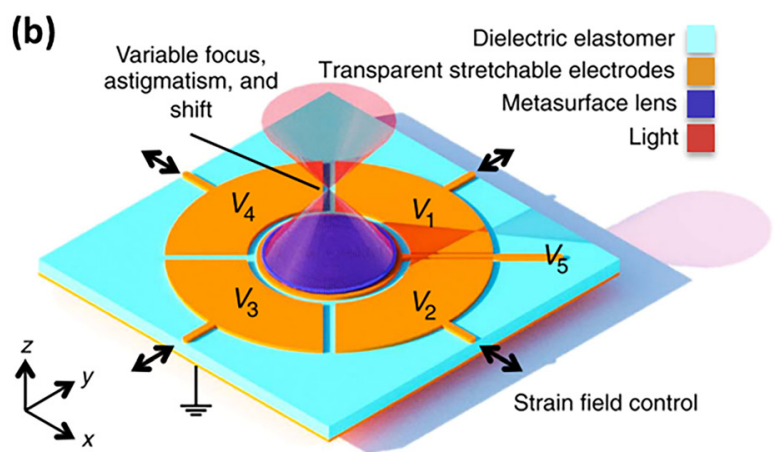

(c)
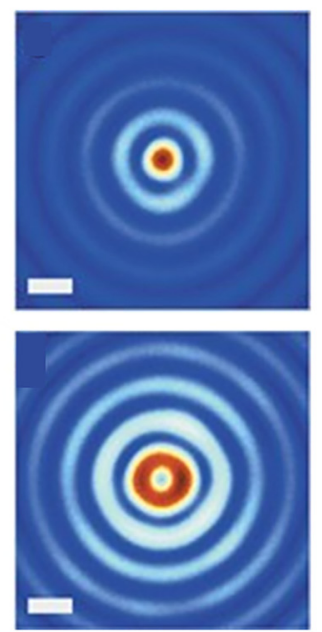

(d)

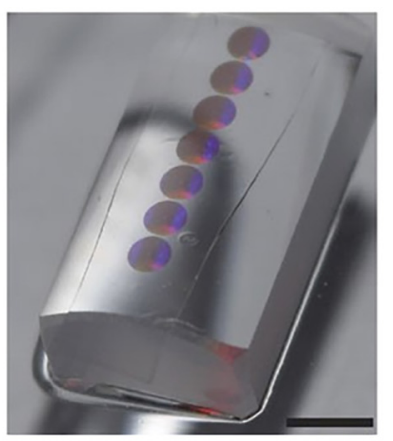

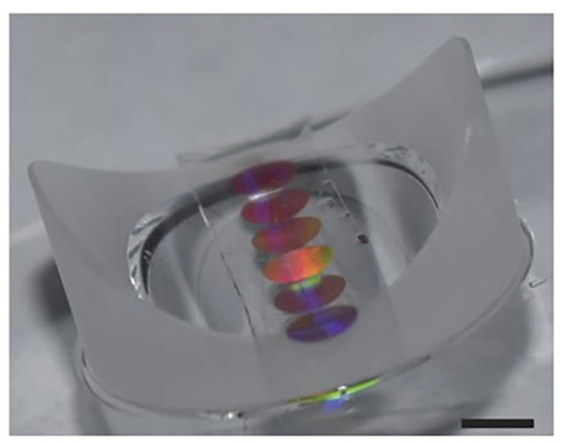

(e)

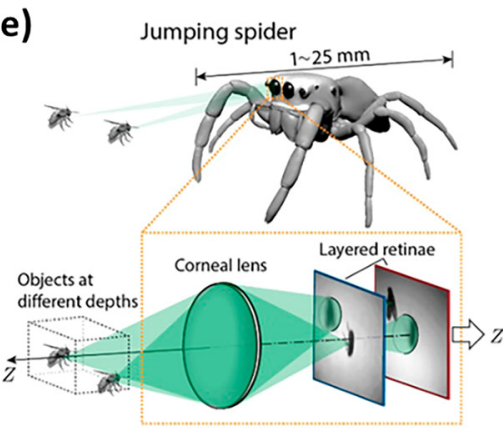

(f)

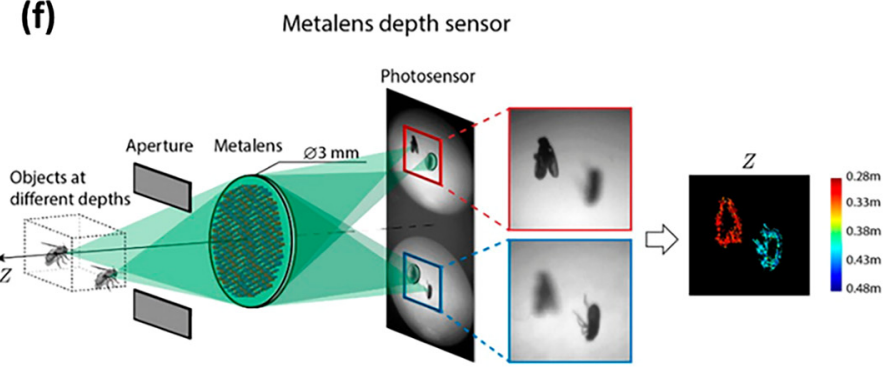

Figure 10: Future directions.

(a) Optical microscope image showing a metalens on a two-axis rotational microelectromechanical system (MEMS) which changes the relative angle between the incident light and the metalens. The inset is the integrated device with a dual in-line package. (a) Reproduced with permission from Roy et al., APL Photonics 3, 021302 (2018) [80]. Copyright 2018 Author(s). This article is distributed under a Creative Commons Attribution (CC BY) license. (b) Schematic showing an electrically tunable metasurface device with five controllable electrode configurations to correct for defocus, astigmatism, and shift aberrations. (b) Reproduced with permission from She et al., Sci. Adv. 4 , eaap9957 (2018) [66]. Distributed under a Creative Commons Attribution-NonCommercial license. (c) Measured intensity profiles of two Bessel beams with numerical aperture (NA) $=0.7$ at $\lambda=575 \mathrm{~nm}$. Scale bar, $500 \mathrm{~nm}$. (c) Adapted with permission from Chen et al., Light Sci. Appl. 6, e16259-e16259 (2017) [177]. Licensed under a Creative Commons Attribution-NonCommercial-ShareAlike 4.0 International License. (d) Conformal metasurface on a convex (left) and a concave glass cylinder (right). In both cases, the metasurfaces make cylinders behave like converging aspherical lenses. Scale bar, 2 mm. Reproduced with permission from Kamali et al., Nat. Commun. 7, 11618 (2016) [64]. Licensed under a Creative Commons Attribution 4.0 International License. (e) Depth perception of a jumping spider is achieved with the multitiered retina structure of each principal eye which captures multiple images of the same scene with different amounts of defocus. (f) Depth perception of a metalens depth sensor is achieved by a metalens that captures two images of the same scene with different amounts of defocus. The metalens was constructed by spatially multiplexing two phase profiles that focus on the photosensor with a lateral offset and different focal lengths. The depth map was generated by a depth reconstruction algorithm. (e) and (f) Reproduced with permission from Guo et al., Proc. Natl. Acad. Sci. 116, 22959-22965 (2019) [188]. Copyright 2019 the Author(s). This article is distributed under Creative Commons Attribution License 4.0 (CC BY). 
Learning from animals with evolutionary advantages gifted by Nature has led to the development of many biomimetic metasurface devices. For example, Yi et al. [185] observed that sensing the direction of sounds is a special capability of certain small animals which relies on a coherent coupling of soundwaves between the two ears. Inspired by this observation, they developed a metasurface-based ultrasensitive photodetector that is able to detect the incident angle of light with an angular sensitivity of $0.32^{\circ}$. The second example is by Shi et al. [186] who studied the silver ants living in the Saharan desert and developed an idea of a biomimetic metasurface-based coating for the passive effective cooling of objects. The third example is by Basiri et al. [187] who designed a metasurface-based polarization filter device inspired by the polarization sensing of the compound eyes of mantis shrimps. The peak extinction ratio of the metasurface device could reach a value of 35 , and transmission efficiency was close to $80 \%$ in the near-infrared wavelength region. Another example is the development of a metasurfacebased depth sensor inspired by the eyes of jumping spiders. Guo and Shi led the research and mimicked the depth perception mechanism of jumping spiders and developed a metalens equivalent depth sensor which was compact (3 $\mathrm{mm}$ in diameter for a depth measurement range of $10 \mathrm{~cm}$ ), single-shot and required a small amount of computation (fewer than 700 floating-point operations per output pixel with microwatts energy) [188]. Figure 10e and $f$ show the working principles of the depth perception of a jumping spider and a metalens equivalent depth sensor.

Although there are many potential future directions to explore, challenges still exist that need to be addressed. Firstly, metalenses generally suffer more significant chromatic aberration than a conventional curved lens. For bioapplications that require broadband wavelength excitation and focusing or illumination at one wavelength and collection at another wavelength far from that (e.g. multiphoton imaging), large-area metalenses (a lens diameter on the order of centimeter) with achromatic focusing is a technical challenge. In fact, this is a hot research topic in the entire metasurface community. Recently, demonstrations of largearea metalenses with single wavelength operation in the visible and near-infrared wavelength ranges $[10,14,83,189]$ and achromatic focusing of small area metalenses $[190,191]$ have been reported. Hence, it is promising that this challenge can be solved soon with the advancement in both metasurface design methodologies and fabrication techniques [44]. Secondly, most metasurfaces operate with an incidence light that is normal to the interface, which translates to a limited FOV. Incident light that comes at an angle will result in coma aberration. For conventional optical components, an increased FOV can be realized with a stack of lenses, i.e., a lens assembly. Similarly, for metalenses, FOV may be increased by adopting a multilayered structure [192] with advanced design methodologies such as the inverse design with topological optimization [193]. Thirdly, challenges on clinical translation of metasurfaceempowered biomedical devices need to be addressed. Factors such as cost, reliability, and reproducibility must be taken into account at the early stage of the device development journey. The studies by Wilson et al. [194] and Popp et al. [195] review the challenges of transforming a laboratory prototype to a viable clinical product such as conducting preclinical and clinical trials and obtaining regulatory approval. Among these, the most important lesson is to constantly get feedback from the clinicians on the device design and performance to make sure that the device addresses the unmet clinical needs for a smooth clinical translation in the future.

\section{Conclusions}

In this review, we have covered the use of metasurfaces for bioimaging and biosensing applications. A variety of metasurface-based bioimaging techniques have been developed, spurred by the advantages of uniquely tailored imaging features and small device footprints. In particular, among these, we have described the latest developments in metasurface-based chiral imaging, OCT endoscopy imaging, fluorescent imaging, two-photon fluorescent microscopy, PSIM super-resolution imaging, MRI imaging, and QPI imaging. In addition, metasurfaces for biosensing applications are also reviewed, in which fully customizable nanopatterning and intimate interaction of these extremely thin devices are leveraged to measure biological signals in novel ways. For example, these include the detection of antibody and protein binding using metasurface-based hyperspectral imaging; detection of DNAs using graphene metasurfacebased surface-enhanced fluorescence spectroscopy; detection of in situ cell apoptosis using a metasurface; detection of cancer biomarkers in the liver and breast using metasurfaces combined with optofluidics and graphene oxide.

It is the authors' view that going forward, the future of the metasurface-based bioimaging and biosensing provides several different exciting paths of exploration. In vivo optical imaging is a potential area, where metalenses hold great future promises. In this area, two- or multiphoton imaging and endoscopy are areas of promise for the application of metalenses. Typically, the two-photon imaging systems are 
complex and bulky, which are major impediments to their practical use, especially in the consideration of animal geometries and animal motion. Another in vivo imaging technique is endoscopy which must be engineered while subject to severe space constraints. The conventional micro-optics necessitated by these constraints provide for limited capabilities, which palein comparison to that which can be built by large, external systems. Metalenses offer interesting engineering possibilities to enable system complexity and extreme compactification, opening up possibilities for working smoothly with living bodies instead of around them. Last but not least, an emerging trend in biophotonics is multimodality: the collection of more than one form of information by light or other methods. For example, this may involve combining morphological information with molecular information. Such a combination of data from a variety of imaging techniques leads to better sensitivity and specificity which in turn means a more informed decision may be made that can directly improve medical diagnosis. The inherent compactness of metasurface devices lends itself to the possibility of integrating multiple devices into an ultrasmall size.

As metasurfaces biomedical devices become better explored, we envision a future where imaging, sensing, and perhaps even treatment in health care can be done with extremely small and flat metasurface devices that close the gap in the interface with living systems while at the same time be significantly less intrusive and noticeable.

Acknowledgments: The research is supported by the Agency of Science, Technology and Research (A*STAR), under its Industry alignment fund prepositioning program, Award H19H6a0025. K. D. thanks the UK Engineering and Physical Sciences Research Council through grant EP/ P030017/1. S. Z. thanks Dr. Alan J. She for the helpful discussions and editing of the manuscript.

Author contribution: All the authors have accepted responsibility for the entire content of this submitted manuscript and approved submission.

Research funding: The research is supported by the Agency of Science, Technology and Research (A*STAR), under its Industry alignment fund prepositioning program, Award H19H6a0025. K. D. thanks the UK Engineering and Physical Sciences Research Council through grant EP/P030017/1.

Conflict of interest statement: The authors declare no conflicts of interest regarding this article.

\section{References}

[1] V. G. Veselago, "Experimental demonstration of negative index of refraction,” Sov. Phys. Usp., vol. 10, p. 509, 1968.
[2] J. B. Pendry, A. J. Holden, W. J. Stewart, and I. Youngs, "Extremely low frequency plasmons in metallic mesostructures," Phys. Rev. Lett., vol. 76, pp. 4773-4776, 1996.

[3] D. R. Smith, W. J. Padilla, D. C. Vier, S. C. Nemat-Nasser, and S. Schultz, "Composite medium with simultaneously negative permeability and permittivity," Phys. Rev. Lett., vol. 84, pp. 4184-4187, 2000.

[4] N. I. Zheludev and Y. S. Kivshar, "From metamaterials to metadevices,” Nat. Mater., vol. 11, pp. 917-924, 2012.

[5] W. Cai, U. K. Chettiar, A. V. Kildishev, and V. M. Shalaev, "Optical cloaking with metamaterials," Nat. Photonics, vol. 1, p. 224, 2007.

[6] J. B. Pendry, D. Schurig, and D. R. Smith, "Controlling electromagnetic fields,” Science (80-), vol. 312, pp. 1780-1782, 2006.

[7] C. M. Soukoulis and M. Wegener, "Optical metamaterials-more bulky and less lossy," Science (80-), vol. 330, pp. 1633-1634, 2010.

[8] N. Yu, P. Genevet, M. a Kats, et al., "Light propagation with phase discontinuities: generalized laws of reflection and refraction," Science (80-), vol. 334, pp. 333-337, 2011.

[9] S. Zhang, M.-H. Kim, F. Aieta, et al., "High efficiency near diffraction-limited mid-infrared flat lenses based on metasurface reflectarrays," Opt. Express, vol. 24, p. 18024, 2016.

[10] A. She, S. Zhang, S. Shian, D. R. Clarke, and F. Capasso, "Large area metalenses: design, characterization, and mass manufacturing," Opt. Express, vol. 26, p. 1573, 2018.

[11] B. Groever, W. T. Chen, and F. Capasso, "Meta-lens doublet in the visible region,” Nano Lett., vol. 17, pp. 4902-4907, 2017.

[12] M. Khorasaninejad, W. T. Chen, R. C. Devlin, J. Oh, A. Y. Zhu, and F. Capasso, "Metalenses at visible wavelengths: diffractionlimited focusing and subwavelength resolution imaging," Science (80-), vol. 352, pp. 1190-1194, 2016.

[13] M. Khorasaninejad, F. Aieta, P. Kanhaiya, et al., "Achromatic metasurface lens at telecommunication wavelengths," Nano Lett., vol. 15, pp. 5358-5362, 2015.

[14] J.-S. Park, S. Zhang, A. She, et al., "All-glass, large metalens at visible wavelength using deep-ultraviolet projection lithography," Nano Lett., vol. 19, pp. 8673-8682, 2019.

[15] A. Arbabi, Y. Horie, A. J. Ball, M. Bagheri, and A. Faraon, "Subwavelength-thick lenses with high numerical apertures and large efficiency based on high-contrast transmitarrays," Nat. Commun., vol. 6, p. 7069, 2015.

[16] F. Aieta, P. Genevet, M. A. Kats, et al., "Aberration-free ultrathin flat lenses and axicons at telecom wavelengths based on plasmonic metasurfaces," Nano Lett., vol. 12, pp. 4932-4936, 2012.

[17] S. J. Byrnes, A. Lenef, F. Aieta, and F. Capasso, "Designing large, high-efficiency, high-numerical-aperture, transmissive meta-lenses for visible light," Opt. Express, vol. 24, pp. 1-16, 2015.

[18] M. Khorasaninejad, A. Y. Zhu, C. Roques-Carmes, et al., "Polarization-insensitive metalenses at visible wavelengths," Nano Lett., vol. 16, pp. 7229-7234, 2016.

[19] M. Khorasaninejad, Z. Shi, A. Y. Zhu, et al., "Achromatic metalens over $60 \mathrm{~nm}$ bandwidth in the visible and metalens with reverse chromatic dispersion," Nano Lett., vol. 17, pp. 1819-1824, 2017.

[20] Z. Ma, S. M. Hanham, Y. Gong, and M. Hong, "All-dielectric reflective half-wave plate metasurface based on the anisotropic 
excitation of electric and magnetic dipole resonances," Opt. Lett., vol. 43, p. 911, 2018.

[21] J. Hu, X. Zhao, Y. Lin, et al., "All-dielectric metasurface circular dichroism waveplate," Sci. Rep., vol. 7, p. 41893, 2017.

[22] E. O. Owiti, H. Yang, P. Liu, C. F. Ominde, and X. Sun, "Highly efficient birefringent quarter-wave plate based on all-dielectric metasurface and graphene," Opt. Commun., vol. 419, pp. 114-119, 2018.

[23] Y. Dong, Z. Xu, N. Li, et al., "Si metasurface half-wave plates demonstrated on a 12-inch CMOS platform," Nanophotonics, vol. 9, pp. 149-157, 2020.

[24] J. P. B. Mueller, K. Leosson, and F. Capasso, "Ultracompact metasurface in-line polarimeter," Optica, vol. 3, pp. 42-47, 2016.

[25] Q. Jiang, B. Du, M. Jiang, et al., "Ultrathin circular polarimeter based on chiral plasmonic metasurface and monolayer MoSe2," Nanoscale, vol. 12, pp. 5906-5913, 2020.

[26] E. Arbabi, S. M. Kamali, A. Arbabi, and A. Faraon, "Full-Stokes imaging polarimetry using dielectric metasurfaces," ACS Photonics, vol. 5, pp. 3132-3140, 2018.

[27] X. Ni, A. V Kildishev, and V. M. Shalaev, "Metasurface holograms for visible light,” Nat. Commun., vol. 4, p. 2807, 2013.

[28] G. Zheng, H. Mühlenbernd, M. Kenney, G. Li, T. Zentgraf, and S. Zhang, "Metasurface holograms reaching $80 \%$ efficiency," Nat. Nanotechnol., vol. 10, pp. 308-312, 2015.

[29] X. Zhang, S. Yang, W. Yue, et al., "Direct polarization measurement using a multiplexed Pancharatnam-Berry metahologram," Optica, vol. 6, p. 1190, 2019.

[30] W. T. Chen, A. Y. Zhu, M. Khorasaninejad, Z. Shi, V. Sanjeev, and F. Capasso, "Immersion meta-lenses at visible wavelengths for nanoscale imaging," Nano Lett., vol. 17, pp. 3188-3194, 2017.

[31] S. Chang, X. Guo, and X. Ni, "Optical metasurfaces: progress and applications,” Annu. Rev. Mater. Res., vol. 48, pp. 279-302, 2018.

[32] H.-T. Chen, A. J. Taylor, and N. Yu, "A review of metasurfaces: physics and applications,” Rep. Prog. Phys., vol. 79, p. 076401, 2016.

[33] N. Meinzer, W. L. Barnes, and I. R. Hooper, "Plasmonic metaatoms and metasurfaces," Nat. Photonics, vol. 8, pp. 889-898, 2014.

[34] Nanfang Yu, P. Genevet, F. Aieta, et al., "Flat optics: controlling wavefronts with optical antenna metasurfaces," IEEEJ. Sel. Top. Quantum Electron., vol. 19, p. 4700423, 2013.

[35] A. Y. Zhu, A. I. Kuznetsov, B. Luk'yanchuk, N. Engheta, and P. Genevet, "Traditional and emerging materials for optical metasurfaces,” Nanophotonics, vol. 6, pp. 452-471, 2017.

[36] G. Li, S. Zhang, and T. Zentgraf, "Nonlinear photonic metasurfaces," Nat. Rev. Mater., vol. 2, p. 17010, 2017.

[37] A. E. Minovich, A. E. Miroshnichenko, A. Y. Bykov, T. V. Murzina, D. N. Neshev, and Y. S. Kivshar, "Functional and nonlinear optical metasurfaces,” Laser Photon. Rev., vol. 9, pp. 195-213, 2015.

[38] A. V. Kildishev, A. Boltasseva, and V. M. Shalaev, "Planar photonics with metasurfaces," Science (80-), vol. 339, p. 1232009, 2013.

[39] S. Walia, C. M. Shah, P. Gutruf, et al., "Flexible metasurfaces and metamaterials: a review of materials and fabrication processes at micro- and nano-scales,” Appl. Phys. Rev., vol. 2, p. 011303, 2015.

[40] M. Song, D. Wang, S. Peana, et al., "Colors with plasmonic nanostructures: a full-spectrum review," Appl. Phys. Rev., vol. 6, p. 041308, 2019.
[41] C. U. Hail, A. U. Michel, D. Poulikakos, and H. Eghlidi, "Optical metasurfaces: evolving from passive to adaptive," Adv. Opt. Mater., vol. 7, p. 1801786, 2019.

[42] H. Liang, A. Martins, B.-H. V. Borges, et al., "High performance metalenses: numerical aperture, aberrations, chromaticity, and trade-offs," Optica, vol. 6, p. 1461, 2019.

[43] F. Ding, Y. Yang, R. A. Deshpande, and S. I. Bozhevolnyi, “A review of gap-surface plasmon metasurfaces: fundamentals and applications," Nanophotonics, vol. 7, pp. 1129-1156, 2018.

[44] W. T. Chen, A. Y. Zhu, and F. Capasso, "Flat optics with dispersion-engineered metasurfaces," Nat. Rev. Mater., vol. 5, pp. 604-620, 2020.

[45] F. Ding, A. Pors, and S. I. Bozhevolnyi, "Gradient metasurfaces: a review of fundamentals and applications," Rep. Prog. Phys., vol. 81, p. 026401, 2018.

[46] H.-H. Hsiao, C. H. Chu, and D. P. Tsai, "Fundamentals and applications of metasurfaces," Small Methods, vol. 1, p. 1600064, 2017.

[47] V.-C. Su, C. H. Chu, G. Sun, and D. P. Tsai, "Advances in optical metasurfaces: fabrication and applications [Invited]," Opt. Express, vol. 26, p. 13148, 2018.

[48] M. Khorasaninejad and F. Capasso, "Metalenses: versatile multifunctional photonic components," Science (80-), vol. 358, p. eaam8100, 2017.

[49] P. Genevet, F. Capasso, F. Aieta, M. Khorasaninejad, and R. Devlin, "Recent advances in planar optics: from plasmonic to dielectric metasurfaces," Optica, vol. 4, p. 139, 2017.

[50] S. Jahani, and Z. Jacob, "All-dielectric metamaterials," Nat. Nanotechnol., vol. 11, pp. 23-36, 2016.

[51] A. I. Kuznetsov, A. E. Miroshnichenko, M. L. Brongersma, Y. S. Kivshar, and B. Luk'yanchuk, "Optically resonant dielectric nanostructures," Science (80-), vol. 354, p. aag2472, 2016

[52] A. Tittl, A. John-Herpin, A. Leitis, E. R. Arvelo, and H. Altug, "Metasurface-based molecular biosensing aided by artificial intelligence," Angew. Chem. Int. Ed., vol. 58, pp. 14810-14822, 2019.

[53] D. Lee, J. Gwak, T. Badloe, S. Palomba, and J. Rho, "Metasurfaces-based imaging and applications: from miniaturized optical components to functional imaging platforms," Nanoscale Adv., vol. 2, pp. 605-625, 2020.

[54] B. Li, W. Piyawattanametha, and Z. Qiu, "Metalens-based miniaturized optical systems," Micromachines, vol. 10, p. 310, 2019.

[55] X. Ni, N. K. Emani, A. V. Kildishev, A. Boltasseva, and V. M. Shalaev, "Broadband light bending with plasmonic nanoantennas," Science (80-), vol. 335, p. 427, 2012.

[56] F. Aieta, P. Genevet, N. Yu, M. A. Kats, Z. Gaburro, and F. Capasso, "Out-of-plane reflection and refraction of light by anisotropic optical antenna metasurfaces with phase discontinuities," Nano Lett., vol. 12, pp. 1702-1706, 2012.

[57] S. Gwo, C.-Y. Wang, H.-Y. Chen, et al., "Plasmonic metasurfaces for nonlinear optics and quantitative SERS," ACS Photonics, vol. 3, pp. 1371-1384, 2016.

[58] G. Mie, "Beiträge zur Optik trüber Medien, speziell kolloidaler Metallösungen,” Ann. Phys., vol. 330, pp. 377-445, 1908.

[59] N. M. Estakhri and A. Alù, "Recent progress in gradient metasurfaces,” J. Opt. Soc. Am. B, vol. 33, p. A21, 2016. 
[60] C. Huygens, Traité de la Lumière (drafted 1678; published in Leyden by Van der Aa, 1690), translated by Silvanus P. Thompson as Treatise on Light (London: Macmillan, 1912; Project Gutenberg edition, 2005). Leyden: Van der Aa, p. 19, 1690.

[61] A. Fresnel, Mémoire sur la diffraction de la lumière" (deposited 1818, “crowned” 1819), in Oeuvres complètes (Paris: Imprimerie impériale, 1866-1870. vol.1, pp. 247-363; partly translated as “Fresnel's prize memoir on the diffraction of light”, in H. Crew (ed.), The Wave Theory of Light: Memoirs by Huygens, Young and Fresnel, American Book Co., 1900, pp. 81-144.

[62] M. V. Berry, "The adiabatic phase and pancharatnam's phase for polarized light,” J. Mod. Opt., vol. 34, pp. 1401-1407, 1987.

[63] S. Pancharatnam, "Generalized theory of interference and its applications,” Proc. Indian Acad. Sci. Sect. A, vol. 44, pp. 398-417, 1956.

[64] S. M. Kamali, A. Arbabi, E. Arbabi, Y. Horie, and A. Faraon, "Decoupling optical function and geometrical form using conformal flexible dielectric metasurfaces," Nat. Commun., vol. 7, p. 11618, 2016.

[65] S. M. Kamali, E. Arbabi, A. Arbabi, Y. Horie, and A. Faraon, "Highly tunable elastic dielectric metasurface lenses," Laser Photon. Rev., vol. 10, pp. 1002-1008, 2016.

[66] A. She, S. Zhang, S. Shian, D. R. Clarke, and F. Capasso, "Adaptive metalenses with simultaneous electrical control of focal length, astigmatism, and shift," Sci. Adv., vol. 4, p. eaap9957, 2018.

[67] H.-S. Ee and R. Agarwal, "Tunable metasurface and flat optical zoom lens on a stretchable substrate," Nano Lett., vol. 16, pp. 2818-2823, 2016.

[68] T. Tumkur, G. Zhu, P. Black, Y. A. Barnakov, C. E. Bonner, and M. A. Noginov, "Control of spontaneous emission in a volume of functionalized hyperbolic metamaterial," Appl. Phys. Lett., vol. 99, p. 151115, 2011.

[69] M. R. M. Hashemi, S.-H. Yang, T. Wang, N. Sepúlveda, and M. Jarrahi, "Electronically-controlled beam-steering through vanadium dioxide metasurfaces," Sci. Rep., vol. 6, p. 35439, 2016.

[70] D. Wang, L. Zhang, Y. Gong, et al., "Multiband switchable terahertz quarter-wave plates via phase-change metasurfaces," IEEE Photonics J, vol. 8, pp. 1-8, 2016.

[71] D. Wang, L. Zhang, Y. Gu, et al., "Switchable ultrathin quarterwave plate in terahertz using active phase-change metasurface," Sci. Rep., vol. 5, p. 15020, 2015.

[72] J. Rensberg, S. Zhang, Y. Zhou, et al., "Active optical metasurfaces based on defect-engineered phase-transition materials," Nano Lett., vol. 16, pp. 1050-1055, 2016.

[73] Q. Wang, E. T. F. Rogers, B. Gholipour, et al., “Optically reconfigurable metasurfaces and photonic devices based on phase change materials," Nat. Photonics, vol. 10, pp. 60-65, 2016.

[74] M. A. Kats, R. Blanchard, P. Genevet, et al., "Thermal tuning of mid-infrared plasmonic antenna arrays using a phase change material," Opt. Lett., vol. 38, p. 368, 2013.

[75] J. Sautter, I. Staude, M. Decker, et al., "Active tuning of alldielectric metasurfaces," ACS Nano, vol. 9, pp. 4308-4315, 2015.

[76] O. Buchnev, N. Podoliak, M. Kaczmarek, N. I. Zheludev, and V. A. Fedotov, "Electrically controlled nanostructured metasurface loaded with liquid crystal: toward multifunctional photonic switch," Adv. Opt. Mater., vol. 3, pp. 674-679, 2015.

[77] W. Zhu, Q. Song, L. Yan, et al., "A flat lens with tunable phase gradient by using random access reconfigurable metamaterial," Adv. Mater., vol. 27, pp. 4739-4743, 2015.

[78] N. Dabidian, I. Kholmanov, A. B. Khanikaev, et al., "Electrical switching of infrared light using graphene integration with plasmonic Fano resonant metasurfaces," ACS Photonics, vol. 2, pp. 216-227, 2015.

[79] Y. Yao, R. Shankar, M. A. Kats, et al., "Electrically tunable metasurface perfect absorbers for ultrathin mid-infrared optical modulators," Nano Lett., vol. 14, pp. 6526-6532, 2014.

[80] T. Roy, S. Zhang, I. W. Jung, M. Troccoli, F. Capasso, and D. Lopez, "Dynamic metasurface lens based on MEMS technology," APL Photonics, vol. 3, p. 021302, 2018.

[81] S. Zhang, A. Soibel, S. A. Keo, et al., "Solid-immersion metalenses for infrared focal plane arrays," Appl. Phys. Lett., vol. 113, p. 111104, 2018.

[82] D. Hu, X. Wang, S. Feng, et al., "Ultrathin terahertz planar elements,” Adv. Opt. Mater., vol. 1, pp. 186-191, 2013.

[83] T. Hu, Q. Zhong, N. Li, et al., "CMOS-compatible a-Si metalenses on a 12-inch glass wafer for fingerprint imaging," Nanophotonics, vol. 9, pp. 823-830, 2020.

[84] J. Lin, J. P. B. Mueller, Q. Wang, et al., "Polarization-controlled tunable directional coupling of surface plasmon polaritons," Science (80-), vol. 340, pp. 331-334, 2013.

[85] Z. Liu, H. Du, Z.-Y. Li, N. X. Fang, and J. Li, "Invited Article: nano-kirigami metasurfaces by focused-ion-beam induced close-loop transformation," APL Photonics, vol. 3, p. 100803 , 2018.

[86] M. Principe, M. Consales, A. Micco, et al., "Optical fiber metatips," Light Sci. Appl., vol. 6, p. e16226, 2017.

[87] J. Yang, I. Ghimire, P. C. Wu, et al., "Photonic crystal fiber metalens," Nanophotonics, vol. 8, pp. 443-449, 2019.

[88] G. Barbillon, "Soft UV nanoimprint lithography: a tool to design plasmonic nanobiosensors," Adv. Unconv. Lithogr., pp. 3-14, 2011. https://doi.org/10.5772/20572.

[89] Y. Yao, H. Liu, Y. Wang, et al., “Nanoimprint-defined, large-area meta-surfaces for unidirectional optical transmission with superior extinction in the visible-to-infrared range," Opt. Express, vol. 24, p. 15362, 2016.

[90] K. Kim, G. Yoon, S. Baek, J. Rho, and H. Lee, "Facile nanocasting of dielectric metasurfaces with sub-100 nm resolution," ACS Appl. Mater. Interfaces, vol. 11, pp. 26109-26115, 2019.

[91] C. A. Dirdal, G. U. Jensen, H. Angelskår, P. C. Vaagen Thrane, J. Gjessing, and D. A. Ordnung, "Towards high-throughput largearea metalens fabrication using UV-nanoimprint lithography and Bosch deep reactive ion etching," Opt. Express, vol. 28, p. 15542, 2020.

[92] H.-M. Jin, S.-J. Jeong, H.-S. Moon, et al., "Ultralarge-area block copolymer lithography using self-assembly assisted photoresist pre-pattern," in IEEE Nanotechnology Materials and Devices Conference (IEEE, 2011), 2011, pp. 527-533.

[93] J. Y. Kim, H. Kim, B. H. Kim, et al., "Highly tunable refractive index visible-light metasurface from block copolymer self-assembly," Nat. Commun., vol. 7, p. 12911, 2016.

[94] M. Mayer, M. J. Schnepf, T. A. F. König, and A. Fery, "Colloidal self-assembly concepts for plasmonic metasurfaces," Adv. Opt. Mater., vol. 7, p. 1800564, 2019. 
[95] Z. Wu, W. Li, M. N. Yogeesh, et al., "Tunable graphene metasurfaces with gradient features by self-assembly-based moiré nanosphere lithography," Adv. Opt. Mater., vol. 4, pp. 2035-2043, 2016.

[96] M. Khorasaninejad, W. T. Chen, A. Y. Zhu, et al., "Multispectral chiral imaging with a metalens," Nano Lett., vol. 16, pp. 4595-4600, 2016.

[97] N. A. Rubin, G. D’Aversa, P. Chevalier, Z. Shi, W. T. Chen, and F. Capasso, "Matrix Fourier optics enables a compact fullStokes polarization camera," Science (80-), vol. 365, p. eaax1839, 2019.

[98] H. Pahlevaninezhad, M. Khorasaninejad, Y.-W. Huang, et al., "Nano-optic endoscope for high-resolution optical coherence tomography in vivo," Nat. Photonics, vol. 12, pp. 540-547, 2018.

[99] D. Lee, M. Kim, J. Kim, et al., “All-dielectric metasurface imaging platform applicable to laser scanning microscopy with enhanced axial resolution and wavelength selection," Opt. Mater. Express, vol. 9, p. 3248, 2019.

[100] M. Jang, Y. Horie, A. Shibukawa, et al., "Wavefront shaping with disorder-engineered metasurfaces," Nat. Photonics, vol. 12, pp. 84-90, 2018.

[101] E. Arbabi, J. Li, R. J. Hutchins, et al., "Two-photon microscopy with a double-wavelength metasurface objective lens," Nano Lett., vol. 18, pp. 4943-4948, 2018.

[102] D. Sun, Y. Yang, S. Liu, et al., "Excitation and emission dualwavelength confocal metalens designed directly in the biological tissue environment for two-photon microendoscopy," Biomed. Opt. Express, vol. 11, p. 4408, 2020.

[103] J. S. Paiva, P. A. S. Jorge, C. C. Rosa, and J. P. S. Cunha, "Optical fiber tips for biological applications: from light confinement, biosensing to bioparticles manipulation," Biochim. Biophys. Acta Gen. Subj., vol. 1862, pp. 1209-1246, 2018.

[104] V. Westphal, S. O. Rizzoli, M. A. Lauterbach, D. Kamin, R. Jahn, and S. W. Hell, "Video-rate far-field optical nanoscopy dissects synaptic vesicle movement," Science (80-), vol. 320, pp. 246-249, 2008.

[105] J. M. Pullman, J. Nylk, E. C. Campbell, F. J. Gunn-Moore, M. B. Prystowsky, and K. Dholakia, "Visualization of podocyte substructure with structured illumination microscopy (SIM): a new approach to nephrotic disease," Biomed. Opt. Express, vol. 7, p. 302, 2016.

[106] F. Wei, D. Lu, H. Shen, et al., "Wide field superresolution surface imaging through plasmonic structured illumination microscopy," Nano Lett., vol. 14, pp. 4634-4639, 2014.

[107] S. Cao, T. Wang, Q. Sun, B. Hu, U. Levy, and W. Yu, “Graphene on meta-surface for super-resolution optical imaging with a sub-10 nm resolution," Opt. Express, vol. 25, p. 14494, 2017.

[108] J. L. Ponsetto, A. Bezryadina, F. Wei, et al., "Experimental demonstration of localized plasmonic structured illumination microscopy," ACS Nano, vol. 11, pp. 5344-5350, 2017.

[109] A. Bezryadina, J. Zhao, Y. Xia, X. Zhang, and Z. Liu, “High spatiotemporal resolution imaging with localized plasmonic structured illumination microscopy," ACS Nano, vol. 12, pp. 8248-8254, 2018.

[110] Q. Ma, H. Qian, S. Montoya, et al., "Experimental demonstration of hyperbolic metamaterial assisted illumination nanoscopy," ACS Nano, vol. 12, pp. 11316-11322, 2018.
[111] A. P. Slobozhanyuk, A. N. Poddubny, A. J. E. E. Raaijmakers, et al., "Enhancement of magnetic resonance imaging with metasurfaces," Adv. Mater., vol. 28, pp. 1832-1838, 2016.

[112] A. V Shchelokova, A. P. Slobozhanyuk, I. V. Melchakova, et al., "Locally enhanced image quality with tunable hybrid metasurfaces," Phys. Rev. Appl., vol. 9, p. 014020, 2018.

[113] R. Schmidt, A. Slobozhanyuk, P. Belov, and A. Webb, "Flexible and compact hybrid metasurfaces for enhanced ultra high field in vivo magnetic resonance imaging," Sci. Rep., vol. 7, p. 1678, 2017.

[114] E. A. Brui, A. V Shchelokova, M. Zubkov, I. V. Melchakova, S. B. Glybovski, and A. P. Slobozhanyuk, "Adjustable subwavelength metasurface-inspired resonator for magnetic resonance imaging," Phys. Status Solidi Appl. Mater. Sci., vol. 215, p. 1700788, 2018.

[115] A. V Shchelokova, A. P. Slobozhanyuk, P. de Bruin, et al., "Experimental investigation of a metasurface resonator for in vivo imaging at 1.5 T," J. Magn. Reson., vol. 286, pp. 78-81, 2018.

[116] Y. Park, C. Depeursinge, and G. Popescu, "Quantitative phase imaging in biomedicine," Nat. Photonics, vol. 12, pp. 578-589, 2018.

[117] H. Kwon, E. Arbabi, S. M. Kamali, M. Faraji-Dana, and A. Faraon, "Single-shot quantitative phase gradient microscopy using a system of multifunctional metasurfaces," Nat. Photonics, vol. 14, pp. 109-114, 2020.

[118] A. Jayasingh, V. Rompicherla, R. K. N. Radha, and P. Shanmugam, "Comparative study of peripheral blood smear, rapid antigen detection, ELISA and PCR methods for diagnosis of malaria in a tertiary care centre,"J. Clin. Diagn. Res., vol. 13, 2019. https:/doi. org/10.7860/JCDR/2019/38213.12483.

[119] T. Moore, A. Moody, T. Payne, G. Sarabia, A. Daniel, and B. Sharma, "In vitro and in vivo SERS biosensing for disease diagnosis,” Biosensors, vol. 8, p. 46, 2018.

[120] C. L. Wong and M. Olivo, "Surface plasmon resonance imaging sensors: a review,” Plasmonics, vol. 9, pp. 809-824, 2014.

[121] V. Bochenkov and T. Shabatina, "Chiral plasmonic biosensors," Biosensors, vol. 8, p. 120, 2018.

[122] J. R. Mejía-Salazar and O. N. Oliveira, "Plasmonic biosensing," Chem. Rev., vol. 118, pp. 10617-10625, 2018.

[123] Y. Esfahani Monfared, "Overview of recent advances in the design of plasmonic fiber-optic biosensors," Biosensors, vol. 10, p. 77, 2020.

[124] A. K. Sharma and C. Marques, "Design and performance perspectives on fiber optic sensors with plasmonic nanostructures and gratings: a review," IEEE Sens. J., vol. 19, pp. 7168-7178, 2019.

[125] X. Yu, S. Zhang, M. Olivo, and N. Li, “Micro- and nano-fiber probes for optical sensing, imaging, and stimulation in biomedical applications," Photonics Res. Early Post, 2020. https://doi.org/10.1364/prj.387076.

[126] A. Salim and S. Lim, "Review of recent metamaterial microfluidic sensors," Sensors, vol. 18, p. 232, 2018.

[127] A. V. Kabashin, P. Evans, S. Pastkovsky, et al., "Plasmonic nanorod metamaterials for biosensing," Nat. Mater., vol. 8 , pp. 867-871, 2009.

[128] R. Singh, W. Cao, I. Al-Naib, L. Cong, W. Withayachumnankul, and W. Zhang, "Ultrasensitive terahertz sensing with high-Q Fano resonances in metasurfaces," Appl. Phys. Lett., vol. 105, p. 171101, 2014.

[129] Z. Li, Y. Zhu, Y. Hao, et al., "Hybrid metasurface-based midinfrared biosensor for simultaneous quantification and 
identification of monolayer protein," ACS Photonics, vol. 6, pp. 501-509, 2019.

[130] Y. Zhu, Z. Li, Z. Hao, et al., “Optical conductivity-based ultrasensitive mid-infrared biosensing on a hybrid metasurface," Light Sci. Appl., vol. 7, pp. 1-11, 2018.

[131] F. Yesilkoy, E. R. Arvelo, Y. Jahani, et al., "Ultrasensitive hyperspectral imaging and biodetection enabled by dielectric metasurfaces," Nat. Photonics, vol. 13, pp. 390-396, 2019.

[132] A. Tittl, A. Leitis, M. Liu, et al., “Imaging-based molecular barcoding with pixelated dielectric metasurfaces," Science (80-), vol. 360, pp. 1105-1109, 2018.

[133] R. H. Siddique, S. Kumar, V. Narasimhan, H. Kwon, and H. Choo, "Aluminum metasurface with hybrid multipolar plasmons for 1000-fold broadband visible fluorescence enhancement and multiplexed biosensing," ACS Nano, vol. 13, pp. 13775-13783, 2019.

[134] R. Ahmed, M. O. Ozen, M. G. Karaaslan, et al., "Tunable fanoresonant metasurfaces on a disposable plastic-template for multimodal and multiplex biosensing," Adv. Mater., vol. 32, p. 1907160, 2020.

[135] Q. Guo, H. Zhu, F. Liu, et al., "Silicon-on-Glass graphenefunctionalized leaky cavity mode nanophotonic biosensor," ACS Photonics, vol. 1, pp. 221-227, 2014.

[136] L. Jiang, S. Zeng, Z. Xu, et al., "Multifunctional hyperbolic nanogroove metasurface for submolecular detection," Small, vol. 13, p. 1700600, 2017.

[137] C. Jack, A. S. Karimullah, R. Leyman, et al., "Biomacromolecular stereostructure mediates mode hybridization in chiral plasmonic nanostructures," Nano Lett., vol.16, pp. 5806-5814, 2016.

[138] A. Leitis, A. Tittl, M. Liu, et al., “Angle-multiplexed all-dielectric metasurfaces for broadband molecular fingerprint retrieval," Sci. Adv., vol. 5, p. eaaw2871, 2019.

[139] W. Xu, L. Xie, J. Zhu, et al., "Terahertz biosensing with a graphene-metamaterial heterostructure platform," Carbon $N$. Y., vol. 141, pp. 247-252, 2019.

[140] H.-K. Lee, S. J. Kim, Y. H. Kim, Y. Ko, S. Ji, and J.-C. Park, "Odontogenic ameloblast-associated protein (ODAM) in gingival crevicular fluid for site-specific diagnostic value of periodontitis: a pilot study," BMC Oral Health, vol. 18, p. 148, 2018.

[141] O. Limaj, D. Etezadi, N. J. Wittenberg, et al., “Infrared plasmonic biosensor for real-time and label-free monitoring of lipid membranes," Nano Lett., vol. 16, pp. 1502-1508, 2016.

[142] N. Muhammad, Q. Liu, X. Tang, T. Fu, A. Daud Khan, and Z. Ouyang, "Highly flexible and voltage based wavelength tunable biosensor," Phys. Status Solidi, vol. 216, p. 1800633 , 2019.

[143] S. J. Park, J. T. Hong, S. J. Choi, et al., "Detection of microorganisms using terahertz metamaterials," Sci. Rep., vol. 4, p. 4988, 2015.

[144] C. Zhang, L. Liang, L. Ding, et al., "Label-free measurements on cell apoptosis using a terahertz metamaterial-based biosensor," Appl. Phys. Lett., vol. 108, p. 241105, 2016.

[145] D. Rodrigo, A. Tittl, N. Ait-Bouziad, et al., "Resolving moleculespecific information in dynamic lipid membrane processes with multi-resonant infrared metasurfaces," Nat. Commun., vol. 9, p. $2160,2018$.
[146] Y. Wang, M. A. Ali, E. K. C. Chow, L. Dong, and M. Lu, “An optofluidic metasurface for lateral flow-through detection of breast cancer biomarker," Biosens. Bioelectron., vol. 107, pp. 224-229, 2018

[147] Z. Geng, X. Zhang, Z. Fan, X. Lv, and H. Chen, “A route to terahertz metamaterial biosensor integrated with microfluidics for liver cancer biomarker testing in early stage," Sci. Rep., vol. 7, p. 16378, 2017.

[148] X. Yan, M. Yang, Z. Zhang, et al., "The terahertz electromagnetically induced transparency-like metamaterials for sensitive biosensors in the detection of cancer cells," Biosens. Bioelectron., vol. 126, pp. 485-492, 2019.

[149] D. Vrba, J. Vrba, D. B. Rodrigues, and P. Stauffer, "Numerical investigation of novel microwave applicators based on zero-order mode resonance for hyperthermia treatment of cancer," J. Franklin Inst., vol. 354 pp. 8734-8746, 2017.

[150] J. N. Anker, W. P. Hall, O. Lyandres, N. C. Shah, J. Zhao, and R. P. Van Duyne, "Biosensing with plasmonic nanosensors," in Nanoscience and Technology, UK, Co-Published with Macmillan Publishers Ltd, 2009, pp. 308-319.

[151] K. A. Willets and R. P. Van Duyne, "Localized surface plasmon resonance spectroscopy and sensing," Annu. Rev. Phys. Chem., vol. 58, pp. 267-297, 2007.

[152] P. L. Stiles, J. A. Dieringer, N. C. Shah, and R. P. Van Duyne, "Surface-enhanced Raman spectroscopy," Annu. Rev. Anal. Chem., vol. 1, pp. 601-626, 2008.

[153] C. L. Wong, J. Y. Chan, L. X. Choo, H. Q. Lim, H. Mittman, and M. Olivo, "Plasmonic contrast imaging biosensor for the detection of H3N2 influenza protein-antibody and DNA-DNA molecular binding," IEEE Sens. J., vol. 19, pp. 11828-11833, 2019.

[154] C. L. Wong, M. Chua, H. Mittman, L. X. Choo, H. Q. Lim, and M. Olivo, "A phase-intensity surface plasmon resonance biosensor for avian influenza A (H5N1) detection," Sensors, vol. 17, p. 2363, 2017.

[155] C. L. Wong, U. S. Dinish, K. D. Buddharaju, M. S. Schmidt, and M. Olivo, "Surface-enhanced Raman scattering (SERS)-based volatile organic compounds (VOCs) detection using plasmonic bimetallic nanogap substrate," Appl. Phys. A, vol. 117, pp. 687-692, 2014.

[156] C. L. Wong, G. C. K. Chen, X. Li, et al., "Colorimetric surface plasmon resonance imaging (SPRI) biosensor array based on polarization orientation," Biosens. Bioelectron., vol. 47, pp. 545-552, 2013.

[157] C. L. Wong, H. P. Ho, K. S. Chan, and S. Y. Wu, "Application of surface plasmon resonance sensing to studying elastohydrodynamic lubricant films," Appl. Opt., vol. 44, p. 4830, 2005.

[158] C. L. Wong, H. P. Ho, K. S. Chan, P. L. Wong, S. Y. Wu, and C. Lin, "Optical characterization of elastohydrodynamic lubricated (EHL) contacts using surface plasmon resonance (SPR) effect," Tribol. Int., vol. 41, pp. 356-366, 2008.

[159] W.-I. K. Chio, W. J. Peveler, K. I. Assaf, et al., "Selective detection of nitroexplosives using molecular recognition within self-assembled plasmonic nanojunctions," J. Phys. Chem. C, vol. 123, pp. 15769-15776, 2019.

[160] Y. Lee, S.-J. Kim, H. Park, and B. Lee, "Metamaterials and metasurfaces for sensor applications," Sensors, vol. 17, p. 1726, 2017. 
[161] S. L. Dodson, C. Cao, H. Zaribafzadeh, S. Li, and Q. Xiong, "Engineering plasmonic nanorod arrays for colon cancer marker detection," Biosens. Bioelectron., vol. 63, pp. 472-477, 2015.

[162] S. Chen, M. Svedendahl, M. Käll, L. Gunnarsson, and A. Dmitriev, "Ultrahigh sensitivity made simple: nanoplasmonic label-free biosensing with an extremely low limit-of-detection for bacterial and cancer diagnostics," Nanotechnology, vol. 20, p. 434015, 2009.

[163] J. Yuan, Duan, Luo Yang, and M. Xi, “Detection of serum human epididymis secretory protein 4 in patients with ovarian cancer using a label-free biosensor based on localized surface plasmon resonance," Int. J. Nanomed., vol. 7, pp. 2921-2928, 2012.

[164] E. Ouellet, C. Lausted, T. Lin, C. W. T. Yang, L. Hood, and E. T. Lagally, "Parallel microfluidic surface plasmon resonance imaging arrays," Lab Chip, vol. 10, p. 581, 2010.

[165] D. Kim and A. E. Herr, "Protein immobilization techniques for microfluidic assays," Biomicrofluidics, vol. 7, p. 041501, 2013.

[166] H. Aouani, M. Rahmani, H. Šípová, et al., "Plasmonic nanoantennas for multispectral surface-enhanced spectroscopies,"J. Phys. Chem. C, vol. 117, pp. 18620-18626, 2013.

[167] S. Zeng, K. V. Sreekanth, J. Shang, et al., “Graphene-gold metasurface architectures for ultrasensitive plasmonic biosensing," Adv. Mater., vol. 27, pp. 6163-6169, 2015

[168] S. Zeng, S. Hu, J. Xia, et al., "Graphene-MoS2 hybrid nanostructures enhanced surface plasmon resonance biosensors," Sens. Actuators B Chem., vol. 207, pp. 801-810, 2015.

[169] M. Booth, D. Andrade, D. Burke, B. Patton, and M. Zurauskas, "Aberrations and adaptive optics in super-resolution microscopy," Microscopy, vol. 64, pp. 251-261, 2015.

[170] N. Ji, “Adaptive optical fluorescence microscopy," Nat. Methods, vol. 14, pp. 374-380, 2017.

[171] M. J. Booth, M. A. A. A. Neil, R. Juskaitis, and T. Wilson, "Adaptive aberration correction in a confocal microscope," Proc. Natl. Acad. Sci., vol. 99, pp. 5788-5792, 2002.

[172] E. Arbabi, A. Arbabi, S. M. Kamali, Y. Horie, M. Faraji-Dana, and A. Faraon, "MEMS-tunable dielectric metasurface lens," Nat. Commun., vol. 9, p. 812, 2018.

[173] P. Vaiano, B. Carotenuto, M. Pisco, et al., "Lab on fiber technology for biological sensing applications," Laser Photon. Rev., vol. 10, pp. 922-961, 2016.

[174] E. J. Smythe, M. D. Dickey, J. Bao, G. M. Whitesides, and F. Capasso, "Optical antenna arrays on a fiber facet for in situ surface-enhanced Raman scattering detection," Nano Lett., vol. 9, pp. 1132-1138, 2009.

[175] Y.-W. Huang, N. A. Rubin, A. Ambrosio, et al., "Versatile total angular momentum generation using cascaded J-plates," Opt. Express, vol. 27, p. 7469, 2019.

[176] R. C. Devlin, A. Ambrosio, N. A. Rubin, J. P. B. Mueller, and F. Capasso, "Arbitrary spin-to-orbital angular momentum conversion of light," Science (80-), vol. 358, pp. 896-901, 2017.

[177] W. T. Chen, M. Khorasaninejad, A. Y. Zhu, et al., "Generation of wavelength-independent subwavelength Bessel beams using metasurfaces," Light Sci. Appl., vol. 6, p. e16259, 2017.

[178] S. Kang, H. E. Joe, J. Kim, Y. Jeong, B. K. Min, and K. Oh, "Subwavelength plasmonic lens patterned on a composite optical fiber facet for quasi-one-dimensional Bessel beam generation," Appl. Phys. Lett., vol. 98, pp. 1-4, 2011.

[179] W. Yu, Z. Ji, D. Dong, et al., "Super-resolution deep imaging with hollow Bessel beam STED microscopy," Laser Photon. Rev., vol. 10, pp. 147-152, 2016.

[180] T. Meinert and A. Rohrbach, "Light-sheet microscopy with length-adaptive Bessel beams," Biomed. Opt. Express, vol. 10, p. 670, 2019.

[181] R. Lu, W. Sun, Y. Liang, et al., "Video-rate volumetric functional imaging of the brain at synaptic resolution," Nat. Neurosci., vol. 20, pp. 620-628, 2017.

[182] K. Wu, P. Coquet, Q. J. Wang, and P. Genevet, "Modelling of freeform conformal metasurfaces," Nat. Commun., vol. 9, p. 3494, 2018.

[183] K. Liu, T. Tachi, and G. H. Paulino, "Invariant and smooth limit of discrete geometry folded from bistable origami leading to multistable metasurfaces," Nat. Commun., vol. 10, p. 4238, 2019.

[184] G. Tkachenko, D. Stellinga, A. Ruskuc, M. Chen, K. Dholakia, and T. F. Krauss, "Optical trapping with planar silicon metalenses," Opt. Lett., vol. 43, p. 3224, 2018.

[185] S. Yi, M. Zhou, Z. Yu, et al., "Subwavelength anglesensing photodetectors inspired by directional hearing in small animals," Nat. Nanotechnol., vol. 13, pp. 1143-1147, 2018.

[186] N. N. Shi, C. C. Tsai, F. Camino, G. D. Bernard, N. Yu, and R. Wehner, "Keeping cool: enhanced optical reflection and radiative heat dissipation in Saharan silver ants," Science (80-), vol. 349, pp. 298-301, 2015.

[187] A. Basiri, X. Chen, J. Bai, et al., "Nature-inspired chiral metasurfaces for circular polarization detection and full-Stokes polarimetric measurements," Light Sci. Appl., vol. 8, p. 78, 2019.

[188] Q. Guo, Z. Shi, Y.-W. Huang, et al., "Compact single-shot metalens depth sensors inspired by eyes of jumping spiders," Proc. Natl. Acad. Sci. USA, vol. 116, pp. 22959-22965, 2019.

[189] Z.-B. Fan, Z.-K. Shao, M.-Y. Xie, et al., "Silicon nitride metalenses for close-to-one numerical aperture and wide-angle visible imaging," Phys. Rev. Appl., vol. 10, p. 014005, 2018.

[190] S. Wang, P. C. Wu, V.-C. Su, et al., "A broadband achromatic metalens in the visible," Nat. Nanotechnol., vol. 13, pp. 227-232, 2018

[191] W. T. Chen, A. Y. Zhu, V. Sanjeev, et al., "A broadband achromatic metalens for focusing and imaging in the visible," Nat. Nanotechnol., vol. 13, pp. 220-226, 2018.

[192] A. Arbabi, E. Arbabi, S. M. Kamali, Y. Horie, S. Han, and A. Faraon, "Miniature optical planar camera based on a wide-angle metasurface doublet corrected for monochromatic aberrations," Nat. Commun., vol. 7, p. 13682, 2016.

[193] Z. Lin, B. Groever, F. Capasso, A. W. Rodriguez, and M. Lončar, "Topology-optimized multilayered metaoptics," Phys. Rev. Appl., vol. 9, p. 044030, 2018.

[194] B. C. Wilson, M. Jermyn, and F. Leblond, "Challenges and opportunities in clinical translation of biomedical optical spectroscopy and imaging," J. Biomed. Opt., vol. 23, p. 1, 2018.

[195] J. Popp, D. Matthews, A. Martinez-Coll, T. Mayerhöfer, and B. C. Wilson, "Challenges in translation: models to 
promote translation," J. Biomed. Opt., vol. 23,

p. 1, 2017.

[196] B. Spackova, P. Wrobel, M. Bockova, and J. Homola, "Optical biosensors based on plasmonic nanostructures: a review," Proc. IEEE, vol. 104, pp. 2380-2408, 2016.

[197] M. A. Otte, B. Sepúlveda, W. Ni, J. P. Juste, L. M. Liz-Marzán, and L. M. Lechuga, "Identification of the optimal spectral region for plasmonic and nanoplasmonic sensing," ACS Nano, vol. 4, pp. 349-357, 2010.

[198] J. Homola, Ed., Surface Plasmon Resonance Based Sensors, Springer Series on Chemical Sensors and Biosensors, vol. 4, Springer Berlin Heidelberg, 2006.
[199] F. J. Rodríguez-Fortuño, M. Martínez-Marco, B. Tomás-Navarro, et al., "Highly-sensitive chemical detection in the infrared regime using plasmonic gold nanocrosses," Appl. Phys. Lett., vol. 98, p. 133118, 2011.

[200] S. K. Dondapati, T. K. Sau, C. Hrelescu, T. A. Klar, F. D. Stefani, and J. Feldmann, "Label-free biosensing based on single gold nanostars as plasmonic transducers," ACS Nano, vol. 4, pp. 6318-6322, 2010.

[201] P. Kvasnička and J. Homola, "Optical sensors based on spectroscopy of localized surface plasmons on metallic nanoparticles: sensitivity considerations," Biointerphases, vol. 3, pp. FD4-FD11, 2008. 